\title{
Reinforcement Learning for Reducing Congestion in Mixed Autonomy Highways
}

\author{
By \\ Aditya Maheshwari \\ A thesis proposal submitted to \\ the Faculty of Graduate and Postdoctoral Affairs \\ in partial fulfilment of \\ the requirements for the degree of \\ Master of Computer Science \\ Ottawa-Carleton Institute for Computer Science \\ School of Computer Science \\ Carleton University \\ Ottawa, Ontario
}

August 2020

(C) Copyright

2020, Aditya Maheshwari 
The undersigned hereby recommend to the Faculty of Graduate and Postdoctoral Affairs acceptance of the thesis,

\section{Reinforcement Learning for Reducing Congestion in Mixed}

\section{Autonomy Highways}

submitted by

Aditya Maheshwari

Dr. Michel Barbeau
(Director, School of Computer Science)

Dr. B. John Oommen

(Thesis Supervisor)

Carleton University

August 2020 


\section{Abstract}

Highway traffic dynamics are extremely complicated, due to the erratic behaviour of human drivers. Overreacting to avoid collisions, often by suddenly decelerating, causes waves of cars behind a driver to also slow down or stop, resulting in an emergent traffic jam. This results in many cars on the road travelling at a speed much lower than the theoretical maximum average speed of the road, and these delays create a significant cost to the economy. Inefficient driving behaviours have led to the increased interest in Autonomous Vehicles (AVs). There has also been a large amount of research on applying Machine Learning, and specifically Reinforcement Learning (RL), by using large amounts of data collected from the road, to ensure that individual AVs remain collision-free and more fuel efficient, and to maximize flow on roads that exclusively contain AVs. However, very little research has been done on mixed autonomy roads, especially in terms of improving traffic flow with AVs that are coordinated, but trained using their local observations of the road.

This thesis focuses on assessing the impact of AVs on improving traffic flow in mixed autonomy collision-free highways. Roads were simulated using micro-traffic models, with adjustments designed to simulate a future world in which Automatic Braking Systems will be good enough to completely prevent rear-end and lane-change collisions on highways. We combined Deep Neural Networks with Reinforcement Learning (Deep Q Networks) to model the AVs, and this approach was tested on single and double lane highways. Among many other salient issues, our results show that replacing only $5 \%$ of human-drivers with AVs can, remarkably, improve average road speeds by up to $10 \%$ on single lane roads, up to $24 \%$ on double lane roads, and also significantly reduce the percentage of fully-stopped cars on average, for most situations. Furthermore, these trends, for the most part, continue to amplify as higher percentages of the human drivers are replaced with AVs. 


\section{Acknowledgements}

First and foremost, I would like to acknowledge and thank my Supervisor, Prof B. John Oommen, for his support and guidance throughout the process of writing this thesis. His course, "Learning Systems for Random Environments", provided the spark that inspired me to pursue Reinforcement Learning, which was the major component of this thesis. Making the extra effort to adapt the thesis guidance from in-person to online, during the COVID-19 pandemic, involved jumping over a few extra hurdles to ensure that the work maintained its quality. Furthermore, continuously making the time to edit and guide my work, while also exercising a lot of patience in allowing me to navigate some of the uncertainty of exploring a very new field, was greatly appreciated.

I would also like to offer a huge thanks to the Computer Science Faculty at Carleton. All of the courses I took contributed to skills used in this work. Specifically, the course I took on "Evolving Information Networks", with Prof. Evangelos Kranakis, had a term project that allowed me to explore micro-traffic models, which also inspired and led to the initial study of the traffic component of this thesis. Additionally, I would like to thank the committee members, Dr. Majid Komeili and Dr. Patrick Boily, for providing detailed and insightful feedback on my work.

It is also hard to overstate how helpful and important it was that many notable researchers in the field of Reinforcement Learning have allowed their lectures and textbooks to be accessed for free, for anyone, online. In particular, the lectures online by David Silver, professor and lead researcher of the AlphaGo project, by Prof. Emma Brunskill at Stanford, and the free textbook, "Reinforcement Learning", written by Sutton and Barto, were all fantastic resources that helped me understand this new, complicated, and quickly growing field.

Finally, I would like to sincerely thank my support circle, including my friends, extended family, and especially, my parents and younger brother. 


\section{Contents}

1 Introduction 1

1.1 Introduction . . . . . . . . . . . . . . . . . . . . . 1

1.2 Motivation of the Thesis . . . . . . . . . . . . . . 3

1.3 Objectives of the Thesis . . . . . . . . . . . . . 5

1.4 Contributions of the Thesis . . . . . . . . . . . . . 6

1.5 Organization of the Thesis . . . . . . . . . . . . . . . . . 7

2 Literature Review $\quad 10$

2.1 Introduction . . . . . . . . . . . . . . . . . . . . 10

2.2 Micro Traffic Modelling . . . . . . . . . . . . . . . . . . 11

2.2.1 NS Traffic Modelling . . . . . . . . . . . . . . . . . . 12

2.2 .2 IDM Traffic Modelling . . . . . . . . . . . . . . . . . . . 16

2.3 Reinforcement Learning . . . . . . . . . . . . . . . . . . . . . . . 17

2.3.1 The Reinforcement Learning Problem . . . . . . . . . . . . . . 17

2.3.2 Markov Decision Processes . . . . . . . . . . . . . . . . . . . . 19

2.3.3 Temporal Difference Learning . . . . . . . . . . . . . . . 20

2.3.4 Deep Q Learning . . . . . . . . . . . . . . . 25

2.4 Modelling Autonomous Vehicles . . . . . . . . . . . . . . . . . 31

2.4.1 Mixed-Autonomy Road Traffic . . . . . . . . . . . . . . . . . . 32

2.4.2 AVs and Mitigating Congestion . . . . . . . . . . . . . . 35

2.4.3 Single AVs Navigating Roads Safely and Efficiently . . . . . . 38

2.5 Conclusions . . . . . . . . . . . . . . . . . . . 42 
3 Traffic Representations $\quad 44$

3.1 Introduction . . . . . . . . . . . . . . . . . . . . . . . 44

3.2 Traffic Representations . . . . . . . . . . . . . . . . . . 45

3.2.1 Road Representation ................ 45

3.2 .2 Car Representation . . . . . . . . . . . . . . . 47

3.2.3 Characteristics of Traffic Environments . . . . . . . . . . 48

3.3 Road Updates . . . . . . . . . . . . . . . . . . . . . . . . 49

3.3.1 New Car Entry . . . . . . . . . . . . . . . 50

3.3.2 Road Gap Updating Algorithm . . . . . . . . . . . . . 50

3.3 .3 Exiting Cars . . . . . . . . . . . . . . . 52

3.4 Experiment Assessment Criteria . . . . . . . . . . . . . . . . 54

3.5 Conclusions . . . . . . . . . . . . . . . . 55

4 Stochastic Traffic Patterns $\quad 56$

4.1 Introduction . . . . . . . . . . . . . . . 56

4.2 Car Updating . . . . . . . . . . . . . . . . . . 57

4.2 .1 Lane Decisions . . . . . . . . . . . . . . 57

4.2 .2 Speed Decisions . . . . . . . . . . . . . . . . 62

4.3 Experimental Results . . . . . . . . . . . . . . . . 64

4.3.1 Single-Lane Results . . . . . . . . . . . . . . . . 66

4.3 .2 Double-Lane Results . . . . . . . . . . . . . . . . . 69

4.3.3 Extensions to More Road Settings . . . . . . . . . . . . . 73

4.4 Main Results . . . . . . . . . . . . . . . . . . . . 74

4.5 Conclusions .......................... 75

5 Mixed-Autonomy Traffic Patterns $\quad 77$

5.1 Introduction . . . . . . . . . . . . . . . . . 77

5.2 Defining the RL Environment . . . . . . . . . . . . . 78

5.3 Single Lane Experiments . . . . . . . . . . . . . . . . . . . . . . . . 79

5.3.1 Tabular Reinforcement Learning . . . . . . . . . . . . . . 79

5.3.2 Approximate Reinforcement Learning Methods . . . . . . . . 83

5.3.3 Deep Q Networks . . . . . . . . . . . . . . 85 
5.4 Single Lane AVs in Double Lane Experiments . . . . . . . . . . . . 90

5.4.1 State Space and Network Architecture . . . . . . . . . . 90

5.4 .2 Results and Analysis . . . . . . . . . . . . . . . . 91

5.5 AVs and Lane Changing . . . . . . . . . . . . . . . . . . 94

5.5.1 State Space and Network Architecture . . . . . . . . . . 95

5.5.2 Results and Analysis . . . . . . . . . . . . . . . 95

5.6 Main Results . . . . . . . . . . . . . . . . . . . . . . . . . 97

5.7 Conclusions . . . . . . . . . . . . . . . . . . . . 100

6 Conclusions and Future Research 102

6.1 Summary . . . . . . . . . . . . . . . . . . . . . . . 102

6.2 Future Work . . . . . . . . . . . . . . . . . . . . . . . . 104

$\begin{array}{ll}\text { Bibliography } & 106\end{array}$

$\begin{array}{ll}\text { Appendices } & 109\end{array}$

$\begin{array}{ll}\text { A Appendix A } & 110\end{array}$

A.1 Technical Specifications . . . . . . . . . . . . . . . . . . . 110

A.2 Results Tables . . . . . . . . . . . . . . . . . . . . 111

B Appendix B 116

B.1 $n$-Lane Simulation Results . . . . . . . . . . . . . . . . . . . 116

B.2 Experiments Involving Changing Densities . . . . . . . . . . . . 119

B.2.1 Changing Density Targets by a Constant Number of Cars . . . 121

B.2.2 Changing Density Targets by a Proportionally by Density . . 121

B.3 Conclusions . . . . . . . . . . . . . . . . . . . . 125 


\section{List of Figures}

3.1 Example of a 3-lane road. . . . . . . . . . . . . . . . . . 45

4.1 An example of a 3-Lane Road, where coloured regions represent the available cells for each vehicle. Notice that no two vehicles are able to travel to the same cell, and therefore a collision will not occur, due to the lane-change and speed constraints. . . . . . . . . . . . .

4.2 The Speed Graph (SG) comparing the experiments for sparse single lane roads with a 0.1 density (left), and dense single lane roads with a 0.2 density (right), both for all four car types. . . . . . . . . . .

4.3 The Dynamics-Graph (DG) for the sparse (left) and dense (right) single lane road experiment consisting of mixed car types, represented by the dark red line in Figure $4.2 \ldots \ldots \ldots \ldots$. . . . . . . . .

4.4 The Speed Graph (SG) comparing experiments for sparse single lane roads with a 0.1 density (left), and dense single lane roads with a 0.2 density (right), where all experiments involved perturbing the road every 30 iterations on every 100 cells. . . . . . . . . . . . . .

4.5 The Speed Graph (SG) comparing sparse double lane roads consisting of a 0.1 density (left), and dense double lane roads consisting of 0.2 (right). Note that twice as many cars are needed in a double lane road to reach the same density as a single lane road, because density is proportionate to the total number of road cells and not the road length. 70 
4.6 The speed graph (SG) for closed roads (left), and open roads (right) for four experiments with $0.05,0.1,0.15$, and 0.2 densities, all of mixed types. The open roads consisted of 5 exits and entries, with an exit-rate of 0.005 , meaning each car had an expected exit time of 200 iterations. Furthermore, for both open and closed roads, cars were randomly perturbed, similar to the single lane scenarios. . . . . . . . . . . 71

4.7 The change-graph for all four closed road double lane experiments, with speed on the left and the number of lane changes on the right. .

4.8 The change-graph for all four open road double lane experiments, with speed on the left and the number of lane changes on the right. . . . .

4.9 Left: The dynamics graph for the 0.2 density closed road double lane experiment (left) and open road double lane experiment (right). . . .

5.1 The Average Speed of all cars on the road (left) and percentage of cars that are stopped (right) during the exploration phase of tabular single lane RL experiments, including the SV convergence value (horizontal black line). . . . . . . . . . . . . . . .

5.2 The Average Speed of all cars on the road (left) and percentage of cars that are stopped (right) once the table values converged for tabular single lane RL experiments, including the SV convergence value (horizontal black line). . . . . . . . . . . . . . . . .

5.3 The exploration phase (left) and convergence (right) Average Speeds for Linear-Regression Based Single Lane RL experiments, including the SV convergence value (horizontal black line). . . . . . . . . . . .

5.4 The exploration phase (left) and convergence (right) Average Speeds for XGBooost Based Single Lane RL experiments, excluding RF5, because the results were poor (included average speeds under 2.5). . . .

5.5 The exploration phase (left) and convergence (right) Average Speeds for Deep Q-Network Based Single Lane RL experiments, trained with a smaller NN, including the SV convergence value (horizontal black line). 87 
5.6 The exploration phase (left) and convergence (right) Average Speeds for Deep Q-Network Based Single Lane RL experiments, trained with a larger NN, including the SV convergence value (horizontal black line). 87

5.7 The exploration phase (left) and convergence (right) Average Percent of Stopped Cars for Deep Q-Network Based Single Lane Experiments, trained with a larger NN, including the SV convergence value (horizontal black line). . . . . . . . . . . . . . . . . . 88

5.8 The exploration phase, or starting (left) and convergence, or finishing (right) Mean Absolute Change in Speed per Iteration for Deep QNetwork Based Single Lane Experiments, trained with a larger NN, including the SV convergence value (horizontal black line). . . . . . .

5.9 Starting and finishing stopping percentages for NN-based single lane RL experiments on sparse and dense double lane roads, including the convergence values of the fully SV experiments (red for sparse roads, and black for dense roads). . . . . . . . . . . . .

5.10 Starting and finishing stopping percentages for NN-based single lane $\mathrm{RL}$ experiments on sparse and dense double lane roads, including the convergence values of the fully SV experiments (red for sparse roads, and black for dense roads). . . . . . . . . . . . . . . . 92

5.11 Convergence average speeds for NN-based single lane RL experiments on sparse and dense double lane open roads, including convergence values for both closed and open roads (grey/black for dense roads, and brown $/$ red for sparse roads). . . . . . . . . . . . . . . . 93

5.12 Starting and finishing stopping percentages for NN-based double lane RL experiments on double lane roads. . . . . . . . . . . . . 95

5.13 Starting and finishing stopping percentages for NN-based double lane RL experiments on double lane roads. . . . . . . . . . . . . 96

5.14 Finishing Average speed for NN-based double lane RL experiments on double lane open roads. . . . . . . . . . . . . . . 97

A.1 Closed Road SV Results for Different Car Types. . . . . . . . . . 112 
A.2 Single Lane Results with AVs. . . . . . . . . . . . . . . . . . 113

A.3 Double Lane Results With Single Lane AVs. . . . . . . . . . . . . . 114

A.4 Double Lane Results With Double Lane AVs. . . . . . . . . . . . . 115

B.1 Speed Graphs for Sparse (left) and Dense (right) 3-lane and 4-lane Open Road Experiments, including one forced slowdown to the maximum speed of 2 for 25 road cells at the beginning of the first lane, and also with random perturbations. . . . . . . . . . . . . 117

B.2 Dynamics-Graph for the 3 lane (left) and 4 lane (right) Sparse Open Road Experiments . . . . . . . . . . . . . . . . 118

B.3 Dynamics-Graph for the 3 lane (left) and 4 lane (right) Dense Open Road Experiments . . . . . . . . . . . . . . . . 118

B.4 Change-graph for the 3 lane and 4 lane Dense Open Road Experiments 119

B.5 Comparison of constant density changes for 1 lane, 2 lane, 3 lane, and 4 lane roads. All experiments started with 50 cars, and after every 60 iterations, the density target was increased by 50 cars for 10 iterations, and then decreased by 50 cars again for 10 iterations, finishing with the final 10 iterations again at a target of 50 cars. The speed graph is given on the left, and graph plotting the number of cars is on the right. 120

B.6 The change-graph for the constant density changes across multiple lanes.120

B.7 Comparison of proportional density changes for 1 lane, 2 lane, 3 lane, and 4 lane roads. All experiments started with a density of 0.05 , and on every 60 iterations, the density target was increased by 0.05 for 10 iterations, and then decreased by 0.05 again for 10 iterations, finishing with the final 10 iterations again at the initial density of 0.05 . The speed graph is given on the left, and graph plotting the number of cars

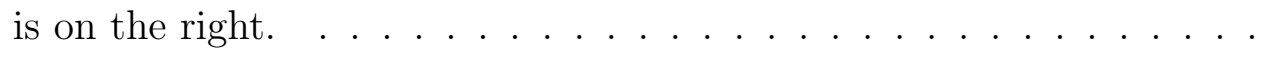

B.8 The change-graph for the proportional density changes across multiple lanes. . . . . . . . . . . . . . . . . . . . . .

B.9 The dynamics-graph for the 1 lane (left) and 2 lane (right) proportional density change experiments. . . . . . . . . . . . . . 123 
B.10 The dynamics-graph for the 3 lane (left) and 4 lane (right) proportional density change experiments. . . . . . . . . . . . . . . 123 


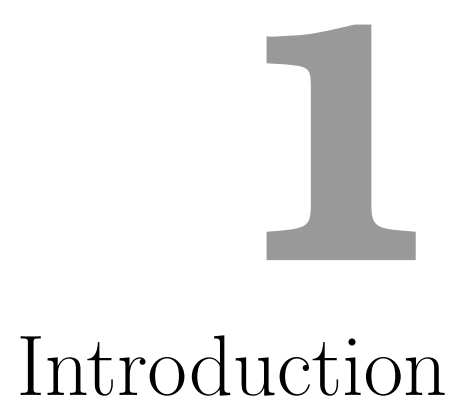

\section{$1.1 \quad$ Introduction}

Safe, reliable, and efficient movement of people and goods plays a critical role in maintaining the modern consumption-heavy lifestyle that exists today. According to the United States Department of Transportation, as of 2017, trucks accounted for $71 \%$ of all freight in America, and cars accounted for $87 \%$ of all daily trips, which emphasizes how essential efficient roads are in enabling supply chains [3]. In fact, any delays on roadways come with a significant economic cost! A study released by the Texas A\&M Transportation Institute revealed that traffic costed the US economy a total of 179 billion dollars per year, and the average commuter spent 54 hours per year stuck in traffic, valued at $\$ 18.12$ per hour for personal travel and at $\$ 52.14$ per hour for commercial travel [3]. Similarly, according to the Organization for Economic Co-operation and Development, a commuter spends 47 hours over 240 commuting 
days in a year on average, to and from work, in Toronto, Canada. This costs each household roughly $\$ 125$ per year, as well as, in total for the city, 3.3 billion dollars in lost productivity and 2.7 billion dollars in lost opportunities for growth [13].

Traffic occurs predominantly as a result of inefficient driving behaviours that result in collisions and emergent traffic jams. According to statistics from the Canadian Automobile Association (CAA), collisions are 94\% due to human error, and $65 \%$ due to driver inattention. Distracted driving and the resulting miscommunications are well known to have devastating consequences, but nevertheless, $47 \%$ of the drivers surveyed by the CAA admitted to continue partaking in such behaviours. Fundamentally, this because the human brain does not excel at tasks which are repetitive and "boring", and also tends to overreact when dealing with suddenly stressful situations (known as the "fight-or-flight" response). Driving is one of the unique activities which falls into both categories: generally boring, with the possibility of becoming suddenly stressful. Therefore, it is likely that collisions will only be completely eliminated when the techniques which are used for Automatic Braking Sensors (ABS) and cruise control are perfected.

Avoiding collisions is not enough to allow for roadways to operate completely efficiently. By overreacting to avoid collisions, often by decelerating too abruptly, a driver can cause a wave of cars to slow down sequentially, forming a queue, and creating what is known as an "emergent traffic jam". This in practise occurs when drivers will, upon reaching the end of the jam, have observed no visible cause for the traffic! In a densely-packed road, emergent traffic jams are only fully alleviated when the volume of cars on the road decreases sufficiently; therefore, they can cause significant delays for all of the cars that follow on the road.

In many ways, the need for more safety in driving, as well as the desire to reduce overall commute time, along with the rise of automation and artificial intelligence, has led to the creation of Autonomous Vehicles (AVs). Currently, there are many cars on the road which are known as "almost self driving", including the Tesla Model 3 and Nissan Leaf. These cars have the advantages of having more sensors, for example with LIDAR, or a wider visual understanding of the road, for example, by using image recognition, and can also process a larger amount of historical data that can guide 
real-time decisions while driving.

A road fully-populated with AVs, along with being much safer to travel on, is also likely to be nearly free of traffic jams, because coordinating a series of vehicles with different exits becomes a network flow problem with near-perfect theoretical conditions. Mixed-autonomy roads, the roads shared by both AVS and human drivers, combined with completely effective collision-free technology, defines the future of transportation. But will AVs also reduce congestion and therefore improve the traffic flow of such collision-free mixed autonomy roads?

\subsection{Motivation of the Thesis}

The majority of the economic cost that exists as a result of traffic occurs on highways, especially in rush hour, when roads become densely packed. In any situation where a large group of objects are travelling in the same direction, using a shared passage, and the flow-rate of the objects is larger than the throughput of the passage, traffic is bound to occur. But traffic can be exacerbated by drivers behaving unnecessarily aggressively to maximize their short-term speed, because of the stop-and-go waves created, which, in turn, impact the rest of the drivers that are further behind on the road. The primary decisions that are frequently being made while driving on a highway are whether or not to change lanes, and whether or not to change speeds. Drivers make these decisions by constantly assessing how fast their cars can travel to minimize the total driving time, but also in relation to the amount of risk that they are willing to take. Drivers do not receive, and therefore rarely factor in macro-level knowledge of the entire road when making these decisions!

The first AVs will strongly prioritize safety, and therefore, will likely only store the relevant inputs needed to ensure that collisions do not occur. Therefore, in

mixed autonomy roads, the models used to mitigate emergent jams may need to be constructed explicitly using information necessary for ensuring safety, so as to account for the limited understanding of the intention of neighbouring human drivers. The advantages that $\mathrm{AVs}$ possess when making safe lane and speed decisions to improve traffic flow, are that they can retain and make use of their history of experiences. 
These experiences include, specifically, what was visible to the vehicle at the time the decisions were made, such as gaps to adjacent cars, any potholes or construction zones, etc., and also what actions were taken after making those observations, both in terms of lane and speed choice. They also have the ability to share these experiences with other AVs on the road, and therefore, can, to some extent, approximate macrolevel road dynamics. This creates a need for complex models, specifically Machine Learning (ML) models, that can develop a heuristic for how to behave, given the local observations of the road.

Traditionally, ML has been dominated by two sub-fields, referred to as supervised and unsupervised learning. Supervised learning involves learning from a set of labelled training examples, where the labels for each series of input features represents the "correct" behaviour or outcome, and the goal is to develop a model that can generalize to similar experiences that do not exist directly in the training set. This is not solely adequate for AVs, because even though there are ways to interpret how good a given decision taken was, there is no reliable method to determine what the exact optimal behaviour would have been. Unsupervised learning, on the other hand, is used to find the structure hidden in collections of unlabelled data. This is closer to what the AVs need to do, but still it alone is not sufficient, because a set of optimal decisions does exist, and it is necessary to predict the quality of each decision made as accurately as possible in order to optimize future decisions. The method of modelling used for road situations must incorporate the idea of dealing with unlabelled data, but still attempt to make optimal decisions.

Reinforcement Learning (RL), a third sub-area of ML, is used traditionally to train computers to perform well in game settings such as Chess (Deep Blue, made by IBM) or Go (AlphaGo, made by Google), and many other video and board-games. Indeed, RL provides an ideal class of solutions for our problem at hand. In RL, an agent, receives a set of local observations from the global environment, and then chooses from a finite set of actions, with the intention of maximizing a numerical reward signal cumulatively over the duration of the experiment. In the situation involving training $\mathrm{AVs}$ to minimize delays due to traffic, the agent is the car, the local observations are the gaps to neighbouring cars, the global environment is the 
full road, the actions are the lane and speed choices, and the reward signal can be selected as an operation of the gaps or speeds of the individual car or an aggregate of all $\mathrm{AVs}$ on the road.

The output of RL is that of determining which actions are to be selected given the state observed. But the underlying dilemma is the trade-off known as exploitation versus exploration. Exploitation refers to the task of selecting sequences of actions that, based on recent history, have been shown to produce the maximum cumulative reward. On the other hand, exploration involves sacrificing some known larger immediate rewards for the potential of receiving a higher long-term cumulative reward. This is, clearly, related to the situation encountered in driving. For example, taking the maximum speed possible on every iteration could lead to the largest immediate reward on that iteration, which seems like an intuitively obvious decision, but it may be more beneficial in the long term if cars prematurely slowed down to avoid suddenly braking in the future. In mixed-autonomy roads, especially those with lower percentages of AVs, RL can take advantage of the communication and memory of $\mathrm{AVs}$, even if each AV is given a limited visibility of the full road, and therefore will eventually lead to the improvement of travel conditions.

\subsection{Objectives of the Thesis}

The main objective of this thesis is to explore the traffic patterns of mixed-autonomy collision-free highways, with AVs designed to mitigate emergent jams, using RL. The first steps involve understanding the conditions for roads containing only human drivers. This is followed by replacing a small percentage of these drivers with AVs, and re-assessing the road conditions. Finally, we determine how road conditions continue to improve as the percentage of $\mathrm{AVs}$ increases.

The approach taken in our research was to develop a road traffic model, specifically a micro-traffic model, that controls the speed and lane decisions for each car, on each iteration. In this way, the AVs could be tested in a live traffic simulation. Human drivers are simulated using rules which are inherently stochastic, and also enforce collision-free driving conditions. Furthermore, in this micro-traffic modelling 
framework, all vehicles receive only the gaps between themselves and adjacent cars, to determine the speed which they can take on the next iteration. This thesis will be limited to single and double lane roads, including both situations where cars do and do not enter or exit the road. However, the modelling framework developed to simulate road traffic is built for $n$-lane roads, and some results for 3 and 4 lane roads, which only involve human drivers, are also included.

\subsection{Contributions of the Thesis}

The contributions of this thesis are as follows:

- We have surveyed the fields of micro-traffic road models, RL, and the applications of RL to train AVs for highway settings.

- We have identified that the majority of the published literature does not provide AVs with the same field of inputs as the human-driven vehicles. It also does not address the impact of AVs in terms of reducing traffic jams in mixed-autonomy roads.

- We have developed a traffic modelling framework, inspired by Nagel-Schreckenberg micro-traffic modelling, that manages the lane and speed decisions made by every car on every iteration. This includes allowing for roads with different numbers of lanes, roads that both permit and do not allow for the entry or exit of cars, and roads with random patches of slower maximum allowed speeds so as to simulate construction zones or temporarily blocked lanes.

- We have designed a modelling system to simulate human drivers, separating them into Passive, Normal, and Aggressive types to model diverse driving behaviours.

- We have experimentally determined that the simulation of human drivers underperforms the theoretically optimal conditions in single lane roads with no entries or exits that are densely packed, and also for many different double lane roads. 
- We have used a feature set, derived from the exact same inputs that human drivers use when making decisions, to train AVs with Deep Q Networks for single and double lane roads. In almost all situations, and with any percentage of AVs, results from this approach outperform the results from roads containing solely human drivers. Furthermore, these results also show that the human drivers on the road travel faster with the presence of AVs. For the most part, these trends are amplified as the percentage of AVs increases. Some highlights of the results are as follows:

- In single-lane roads, the average speed of all cars on the road was increased by $10 \%$ to $23 \%$ and the percentage of stopped cars on average per iteration was decreased by $14 \%$ to $65 \%$, after $5 \%$ to $50 \%$ of the vehicles were converted to AVs.

- In double-lane roads with no entries or exits, the average speed was increased by $5 \%$ to $26 \%$, and the percentage of stopped cars was decreased by $25 \%$ to $69 \%$, after $5 \%$ to $50 \%$ of the road was converted to AVs.

- In double-lane road containing entries and exits, the average speed was increased by $24 \%$ to $44 \%$, and the percentage of stopped cars was decreased by $20 \%$ to $68 \%$, after $5 \%$ to $50 \%$ of the road was converted to AVs.

- The work done has led to one research paper:

- Maheshwari, A. and Oommen, B.J., Reinforcement Learning for Reducing Congestion in Mixed Autonomy Highways. To be submitted.

\subsection{Organization of the Thesis}

Chapter 2 presents an overview of the literature. The problem being solved involves three relatively distinct fields: micro-traffic road models, RL, and the application of RL to model AVs in highway settings. The specific road modelling framework used in the thesis is based on Nagel-Schreckenberg (NS) traffic modelling, and therefore, this framework is initially analyzed, in detail. Next, the underlying algorithms eventually 
used to model AVs in this thesis, known as model-free RL, leading up to and including Deep Q Networks, is explained. This is the approach used in many well-known AIbased video game applications, but has recently begun to gain popularity in realworld problems that involve learning from interaction, specifically where supervised and unsupervised ML are not efficient or adequate. Finally, while there was a lot of prior research on modelling AVs using RL, and not much previous work that directly focused on using RL in mixed-autonomy roads to mitigate traffic jams, some of the most related existing research was examined. This included papers that involved either improving mixed-autonomy traffic using AVs that had a complete knowledge of the entire road at all times, using RL to model AVs to mitigate congestion that occurred due to lane blockages, and using RL to program a single AV to efficiently navigate obstacles on the road.

Chapter 3 defines the road modelling framework for all the traffic experiments conducted in this thesis. It also discusses the specific criteria that will be tracked to assess the conditions of the roads in any experiment. The section on road modelling defines: the specific data structure which represents the road system, how the road is updated on each iteration, how cars are represented, and how cars traverse, enter, and exit the road, in general. The section discussing criteria lists 10 different statistics which will be monitored on each iteration, but also separates them into three levels of importance: the first level focuses on the average road speed, the second level focuses on the persistence and duration of emergent traffic jams, and the final level focuses on all other factors including lane changing behaviour and the spacing of the cars.

Chapter 4 determines the baselines of the road criteria developed in Chapter 3 , for experiments containing exclusively "human drivers", which are referred to as Stochastic Vehicles (SVs). The chapter begins by stating the algorithms used by SVs to choose their lane and speed on each iteration, and specifically focuses on how these rules are designed to ensure that vehicles stay collision free, while still allowing the them to behave aggressively. The chapter concludes with experimental results for single and double lane highways. Results for the behaviour of SVs in 3 and 4 lane highways as well as some more interesting results are given in Appendix B.

Chapter 5 evaluates the impact of AVs in mixed autonomy roads, specifically 
compared to the baselines developed in Chapter 4. The chapter begins by defining the RL environment, specifically by stating the actions and reward functions used to design the AVs. The chapter then continues by evaluating AVs in single lane roads and double lane roads. For single lane roads, many different RL algorithms were used, and in each corresponding sub-section, the state space and specifics of training are given. For double-lane roads, only deep Q networks, which provided the best results for single-lane roads, were used. The chapter finishes with a brief section reviewing the main results. The extensive results, in tabular form, are provided in Appendix A.2.

Chapter 6 concludes the thesis. It summarises the key results, includes a brief summary of each chapter, and discusses some of the potential ideas for future research. 


\section{2 \\ Literature Review}

\section{$2.1 \quad$ Introduction}

The objective of this thesis is to assess the impact of autonomous vehicles (AVs) on improving traffic flow in mixed-autonomy roads. This can be rephrased as a Reinforcement Learning (RL) problem, where a collision-free highway is the global environment, and the AVs serve as agents which collect local information about the gaps between themselves and adjacent cars. The action set for AVs involves both choosing which lane to travel on and what speed to travel at, and the corresponding rewards are obtained based on their ability to traverse the road as fast as possible. Therefore, the background to solve this problem involves sufficiently understanding three separate areas: micro-traffic modelling to simulate road traffic, covered in Section 2.2, followed by model-free RL, covered in Section 2.3, and finally, methods for modelling AVs to navigate road traffic using RL, covered in Section 2.4. 


\subsection{Micro Traffic Modelling}

Traffic occurs when a set of objects are travelling in the same direction on a shared passage to reach their respective destinations. This can occur when hemoglobin carries nutrients to an area of the body, when a large crowd is trying to enter or leave an event, or, as is studied in this thesis, when cars are travelling on a highway.

Increasing traffic flow is always possible by increasing the width, and therefore the throughput, of the passage the objects are sharing. This is often an extremely costly endeavour, and is rarely ever feasible. Without modifying the passage, another way to maximize flow is to restrict the speed of the objects travelling through the network so as to attain to the maximum throughput. However, calculating this optimal flow rate is non-trivial, especially when objects are constantly entering and exiting the passage and their destinations and intentions are not explicitly known to the system. Furthermore, objects tend to follow an $n$-periodic distribution, and therefore move in clumps rather than in a uniform pattern, suggesting that even after knowing the overall density, different sub-regions of the network can have different optimal behaviours.

Emergent traffic jams occur when a car overreacts to avoid a collision, usually by abruptly decelerating. This causes a cascade effect forcing the cars behind it to decelerate, as well. This "log-jam" of "slowing-down" cars will not return to normalcy until the overall density of the system decreases sufficiently [4]. Micro-traffic models provide a method of simulating the behaviour of these jams, which track the trajectory and decisions made by cars at every time interval. This section begins with a common framework of micro-traffic modelling, known as Nagel-Schreckenberg (NS) traffic modelling. In this thesis, a variant of this type of modelling is used to model human drivers. The Intelligent-Driver-Model (IDM) will also be briefly discussed because some of the previous work that models AVs to navigate road traffic uses it to manage speed decisions in their experiments, even though it is not used directly in this thesis. 


\subsubsection{NS Traffic Modelling}

This section begins by analyzing the two basic variations of the single-lane NS model $[10,11]$, followed by methods to extend the modelling to multiple lanes [14, 22]. Finally, some previous work assessing the impact of communication between vehicles $[5,6]$, how this can help mitigate traffic jams [4], and how this relates to the impact that AVs can have, is analyzed.

\section{Single-Lane NS Traffic Modelling}

In single lane NS modelling, the road is represented with a one-dimensional array. Each element of the array can be either empty if no vehicle is on that cell, or contain the speed of the occupying vehicle in the range $\left[0, v_{\max }\right]$, where $v_{\max }$ represents the speed limit. In this case, the speed represents the number of elements forward that the vehicle will move during one iteration. A collision occurs when two vehicles end up on the same cell of the array, and in NS modelling, the rules used to model each vehicle enforce collision-free settings. On every iteration, each vehicle determines its speed using the following steps:

1. The vehicle determines its Front Gap, $n_{\text {gap }}$, which is the number of cells between itself and the next vehicle.

2. If a vehicle is travelling at $v_{\max }$, and $n_{\text {gap }} \geq v_{\max }$, the vehicle continues travelling at the maximum speed.

3. Otherwise, the vehicle determines its speed, given its current speed, $v$, as follows: Acceleration: If $n_{g a p} \geq v+1$, then with probability $p$, the vehicle accelerates to $v+1$. Otherwise, it remains at $v$.

Maintain: If $n_{g a p}=v$, the vehicle maintains its current speed $v$.

Deceleration: If $n_{\text {gap }} \leq v-1$, the vehicle slows down to $n_{\text {gap }}$ with probability $q$. Otherwise it changes its speed to 0 , representing a sudden brake.

The randomization creates non-deterministic acceleration, and also leaves room for vehicles to decelerate more than is necessary, which simulates the overreaction 
that results in an emergent traffic jam. However, both methods for acceleration and deceleration can be improved to be more realistic, because the probabilities that influence these actions do not depend at all on the magnitude of the gap. For example, in a case where the Front Gap for a car is infinitely large, and the car is not travelling at maximum speed, the probability of a car accelerating should be much higher compared with another scenario where the Front Gap is closer to the current speed.

There is another version of the single-lane NS-model that is less compromised by this problem. In this model, all vehicles choose a speed that is exactly equal to the minimum of their Front Gap, their current speed plus one, and the speed limit on each iteration. Then, each vehicle can independently reduce its speed by one unit, on every iteration, based on a deceleration probability $q$, to add randomness. This can also be made more realistic by allowing vehicles to accelerate by more than one unit, and allow for $q$ to be dynamically modified. Such adjustments, which were not made in the original papers, [10] and [11], will be made in this thesis, and are explained further in Chapter 4.

A vehicle is deemed to be jammed when it is travelling at less than the speed limit, and accordingly, the lifetime of an emergent jam is measured as the number of iterations until the number of jammed cars is zero. To understand the properties of non-interacting jams, in the paper [10], vehicles were perturbed once the previous jam had finished. As a result of numerous experiments, the literature has shown, experimentally, using a braking probability $q=\frac{1}{2}$, that the lifetime distribution for jams which last more than 100 iterations follows the power law: $P(t) \sim t^{-(\delta+1)}$ where $(\delta+1)=1.5 \pm 0.01$.

The most important benefit of either of these modelling frameworks is that cars only need to determine their Front Gaps to make speed decisions. This is very realistic, because drivers know very little about exactly what the speed, aggressiveness, and acceleration of other drivers sharing the road; they can only make collision-free decisions for the next time step based on the gap observed at the current time. 


\section{Multi-Lane NS Traffic Modelling}

There are many lane change models available, but generally, the theme in NS with multiple-lanes is that a car will change lanes if the car is currently not travelling at the maximum speed, if there is an available adjacent lane which has a larger Front Gap, and if a safe lane change is available. To determine whether or not a safe lane change exists, the following rules are added:

1. The distance to the vehicle ahead in the current lane, $d$, is less than a predetermined safe length, $l$.

2. The distance to the vehicle ahead in the target lane to which the vehicle wants to changed to, $d_{\text {target }}$, is larger than a predetermined target length, $l_{\text {target }}$.

3. The distance to the vehicle behind in the target lane, $d_{\text {target }}^{-}$, is less than another predetermined safe length, $l_{\text {target }}^{-}$.

4. The target cell is not scheduled to be occupied. This constraint is required when, for example, in a three lane scenario, cars from lane 1 and lane 3 can both switch to the same location in lane 2 at the same time.

Choosing which lane to change to, assuming the constraints are met, depends on which lane has a higher Front Gap. The values for $l, l_{\text {target }}$, and $l_{\text {target }}^{-}$can be enforced to be collision free, i.e. by setting them as $l=\min \left(v+1, v_{\text {max }}\right), l_{\text {target }}=d$, and $l_{\text {target }}^{-}=v_{\max }[22]$. Such a modelling paradigm is still practical, because it only depends on the ability of drivers to view the Front and Back gaps of their adjacent lanes. The fourth constraint can be modelled more concretely, because it is possible to see two lanes adjacent across when no car is in the directly adjacent lane. Note that certain factors have not been considered, such as the stochastic aspect of lane changes based on driver preference, which is especially true in cases where cars can enter or exit the road.

There are methods to convert the NS model to a continuous scale, so that cars do not have to be arranged in a grid and can be of different lengths. Continuous modelling can have a larger impact in applications where a difference of feet (or even 
inches) can still permit a car to make safe and advantageous lane changes, including those that involve route planning, traffic lights, and vehicles of different sizes. The majority of those methods allow one vehicle to cover multiple adjacent road cells at the same time, and where vehicles have a predetermined required minimum amount of cells between two cars to maintain safety. These scenarios will not be analyzed in this thesis because, in a highway setting, a difference of a few feet or inches does not intuitively impact the speed and lane changes on average, the way it would in a residential road. However, this idea is worth considering for future work.

Next, a variation of NS modelling where vehicles are able to perform limited communication with adjacent vehicles, known as the Extended NS algorithm (ExNS), and how this can change road dynamics, is discussed. This will also give some insight into how AVs, which have a larger view and longer memory of traffic states, can potentially help mitigate traffic jams.

\section{Extended NS Traffic Models}

In the NS modelling discussed in the previous section, vehicle $i$ is aware of its speed, $v_{i}(v(t))$, and Front Gap with the vehicle ahead, $v_{i}(d(t))$. In ExNS, cars are also given the ability to observe the Front Gap and the current speed of the vehicle immediately ahead, $v_{i+1}(v(t))$, and $v_{i+1}(d(t))$ [5]. Then, if $v_{i}(d(t)) \leq v_{\max }$, the vehicle chooses its speed using a Front Gap equal to the minimum of $v_{i}(d(t)), v_{i+1}(v(t))$, and $v_{i+1}(d(t))$. By working in this manner if the vehicle ahead is not close to the current vehicle, the current vehicle will accelerate to the speed limit, until it catches up to the vehicle ahead. After that, it will make a speed update using the information of the vehicle ahead, so long as it does not collide with the vehicle ahead.

Experimentally, it has been shown that overall traffic is much smoother when vehicles follow ExNS. There is a further extension of ExNS called ComNS, where drivers can view the speeds of all cars inside a pre-specified distance, which has shown the ability to reduce the impact of emergent jams [6].

Experimental work has also been done to show how "traffic reducer agents" can mitigate congestion on multi-lane highways, specifically when two consecutive reducing agents are placed on a single or double lane road [4]. In the experiments run in the 
associated paper, reducer agents follow ExNS rules when there is no congestion. Once congestion is identified, if the velocity of the car ahead is within a pre-specified range, the car ahead is within a pre-specified distance, and the velocity of the current car is non-zero, the car decelerates by a single unit of speed. This allows for the vehicle to prematurely slow down by a fixed amount, lowering the likelihood it will need to suddenly brake in the future. When all other cars follow the basic NS modelling and double lane modelling given above, it has been shown that just two reducer agents placed consecutively noticeably improve average road speeds [4].

These models are not realistic, because unless cars are able to communicate, there is no reasonable way to consistently determine the gaps observed by the vehicle ahead. However, they do offer some insight into how AVs can help mitigate emergent jams. Because AVs have the ability to "remember" past experiences of traffic, and can share experiences and state spaces with each other, it is possible for them to approximate the current speed and forward gap of the vehicle ahead, by measuring the changes in Front Gap between iterations, especially in the single lane case.

\subsubsection{IDM Traffic Modelling}

The Intelligent Driver (IDM) car-following model contribute another method of simulating human drivers, but involve a continuous road space rather than one with discrete cells. Cars use their relative distance and velocity with adjacent cars to determine how much to accelerate on a given iteration. The acceleration for vehicle $\alpha$ is determined using its distance to the vehicle ahead $s_{\alpha}$, velocity $v_{\alpha}$, relative velocity $\Delta v_{\alpha}$, and the pre-specified desired headway $s^{*}$, using:

$$
a_{I D M}=\frac{d v_{\alpha}}{d t}=a\left[1-\left(\frac{v_{\alpha}}{v_{0}}\right)^{\delta}-\left(\frac{s^{*}\left(v_{\alpha}, \Delta v_{\alpha}\right)}{s_{\alpha}}\right)^{2}\right]
$$

This model also accurately simulates the actual behaviour exhibited by drivers [16]. The largest advantage of this model is the fact that vehicles can be of different lengths, and can, therefore, use a continuous representation of the road. 


\subsection{Reinforcement Learning}

The human brain is exceptional at learning from interaction with an environment. This is, in part, due to its abilities to assess the importance of information, and summarize experiences quickly. Computers, on the other hand, are optimized for storing and accessing specific information from large pools of memory and repetitively performing computations accurately. Reinforcement Learning (RL) is the study of computational approaches to learning from interaction, with the specific goal of maximizing cumulative numerical reward signals provided from an environment, by making an optimal sequence of decisions under uncertainty. While this field attempts to solve the same "goals" as a human learning from interaction, the methods used take advantage of the strengths of a computer. Therefore, while motivated by the brain, RL is not provably closely related to how the brain performs learning.

This section begins by presenting the formal mathematical RL problem and how this can be represented by a Markov Decision Process. This is followed by introducing a class of algorithms known as Temporal Difference Learning, which are currently the most widely-used algorithms in RL applications [15]. The section finishes with an overview of traditional supervised machine learning (ML) algorithms, and methods for leveraging supervised ML, specifically Deep Neural Networks (DNN), to improve RL.

\subsubsection{The Reinforcement Learning Problem}

In $\mathrm{RL}$, an agent, the learner, executes actions from a finite set of actions, $A_{t} \in A$, on discrete time steps, $t$. After an action is executed, the environment, with which the agent is interacting, responds with a numerical reward, $R_{t}$, which informs the agent how well it performed on the previous time step. The environment also provides a collection of observations local to the agent, known as a state, $S_{t}$, derived based on the action the agent previously took, and which constrains and influences the actions that the agent can take on the next time step.

The sequence of interactions is repeated indefinitely unless the agent reaches a 
terminal state or until it reaches a pre-determined time limit. The resulting sequence of actions, states, and rewards, $S_{0}, A_{1}, R_{1}, S_{1}, A_{2}, R_{2}, S_{2}, \ldots$, is referred to as an "episode". The goal of the agent is to maximize cumulative received rewards by selecting an optimal series of actions, and to accomplish this, the agent often needs to experience many episodes. An agent is defined by at least one of the following:

1. Policy: The mapping specifies the actions to be taken from each state:

$$
\pi(a \mid s)=P\left[A_{t}=a \mid S_{t}=s\right] .
$$

2. Value Function Estimate: The expected future discounted reward from each state, while following policy $\pi$ :

$$
v_{\pi}(s)=E_{\pi}\left[R_{t+1}+\gamma R_{t+2}+\gamma^{2} R_{t+3}+\ldots \mid S_{t}=s\right]=E_{\pi}\left[\sum_{k=0}^{\infty} \gamma^{k} R_{t+k+1} \mid S_{t}=s\right] .
$$

3. Model: An agent's representation of the environment, both for predicting which state and what reward will come next after taking an action from a state. This is represented by the state transition probability and the reward function:

$$
P_{s s^{\prime}}^{a}=P\left[S_{t+1}=s^{\prime} \mid S_{t}=s, A_{t}=a\right] ; R_{s}^{a}=E\left[R_{t+1} \mid S_{t}=s, A_{t}=a\right] .
$$

The two major sub-problems in RL are known as prediction, which involves estimating the value function in order to help an agent determine which states are expected to maximize reward, and control, which involves determining the best actions to choose from each state to obtain this maximum expected reward (in other words learning the best policy). In solving both prediction and control, there is a fundamental trade-off between exploring new and currently less optimal actions, in case they have a delayed reward, and exploiting the currently-known model of the environment, to capitalize on actions that have proven to give known higher rewards in the limited experience acquired. This trade-off between exploration and exploitation is what makes RL a unique and very challenging learning problem. The prediction and control problems can be formally represented by a Markov Decision Process. 


\subsubsection{Markov Decision Processes}

Both the prediction and control problems are heavily dependent on the definition of a "state", provided by the environment. A state is known as a Markovian (or Markov) state if the probability of entering a new state on a given time step only depends on the current state: $P\left[S_{t+1} \mid S_{t}\right]=P\left[S_{t+1} \mid S_{1}, \ldots, S_{t}\right]$. This suggests that an agent does not need to "remember" which states it has travelled to in the past to predict which actions or rewards are to be taken or received in the future. If the agent is able to fully observe the environment, and all states are Markov States, the problem is known as a Markov Decision Process (MDP). Note that by defining the current state as the history of all state-action-rewards thus far, therefore representing the full history, as long as there are a finite total number of possible state-action-reward combinations, all states will satisfy the Markov property. Therefore, most problems can be represented as MDPs.

The MDP is effectively a set of state transition matrices, each of which stores the transition probabilities from all states $s$ to all successor states $s^{\prime}$, for each action from the finite set of actions. The return, $G_{t}$, for an MDP at any time step, $t$, is the discounted sum of future rewards:

$$
G_{t}=\sum_{k=0}^{\infty} \gamma^{k} R_{t+k+1}
$$

Therefore, the associated state-value function, individually for each action in the MDP, can be decomposed into a sum of the immediate reward received, and the discounted value of the future successor state, known as the Bellman equation:

$$
v(s)=E_{\pi}\left[G_{t} \mid S_{t}=s\right]=E\left[R_{t+1}+\gamma v\left(S_{t+1}\right) \mid S_{t}=s\right]=R_{s}+\gamma \sum_{s^{\prime} \in S} P_{s s^{\prime}} v\left(s^{\prime}\right)
$$

Incorporating the agents ability to select actions, the associated action-value function and its associated Bellman equation are:

$$
q_{\pi}(s, a)=E_{\pi}\left[G_{t} \mid S_{t}=s, A_{t}=a\right]=E_{\pi}\left[R_{t+1}+\gamma q_{\pi}\left(S_{t+1}, A_{t+1} \mid S_{t}=s, A_{t}=a\right)\right]
$$

The prediction and control problems are formally solved by determining the policy which maximizes the Bellman equations for the state-value and state-action value 
functions: $v_{*}(s)=\max _{\pi} v_{\pi}(s)$, and $q_{*}(s, a)=\max _{\pi} q_{\pi}(s, a)$, respectively. This is a system of linear equations, and therefore a unique solution can be determined in $O\left(n^{3}\right)$ time, where $n$ is the number of unique states or state-action pairs. However, this requires that all possible state transition probabilities are available, along with their desirability in terms of their expected rewards. Assuming those requirements are met, the computation power to compute the direct solution must exist, which is unlikely for problems that have an infinitely-finite amount of state-action pairs.

In summary, the problem posed by an MDP can be summarized using the following set: $\left[S, A, P, R, \pi_{0}, \gamma, H\right]$, where $S$ is the state space, $A$ is the action space, $P$ is the set of transition probabilities, $R$ is the reward function, $p_{0}$ is the initial policy or state distribution, $\gamma$ is the discount factor, and $H$ is the time horizon, with the goal of optimizing a stochastic policy to maximize the discounted return.

In case a direct solution is not feasible, for the reasons listed above, an agent must be able to learn an underlying model and/or determine a sequence of actions iteratively from experience. This is done using model-free methods, the most common of which is known as Temporal Difference Learning.

\subsubsection{Temporal Difference Learning}

A Markov process heavily depends on a model of transition probabilities, and such a model must either be provided explicitly, or calculated iteratively as experiences accumulate. However, in many cases, by the time enough experience is acquired that a reliable set of transitions exists, the episode will have already ended!

To account for this, the agent must achieve optimal action selection iteratively while experience is being acquired, independent of a model of the transition probabilities. The most widely applicable version of model-free methods used is known as Temporal Difference Learning (TD Learning), in which the prediction and control problems are solved separately. 


\section{Model-Free Prediction}

In the prediction problem, the objective is to estimate the state-value function for all non-terminal states while following policy $\pi$, and discount factor $\gamma$, represented by: $v_{\pi}(s)=E_{\pi}\left[G_{t} \mid S_{t}=s\right]$. Without a model of the reward process, the only way to do this is to continually improve on the state-value function estimate as more rewards are experienced.

When the training process can be separated into many episodes, one method of value function estimation is to wait until an episode terminates, and then to update the overall state-value function based on the returns experienced after each visit to each state observed during the episode. Given a count of the number of visits to each state, $N\left(S_{t}\right)$, and a sum of the returns experienced from each state during the episode, $G_{t}$, the estimate of $v_{\pi}, V$, can be updated as follows:

$$
V\left(S_{t}\right) \leftarrow V\left(S_{t}\right)+\frac{1}{N\left(S_{t}\right)}\left(G_{t}-V\left(S_{t}\right)\right)
$$

By the law of large numbers, as the number of episodes approaches infinity, if the underlying process is stationary, this will converge to $v(\pi)$.

In a non-stationary or non-Markovian process, where the unknown reward distribution can change as time progresses and therefore the frequency of visits to each state is not as valuable, instead of using the count, one can substitute a learning rate $\alpha$, to get a new update equation, known as a Monte-Carlo update:

$$
V\left(S_{t}\right) \leftarrow V\left(S_{t}\right)+\alpha\left(G_{t}-V\left(S_{t}\right)\right)
$$

The state-value update happens in a direction that corrects the error between the actual return observed and the value of the expected return predicted. Observe that if $\alpha$ is 1 , it implies replacing the old value entirely with the new return. This method of updating uses the actual return experienced on each episode to correct the state value estimate, and it is therefore completely unbiased. Specifically if the underlying process is non-stationary, or non-Markovian, this method will be effective, because the Monte-Carlo prediction converges to the minimum MSE estimate of the statevalue function based on the rewards experienced. However, it is an offline update, because the agent cannot improve its estimate until the end of the episode. In cases 
where a very limited number of episodes are available, or where a particular state is visited very infrequently, this solution will have a high variance. Therefore, another method to learn incrementally, but fully online, is required.

$\mathrm{TD}(0)$ methods address this need, and work by waiting just one time step in any episode, and then updating the current value based on the new reward received and the discounted existing estimate of the value function at the new state observed:

$$
V\left(S_{t}\right) \leftarrow V\left(S_{t}\right)+\alpha\left(R_{t+1}+\gamma V\left(S_{t+1}\right)-V\left(S_{t}\right)\right)
$$

Future updates only require a single time-step and therefore do not need to wait for the episode to end! However, since it is updating the current estimate of the value function based on a previous estimate, known as bootstrapping, this can very quickly lead to biased predictions. $\mathrm{TD}(0)$ is effectively building a maximum-likelihood Markov model step-by-step, as experience is acquired.

For both of these methods, the common idea is to update the existing value function in the direction of the TD error, $\delta_{t}$, which represents the difference between the TD target, and the previous value-function estimate. The TD error for TD $(0)$, for example, is defined as $\delta_{t} \leftarrow R_{t+1}+\gamma V\left(S_{t+1}\right)-V\left(S_{t}\right)$. Both sets of predictions also self-correct themselves as more experience is acquired. However, TD(0) does not perform well compared to Monte Carlo methods in situations where the underlying environment is non-Markovian, because it gets quickly biased from initial experiences in new states, and as a result, it takes much more time to self-correct itself.

Clearly, there is a middle-ground between looking 1-step ahead in $\operatorname{TD}(0)$, and waiting until the end of the episode in Monte-Carlo. For example, a 2-step return updates using a TD-error: $\delta_{t} \leftarrow R_{t+1}+\gamma R_{t+2}+\gamma^{2} V\left(S_{t+2}\right)-V\left(S_{t}\right)$, and similarly, a 4-step return will receive four time steps worth of rewards and then use the value function prediction for the fifth time step onward. In fact, the best prediction would be to average the expected returns for all $n$ steps ahead, as this will ideally solve both the bias and the variance problems existing in $\operatorname{TD}(0)$ and Monte-Carlo updates.

To accomplish this, a decay factor, $\lambda \in[0,1]$, is introduced to determine how much to proportionally weigh short-term versus long-term returns. This creates a 
geometric series of returns, known as the $\operatorname{TD}(\lambda)$-return:

$$
G_{t}^{\lambda}=(1-\lambda) \sum_{n=1}^{\infty} \lambda^{n-1} G_{t: t+n}=(1-\lambda) \sum_{n=1}^{T-t-1} \lambda^{n-1} G_{t: t+n}+\lambda^{T-t-1} G_{t}
$$

In these returns, the first part of the sum applies until the episode terminates, and the second part is the weight given to the final return after termination. This decomposition of the sum shows that if $\lambda$ approaches 0 , this is exactly the $\operatorname{TD}(0)$ algorithm, and if $\lambda$ approaches 1 , by the end of the episode this will be close to equivalent to the Monte-Carlo update [15]. Combining this with the existing TD methods, creates a method known as forward-view $\operatorname{TD}(\lambda)$, which repeatedly performs the update:

$$
V\left(S_{t}\right) \leftarrow V\left(S_{t}\right)+\alpha\left(G_{t}^{\lambda}-V\left(S_{t}\right)\right)
$$

However, this still requires the agent to wait until the end of the episode to update the current weights based on the average observed return at each time step, and also requires the agent to store all future predicted returns on every time step, which is not feasible! Therefore, instead of waiting until an episode finishes, a memory vector is updated on each time step for each state individually, known as an eligibility trace, $E_{t}(s)$. The eligibility trace summarizes the state-value function up to the current time step, and then on each time step, it is decayed both by $\gamma$, the discount factor, and $\lambda$, the decay factor, such that: $E_{0}(s)=0$, and

$$
E_{t}(s)=\gamma \lambda E_{t-1}(s)+1\left(S_{t}=s\right)
$$

The updated value function for each state, $s$, uses the $\operatorname{TD}(0)$-error, $\delta_{t}$, and the eligibility trace $E_{t}(s)$ :

$$
V(s) \leftarrow V(s)+\alpha \delta E_{t}(s)
$$

The advantage of this approach is that credit is assigned to states that are both visited the most frequently, and visited the most recently, taking advantage of the benefits of both $\operatorname{TD}(0)$ and Monte Carlo methods. These $\operatorname{TD}(\lambda)$ methods are sufficient for solving the prediction problem [15], and fortunately, they translate directly to solving the control problem as well. 


\section{Model-Free Control}

Model-free control can be divided into two categories: on-policy and off-policy. In on-

policy, the idea is to evaluate the current policy being followed by directly estimating the action-value function. In off-policy learning, the goal is to estimate the value of a policy $\pi$, while following experiences sampled from a different policy, $\mu$. Off-policy methods are important when the optimal policy is unknown, or even when the policy is known but too complicated or computationally expensive, making it unusable.

Determining the optimal set of actions to take from each state is accomplished by updating the action-value function, $Q(s, a)$. At each time step, depending on which state is reached, the action which provides the highest long-term expected reward from each state is chosen. Furthermore, to avoid repeatedly picking the best known action given the existing experience, and therefore not performing sufficient exploration, an additional exploration parameter $\epsilon$ is used, such that with the probability $\epsilon$, a random action is chosen. The GLIE theorem states that $\epsilon$-greedy action selection will only improve the existing policy [15].

Given an existing policy, to update the action-value function, the value of the known next step following the current policy is used to update the present value:

$$
Q(S, A) \leftarrow Q(S, A)+\alpha\left(R+\gamma Q\left(S_{t+1}, A_{t+1}\right)-Q(S, A)\right)
$$

This is known as SARSA, because the original State-Action value is updated using the received Reward, and prediction of the new State, and next Action taken. These updates will converge, as long as the learning rate satisfies the two properties: $\sum_{t=1}^{\infty} \alpha_{t}=\infty$ and $\sum_{t=1}^{\infty} \alpha_{t}^{2}<\infty$.

To remove the need for a policy, Q Learning is used. In Q Learning, new actions are chosen using an $\epsilon$-greedy action selection method, which involves choosing the action with the maximum possible Q-value from the current state, to update the current state-action value:

$$
Q(S, A) \leftarrow Q(S, A)+\alpha\left(R+\gamma \max _{a^{\prime}} Q\left(S^{\prime}, a^{\prime}\right)-Q(S, A)\right)
$$


This can also be augmented to include multiple future returns by creating a $\lambda$-return:

$$
q_{t}^{\lambda}=(1-\lambda) \sum_{n=1}^{\infty} \lambda^{(n-1)} q_{t}^{(n)}
$$

The accompanying eligibility trace, to make this fully online, is: $E_{0}(s, a)=0$, and $E_{t}(s, a)=\gamma \lambda E_{t-1}(s, a)+1\left(S_{t}=s, A_{t}=a\right)$. The action-value function only increases if the state-action pair is experienced, and the eligibility trace now needs to store values for every state-action pair, instead of just for every state.

In summary, Q Learning provides a fully online and model-free way to maximize rewards without requiring an existing policy. However, as the number of states and actions grows larger, sufficient experience for all state-action pairs will take longer or potentially never be acquired. In fact, one of the most well-known RL applications, AlphaGo, has $10^{172}$ states, which is unlikely to ever be feasibly solved by maintaining a large table. Similarly, in the traffic setting, a state can be composed of multiple consecutive traffic "snapshots", or a collection of relative gaps to other vehicles over the past few iterations, which has a finite but not necessarily known amount of states. Therefore, it becomes necessary to abstract states into a set of features with weights that influence action selection, and then to update these weights based on the rewards received and feature values of the new state entered.

\subsubsection{Deep Q Learning}

This subsection starts with the abstraction from state and state-action value table updates to state and state-action value function approximation. This is followed by how supervised machine learning, including DNNs, can be combined with Q Learning to create a more effective RL approach.

\section{Q Learning with State Space Abstraction}

Until this juncture, all state-value functions used to solve the prediction problem, and state-action-value functions used to solve the control problem, involved updating a Q table which holds the quality, or desirability, of each state or state-action pair. In scenarios where the state space grows very large, this table-update will not be efficient 
or easily usable. As a result, instead of retaining values for each state or state-action, states and actions will be represented by a set of features, each with a corresponding weight, such that the features can differentiate the different states.

More specifically, the goals are now to learn the weights, $w$, to find the optimal $\hat{v}(s, \boldsymbol{w})$ to approximate $v_{\pi}(s)$, and $\hat{q}(s, a, \boldsymbol{w})$ to approximate $q_{\pi}(s, a)$. Instead of directly updating a specific state or state-action value, every time a new experience is seen, the corresponding state or state-action is represented by a set of features, and these features are processed by a function approximator to determine the corresponding Q-values. Also, on every time step, the weights used by the approximator for each feature are updated based on the reward and state transition which is received at the new time-step.

Assuming that the different features, $x$, are linearly combined, such that $x(S)=$ $w^{T} x$, then the new $\operatorname{TD}(0)$ error, where $\hat{v}\left(S^{\prime}, w\right)$ is the discounted estimate of the value function going forward, and $\hat{v}(S, w)$ is the previously outputted value function approximation, is:

$$
\Delta w=\alpha\left(R+\gamma \hat{v}\left(S^{\prime}, w\right)-\hat{v}(S, w)\right) \nabla_{w} \hat{v}(S, w)=\alpha \delta x(S)
$$

Similarly, for $\operatorname{TD}(\lambda)$, the gradient used for updates is $\Delta w=\alpha \delta_{t} E_{t}$, where the eligibility trace, $E_{t}$, is defined as: $E_{t}=\gamma \lambda E_{t-1}+x\left(S_{t}\right)$, and the error term, $\delta_{t}$, is defined as: $\delta_{t}=R_{t+1}+\gamma \hat{v}\left(S_{t+1}, w\right)-\hat{v}\left(S_{t}, w\right)$. In the control problem, the $\mathrm{Q}$ values are replaced in a similar fashion, and the action taken by the agent is chosen $\epsilon$-greedily. This method is known as linear value function approximation, and is effective given the right set of features.

An additional benefit to abstracting states with features is that the experience learned from one state will now directly and proportionally apply to all other states, depending on how similar their feature values are. However, this can be improved further by re-using past experiences and incorporating supervised learning instead of doing a single update using only the latest experience. 


\section{Incorporating Supervised Learning Methods}

Currently, the corresponding gradient is calculated and the weights are updated as each experience is gained, and all future updates do not re-use that experience. Experience replay is the idea of storing each state-action-reward-newState transition, $s_{t}, a_{t}, r_{t+1}, s_{t+1}$, in a replay memory, $D$, which stores the full history of the transitions, and then sampling a mini-batch of transitions from $D$, to update the weights on every iteration. The weights are updated using a supervised learning algorithm, that uses the state features and previous action taken as inputs, and the reward received plus predicted value from the new state multiplied by a discount factor as the output, or "label" [8].

Randomly sampling transitions removes the correlations between successive experiences, which is important to reduce the bias of the updates. Re-using past experiences also forces the agent to ensure that the weights, generated due to bootstrapping from the latest rewards, have not been changed to a point where the agent would now not be able to predict experiences similar to the past correctly [8]. This can be extended to concepts of prioritized experience replay, where past experiences that resulted in higher prediction errors, or that were determined to be more "important" experiences, are sampled more often. Usually, the size of the mini-batch remains fixed, which places a bound on the training time for each iteration.

There is a potentially significant problem with directly using experience replay, especially with a more complicated non-linear function approximator. Because the feature weights can change on every iteration, it means that both the prediction of future discounted reward in the target, $\hat{v}\left(S^{\prime}, w\right)$, and the current measure of error in the output, $\hat{v}\left(S_{t}, w\right)$, are changing at the same time. This can create large instabilities in the training process, because the gradient is being calculated at every time step using a changing target!

This is resolved by separating the function used to predict the discounted target value, known as a target function, from the output function, which is updated on every iteration. The target function contains fixed weights, $w^{\prime}$, that are updated on every $C$ iterations to the weights of the output function, $w$. This, effectively, means the output value is being updated based on old weights that do not incorporating 
the latest experience. However, as long as the weights in the target are updated frequently enough, it is a close enough approximation of the true future predicted reward. This has been shown empirically to stabilize the training process in many applications, and is known as using Fixed Q Targets [8].

In summary, the set of steps to accomplish Q Learning using function approximation with supervised learning are the following:

1. Determine feature values for the current state and send these values into a supervised learning algorithm, which returns all of the predicted action values.

2. Select which action to take using an $\epsilon$-greedy action-selection policy.

3. Perform the action in state $s$, receive a reward, observe the state $s^{\prime}$, and then store this transition, $\left[s, a, r, s^{\prime}\right]$, in the replay buffer.

4. Sample stored batches of transitions from the replay buffer and calculate the loss. For example, using a mean squared error loss function, the loss can be calculated as the squared difference between target and output function errors: $\left(R+\gamma \max _{a^{\prime}} Q\left(s^{\prime}, a^{\prime} ; \boldsymbol{w}^{\prime}\right)-Q(s, a ; \boldsymbol{w})\right)^{2}$.

5. Update the weights of the output function using the gradient between anticipated loss from the function approximator with fixed weights $\boldsymbol{w}^{\prime}$, and predicted loss from the output function.

6. After $\mathrm{C}$ iterations, replace the target weights with the output function weights.

In most Q Learning applications, the function approximator used in Step 1 is some form of a DNN, primarily because DNNs eliminate the need to manually build features, and also performs non-linear function approximations. DNNs have been heavily researched and used in a wide variety of applications; consequently, we only provide a very brief summary of the main ideas.

\section{Combining DNNs with Q Learning}

Linear function approximation assumes that the value function is a weighted combination of features, where each feature is a function of the states. Neural Networks (NNs) 
instead use many non-linear functions, known as activation functions, to map inputs to expected outputs. DNNs chain together layers of non-linear functions, therefore creating a larger number of distributed representations of the inputs, while still only requiring the same number of initial parameters [1].

The basics of any NN involve a dataset containing input features, $\mathbf{X}$, and an outputs which represent "correct" outcomes, $\mathbf{y}$, and repeating the following steps indefinitely until convergence [1]:

1. Draw a batch of training samples from $x$ and associated targets $y$.

2. Run each training sample through the network and obtain the predictions $y_{\text {pred. }}$.

3. Compute the loss, which is a measure of distance between the real targets $y$ and the predicted values $y_{\text {pred }}$.

4. Adjust the weights connecting the activation functions in the network to reduce the loss.

The first 3 steps are a set of simple computations, which of course, depend on the activation functions of each hidden neuron in each layer. The final step involves first taking the gradient with respect to the parameters of the chain of activation functions, which is done using backpropagation, and then adjusting the weights in the opposite direction of the gradient to reduce the loss on that particular input batch [1]. The methods for calculating the gradient are determined by the selected "optimizer", and the weights between each hidden neuron are adjusted by the learning rate, based on the calculated gradient. The most common variants of network architecture for Deep Q Learning are feed-forward NNs, and convolutional NNs [8], all of which are referred to as Deep Q Networks (DQNs).

Feedforward NNs can be represented as acylic graphs, beginning with inputs, and mapping to outputs, through a series of hidden layers. Each layer is known as a dense layer, where every neuron in one layer is linked to every neuron in the next layer by a weighted edge. In between, there are also dropout layers, which convert a random percentage of the weights between hidden layers to zero, forcing the network 
to generalize and not overfit on the training data [1]. In the cases of DQNs, typically there is one output representing the value of each possible action in the discrete space of actions.

In feed-forward NNs, every single feature from each sample is sent to each neuron of the first hidden layer, and all the representations captured by the first hidden layer are then transmitted to all future hidden layers. Therefore, the actual order of the input data is not accounted for, which can be advantageous in many scenarios where correlation between successive inputs can cause bias. However, in data formats where the actual order of input features is likely to be relevant in prediction, such as image data, text data, or time-series data, a convolution layer can be used in place of a dense layer.

Instead of inputting all features to every neuron in the hidden layer, a convolution layer receives a fixed-size window of consecutive input features from the previous layer, arranged such that the inputs overlap slightly from neuron to neuron, but not completely. Each convolution computes a set of learn-able filters, which can be interpreted as features of each patch, known as a feature-map. Convolution layers are also joined with pooling layers, which, similar to dropout layers, aggressively downsample feature maps, only retaining the most relevant and unique features from each representation and therefore forcing the network to avoid over-fitting [1]. These layers of convolution and pooling are finally combined with a fully connected layer, which similar to the feedforward network, is an action-value estimate for each action that the agent can take.

When applying either of these architectures to DQNs, the value used as the output, $y$, is the target value, which is the discounted estimate of future reward plus the actual reward received on the next time step, and is stored on each transition in the replay buffer. In summary, DNNs provide an ideal way to execute function approximation without requiring a large amount of knowledge about the state spaces, and also do not rely as heavily on the quality of individual input features. Next, some extensions to address issues with the Approximate Q Learning framework described above, will be explored. These extensions are not used directly in this thesis, but are included nevertheless, for completeness. 


\section{Extensions to Approximate Q Learning}

While Fixed Q Targets is sufficient in many situations to address the issue of maximization bias, Double Q Learning, which involves actively training two separate DQNs, is a more computationally intensive and robust solution [17]. In Double Q Learning, one network selects the maximum action: $a^{*}=\operatorname{argmax}_{a} Q_{1}\left(s_{1}, a\right)$, and the second estimates the value of the action $a^{*}: Q_{2}\left(s, a^{*}\right)$. This update can be represented as: $Q_{1}\left(s_{t}, a_{t}\right) \leftarrow Q_{1}\left(s_{t}, a_{t}\right)+\alpha\left(r_{t}+Q_{1}\left(s_{t+1}, \operatorname{argmax}_{a^{\prime}} Q_{2}\left(s_{t+1}, a^{\prime}\right)\right)-Q\left(s_{t}, a_{t}\right)\right)$. Furthermore, on any iteration, the role of $Q_{1}$ and $Q_{2}$ can be reversed.

Another potential issue with applying Approximate Q Learning, is that it is not always necessary to know the value of each action at each time-step. This occurs in states where, regardless of the action the agent takes, the environment will not be impacted. To separate the action value and state value, an advantage function is introduced: $A^{\pi}(s, a)=Q^{\pi}(s, a)-V^{\pi}(s)$. This is a subtraction of the approximate value function, $V$, used to solve the prediction problem, from the $Q$ value, used to solve the control problem [19].

In this scenario, the approximator will predict both the value function, and the advantage function subtracted by the mean advantage value across all actions, resulting in the following Q-update:

$$
Q(s, a ; \theta, \alpha, \beta)=V(s ; \theta, \beta)+\left(A(s, a ; \theta, \alpha)+\frac{1}{|A|} \sum_{a^{\prime}} A(s, a ; \theta, \alpha)\right)
$$

- This architecture is known as a Dueling Q Learning [19], and has been shown empirically to operate successfully in many game environments, where the only reward received is on the termination of the episode.

\subsection{Modelling Autonomous Vehicles}

There has been a lot of prior work done on designing AVs to navigate road traffic using RL. Models used to train AVs in previous works are differentiated based on the state space that the AV receives, the method the human-drivers are modelled by, how much the vehicles can observe from the environment, the reward function, the actions each vehicle is able to take, and what type of underlying $\mathrm{NN}$ is used. 
This section begins with covering two previous papers that analyzes the dynamics in mixed-autonomy roads, followed by prior work related to the mitigation of congestion using AVs, and finally, three prior examples covering the design of single AVs to navigate road traffic. There are many more examples in the existing literature that involve planning for AVs in many different capacities, but what is reviewed below has the most direct applications to what will be explored in this thesis.

\subsubsection{Mixed-Autonomy Road Traffic}

This subsection begins with one of the first examples of the mixed-autonomy traffic problem in which the AVs were only able to choose lanes, followed by a more recent paper that also investigates the mixed-autonomy traffic problem where cars choose both lanes and speeds. Both of these papers investigate the question of how different percentages of the cars on the road being autonomous impacts the overall average speed of the road.

\section{Lane Selection}

One of the first examples of navigating road traffic by combining NNs and RL, written in 1998, involved analyzing the ability for drivers to increase traffic throughput and more consistently maintain desired speeds by selecting lanes intelligently [9].

Cars were characterized as "selfish", if their strategy was to change lanes when a safe change was available and the car immediately in front was travelling slower than they were, simulating human drivers. On the other hand, cars were considered "smart" if they employed a cooperative lane selection strategy, simulating AVs. The state space for each smart car was its own current speed and desired speed, the relative speed of the surrounding 8 cars, whether each of the surrounding cars was smart or selfish, collectively constituting a state of 18 elements.

The performance function used for a set of cars was the squared difference between the actual and desired speed of all vehicles subtracted by the total number of lane changes per car per minute. The cars were initialized with a strategy following heuristics that mimicked selfish drivers, and then these strategies were improved by 
the smart vehicles using a method similar to DQNs. The underlying NN received the 18 elements of the state space as input, had a single hidden layer with 12 neurons, and 3 output units representing the desirability of each lane change (change left, maintain lane, or change right).

In the reported experiments, the cars were forced to maintain a 2 iteration headway for safety. Each episode of training lasted for 400 iterations, began with 200 cars over 3 lanes, and the cars had desired speeds determined from an $\mathrm{N}(60,8)$ distribution. At $100 \%$ selfish traffic, the cars averaged a 36.8 mean squared error between their actual and desired speed; with $95 \%$ selfish cars, and $5 \%$ smart cars, the traffic error dropped to 34.40 . At $100 \%$ smart cars, the same error further dropped to 9.66. Therefore, there is evidence that even small percentages of cooperative cars could increase the average speed of the road.

Giving each AV knowledge of the desired and current speed of all the cars on the road was critical to the design of the reward function, and it is unlikely this information would be readily available for AVs in real traffic. Furthermore, the system also had a limited amount of $\mathrm{NN}$ architectures that it could explore, considering the technology of the day. However, this paper did provide a good starting point to understand how the addition of AVs could potentially improve road speeds.

\section{Emergent Behaviors in Mixed-Autonomy Traffic}

Similar to the traffic paper involving "reducer agents" [4], reviewed in Section 2.2, the impact of modelling reducer agents using deep RL was studied in [20]. The paper formulated a mixed-autonomy traffic problem, and tested the impact of adjacent AVs in both single lane and multi-lane environments. A theoretical upper bound on average velocity for a general road was also derived, so as to measure the effectiveness of the AVs.

In the experiments, human-drivers were approximated using the IDM, and also had an additional Gaussian acceleration noise of $N(0,0.2)$. Each autonomous-vehicle consisted of a state space involving a vector of absolute velocities, positions, and lanes for each vehicle in the system, $s=\left(v_{i}, x_{i}, l_{i}\right)$, sorted in order of absolute position. The goal, as stated in the paper, was to study the behaviour of these agents rather than 
designing practical controllers, which explained the fully-observable environment as a state space. The actions that each vehicle could take involved a set of requested accelerations, $c \in\left[c_{\min }, c_{\max }\right]^{k}$, where $k$ was the number of AVs. In multi-lane scenarios, an additional vector of lane changing directions, $l \in[-1,1]^{k}$, was included, and the lane was then chosen by rounding the result. Actions $a=(c, d)$ were taken in order of absolute position of the AVs.

The paper also determined that, given $n_{a} \mathrm{AVs}$ and $n_{h} \mathrm{SVs}$, if all human-driven vehicles follow IDM, the upper bound on the average velocity of the whole system would be: $V_{n_{a}, n_{h}}=\hat{v}_{e}\left(\frac{L-n_{a} L_{v e h}}{n_{h}}\right)$, where $L$ is the total length of the ring, where $L_{v e h}$ is the length of each vehicle, and $\hat{v}_{e}\left(s_{e}\right)$ is the given equilibrium velocity, which is a function of the equilibrium headway, $s_{e}$. Conceptually, this is the same as if the AVs were removed and their length was added to one IDM-powered vehicle. In the fully human-driver setting, the upper bound is the equilibrium velocity in mixed-traffic.

The reward function used to train AVs was:

$$
r(v(t))=\max \left(0,\left\|v_{\text {des }} 1^{n}\right\|_{2}-\left\|v-v_{\text {des }} 1^{n}\right\|_{2}\right)
$$

which is an operation between the actual $(v)$ and desired $\left(v_{\text {des }}\right)$ speeds of the vehicles. This was designed to encourage a high system-level velocity, but also punished vehicles that made a decision resulting in collisions. The different scenarios experimented with included a single lane with a single AV, and both single and multiple-lanes with multiple AVs placed consecutively. The NN used was a Gaussian MLP policy with hidden layers of size [100, 50, 25], and tanh activation functions.

In the experiments run, a single $\mathrm{AV}$ appeared to keep the entire road speed near the equilibrium velocity, therefore largely reducing stop and go waves, and instead turning the cars which would stop abruptly into cars that slowed down over multiple iterations. Once multiple AVs were added, the overall velocity began to slowly approach the derived average velocity upper bound. However, both of these experiments involved a very densely packed road, so there was not much insight into how often vehicles changed lanes, if at all. Regardless, the impact of a single AV to eliminate stop and go waves is quite impressive! This paper leads to two further questions: can AVs trained with only local information that are able to share information amongst each 
other still get similar results as the single AV or multiple AVs that received the entire road, and also can an AV trained on a sparser road change lanes efficiently?

\subsubsection{AVs and Mitigating Congestion}

This subsection is focused on mitigating congestion that occurred as a result of a temporarily blocked lane, both in mixed-autonomy and fully autonomous roads. The first experiment used a state space and reward function that appeared to be effective. The second paper investigated road systems with only AVs, and therefore, similar to [20], provided an idea of what ideal traffic behaviour would appear to be like, but in this case when relieving congestion. Although this thesis does not directly consider situations where cars are completely stopped, and then suddenly released, this behaviour is similar to situations where one lane gets extremely dense traffic due to many cars suddenly entering the road, and thus, is worth investigating.

\section{Large-Scale Traffic Control Using AVs and Deep RL}

A version of centrally controlled AVs to mitigate congestion in 2-lane roads was proposed in [7]. In this paper, instead of training a network to function on cars locally and control their second-by-second decisions, a network was trained on images of the road taken from above, and the network provided cars with desired lane choices and optimal headway distances, instead of directly controlling the vehicles. The images were composed such that pixels were coloured based on whether or not they were operated by human drivers or were $\mathrm{AVs}$, and each RL agent was responsible for a specific continuous passage of the road. To coordinate the behaviours across agents without creating conflicting commands, the agents had an overlapping area of observations, which was approximately 20 percent larger than their region of control. Furthermore, the road was interpreted as a grid of pixels, further reducing the overall state-space of each image.

In this case, a single RL agent received the last 3 consecutive time steps of images, each containing a snapshot of the traffic, allowing the algorithm to approximate velocity and acceleration data without explicitly providing it. The controller was also 
limited by the number of AVs it could send commands to in a single iteration, and was designed to pass commands to the first $N_{A} \mathrm{AVs}$ on the road. The AV would then assess the safety of the recommendations from the RL agent, and adjust its speed and lane accordingly. The reward for each agent was a function of the flow of all vehicles interacting with an AV being controlled, which calculated the delay, $d_{i}$, for vehicle $i$, as the ratio between free-flow velocity and current velocity $1-v_{i} / v_{f}$, divided by the free flow speed. Therefore, an agent received a higher reward if all the vehicles that were interacting with $\mathrm{AVs}$, including the AVs, were travelling at free-flow velocity. Because the agent received a full snapshot of the road, it could determine which SVs were being impacted by the speed of the $\mathrm{AV}$, in real-time.

To create artificial congestion, one autonomous vehicle in the right lane was forced to slowly brake after 50 iterations, decelerating by $1 \mathrm{~m} / \mathrm{s}$ until stopping, and thereafter remaining stopped until just after 550 iterations. This forced all cars following the stopped car not to be able to change lanes due to fast speeds in the adjacent lane. Comparing the ability for a single agent, which had a region of control of only $1 / 4$ of the road, to a series of agents that had control of the entire road, the results showed that the series of agents resulted in less overall delay when relieving the congestion.

In road systems modelled using NS modelling, where the entire road system shares a maximum speed, it is possible, by combining snapshots of traffic, for an AV to approximate how fast or slow the adjacent vehicles are travelling relative to the maximum road speed. This paper is also one of the few that determined the effectiveness of controllers that were spread out along the road, rather than clumped together. Finally, having a reward function that is an operation of all AVs on the road is an effective way to gauge the performance of the entire road for the RL agent, and this motivated the selection of some reward functions used in the thesis (specified in Chapter 5).

\section{Cooperative Lane Changing via Deep RL}

The goal of the paper [18] was also to minimize the delay of individual vehicles between their desired and current speeds, by focusing solely on lane changing behaviour, similar to [9], discussed earlier in this section, but on roads fully populated with AVs 
that differed in their lane changing strategies.

In the road model used, each vehicle was represented by 4 cells in a grid, where each cell represented 1 meter. The cars were also trained on 3 series of frames of traffic snapshots, $M_{t}^{(i)}$, which each cover 15 meters ahead and 5 meters behind the front of the AV. The cars also received the speed difference between the current vehicles' actual and desired speeds at each of the snapshots. All vehicles therefore only relied on on-board sensors to detect surrounding vehicles, and these snapshots were represented as a binary matrix with corresponding elements equal to one if a car was on the grid cell, and zero otherwise. This was a similar road model to the continuous NS modelling scheme, briefly mentioned towards the end of Section 2.2.

The set of actions each vehicle could take were to switch left, switch right, speedup, or maintain the current speed. A collision-check process was implemented so vehicles would be automatically slowed down to avoid a collision. In this road model, first, new vehicles were added to the beginning of the road depending on the upstream inflow rate, then the driving decision from the lane changing model was given and each speed was obtained, and finally the collision-check process was implemented. The vehicles were forced to maintain a 1 cell safety gap, and take 4 iterations to change lanes.

The reward was a sum of: the vehicle efficiency which was the current speed divided by maximum possible speed, the lane change penalty which remains constant, and traffic flow rate which was a measure of the entire traffic system. The NN used had 2 convolutional and max-pooling layers to reduce the traffic snapshot matrices, and then 2 fully connected layers to incorporate the velocity difference, each with 512 neurons, finishing with an output layer. First, an experiment involving a traffic accident bottleneck with 2 different lane change penalties, 0 and 8, to enforce cooperation and non-cooperation, were considered, followed by an experiment on free-flowing traffic. In the traffic jam experiments, the model was trained for 90,000 steps and then tested, with $\alpha=0.01, \gamma=0.9, \epsilon=0.9$, a replay memory size of 2,000 samples, and a target weight update for the DQN every 500 steps. The traffic bottleneck was held constant for 50,000 iterations, where all vehicles were "stuck", and released with ratio $p$, which decreased gradually. 
In the non-cooperative jam experiment, cars repeatedly underwent stop-and-go waves, due to excessive lane changing, which lasted indefinitely. In the cooperative version, all cars were able to consistently maintain a slow speed throughout the jam; after 74,000 iterations, the jam had fully cleared. The third experiment showed that the lane-changing penalty had to increase as inflow to the road increased, and that there was a trade-off between individual and overall road travelling efficiencies.

The underlying road model for these experiments was very similar to the NSmodel, but instead of providing cars gaps, they were given snapshots of the road. Furthermore, this performance function incorporated information about all the cars on the roads' efficiency, but since they were all AVs, this information could be feasibly shared. Since every single car in the road was treated as an AV, these experiments provided an idea of how to obtain the empirical upper bound on how well mixedautonomy traffic would perform, specifically when mitigating congestion in a dense portion of the road.

\subsubsection{Single AVs Navigating Roads Safely and Efficiently}

This subsection focuses on different methods for modelling single AVs in mixedautonomy environments. The first paper compares the impact of a single AV, and a coordinated fleet of AVs, against a rule based driver. The following two papers are different examples of modelling single AVs, differentiated by the environment, state space, and network architecture.

\section{Multi-Agent Coordinated Learning for Autonomous Driving in Highways}

The paper [21] compared a single AV, and then a fleet of coordinated AVs and their impact on the average road speeds on 2-lane roads, compared to rule-based driving algorithms. In this case the state space for each $\mathrm{AV}$ was the distance and speed to each of the 4 adjacent vehicles, ahead and behind, in the current and other lane. The road consisted of a tailgating lane, which had a speed limit of $30 \mathrm{~m} / \mathrm{s}$, and an overtaking lane, which had a speed limit of $40 \mathrm{~m} / \mathrm{s}$.

The reward function was the minimum of the forward and backward reaction 
times, calculated as the difference in distance between the AV and its adjacent vehicles, and the speed of the adjacent vehicle. If the current vehicle entered within 3 meters of an adjacent vehicle, it received a reward of -5 . Therefore, safety, rather than speed, was the primary goal of these AVs.

Episodes were ended either when a collision occurred or after 400 steps. First, Tabular Q learning was compared with DQN when modelling a single AV, where the DQN modelling used a shallow NN, with 5 inputs, 6 hidden layers, and 2 outputs. In this case, DQN made a significant improvement immediately, but then took 50,000 episodes to noticeably outperform Tabular Q Learning, which required between 500 and 1000 episodes to converge. Both significantly outperformed the "expert" driver after 500 episodes.

Once all the vehicles were coordinated, rule based drivers were outperformed immediately, and the AVs obtained significantly higher rewards, suggesting that they were much safer. Furthermore, even though the reward function did not directly reward the autonomous vehicle for travelling faster, the actual average speed improved from $29.5 \mathrm{~m} / \mathrm{s}$ for rule-based drivers, to $34 \mathrm{~m} / \mathrm{s}$ for coordinated approaches, compared with roughly $31 \mathrm{~m} / \mathrm{s}$ for individual autonomous drivers.

The coordination of AVs was realistic and will be applied to this thesis, because given that they are already connected to a central system (similar to planes in airtraffic), they can also all share a network. Furthermore, it appears that Tabular Q Learning is also worth testing when the state and action space are small enough that the vehicle will be able to explore each combination adequately.

\section{Automated Speed and Lane Change Decisions Using Deep RL}

The paper [2] trained a single AV using a DQN, but compared two different underlying NN architectures. In this scenario, the state space for the vehicle being controlled involved 27 inputs, including the relative speed, position, and lane of the 8 adjacent vehicles, the AVs current speed, and two indicators to determine if the right and left lane existed to change to. The agent could choose to accelerate, decelerate, maintain speed, or brake, and whether or not to change lanes. In this first experiment, the AV was only given the ability to change lanes while the IDM controlled speeds; in 
the second, it was also given the ability to change speeds. The reward was positive and derived from the distance driven during the iteration, divided by the maximum possible distance that could have been driven. Further, if the vehicle caused a collision the episode was terminated with a reward of -10 , and if a vehicle made a decision that brought it close to causing a collision, a reward of -10 was given without terminating the episode. A reward of -1 was given for each lane change. The final results were compared to the reference lane changing model, and the reference speed and lane change model, normalized to between 0 and 1 .

The first network architecture that was tested had 2 hidden RELU layers with 512 units each. The second net had 2 convolution and max pooling layers for the relative speed, position, and lane of surrounding vehicles, and this was combined with the information about the AV using a single-fully connected layer. All the training was achieved using double DQN, with an $\epsilon$-greedy policy, where $\epsilon$ was constantly decreasing. The simulation involved a 3-lane highway, where the AV was $16.5 \mathrm{~m}$ in length, and the surrounding vehicles were $4.8 \mathrm{~m}$ in length, on a continuous roadway. The 8 surrounding vehicles were positioned in front of the agent, so that the vehicles in front were slower and vehicles behind were faster, forcing the agent to decide between overtaking and following, depending on the speed of the upcoming vehicles. Episodes that involved a collision very early due to bad road placement were deleted; otherwise, all episodes were bounded by time unless a collision occurred due to a bad decision by the AV. If the agent did not end up in a collision, the average speed of the AV during the episode determined the performance score.

Each run involved 2 million iterations for training, and then after every 50,000 iterations, the $\mathrm{AV}$ was evaluated for 1,000 random episodes. The results showed that the model training with a CNN was able to travel 10 percent faster than the IDM when allowed to control the speed of the AV, and otherwise, travelled slightly faster than the IDM when only allowed to control the lane selection. This only took between 50,000 and 100,000 iterations of training as well! Conversely, the fully connected NN reached close to the reference speed when only accounting for lane changes, and was not able to reach the reference speed even within 300,000 iterations when the AV had to control its own speed, because of the high number of collisions faced. 
Interestingly, it appeared that the $\mathrm{CNN}$ architecture received a high reward relatively quickly because the agent almost never chose to change lanes in the beginning. This appeared to be so that it could understand which patterns allowed for safe lane changes, and once this was learned began changing lanes to travel faster without losing reward due to collisions, finishing with a choice to change lanes around one percent of the time. Therefore, this paper demonstrated how effective a CNN, which can translate patterns to new situations, is, both at determining collision-avoiding actions and for quickly training an agent to behave in a collision-free manner. However, it is worth exploring whether restricting the action space of the AV to be collisionfree from each state could potentially improve the performance of the Feed-Forward network, which is slightly more efficient computationally. It also motivated the idea of investigating road dynamics when $\mathrm{AVs}$ that do not change lanes share the road with human drivers that do.

\section{Autonomous Highway Driving using DRL}

The paper [12] also experimented with training a single AV to navigate a highway setting, and tested the method on a road of varying densities. The AV was trained using Double DQN, but used a cleverly-selected feature set, which included a prioritized experience replay buffer that stored all decisions made by a vehicle that would result in a collision.

The AV being controlled could be surrounded by at most 6 vehicles in the 3lane highway, which referred to the vehicles ahead and behind on its current and all adjacent lanes. Furthermore, with such a model, the vehicle could be at different positions within a lane. Therefore, the full state space was the relative distance and velocity both in terms of latitude and longitude on all adjacent lanes. The decisions a vehicle could make were to maintain current conditions, accelerate, brake, hard brake, or change lanes. Changing lanes required five iterations of time, which gave the vehicle an opportunity to abort the lane change. The reward function was composed of a combination of the difference between the AVs current and desired speeds, the desired lane relative to the current lane, and the relative distance to the car ahead based on velocity. 
In the experiment conducted, up to 30 cars were placed within a distance of 250 meters of the AV, and the vehicles' actions translated to an acceleration or brake of $2 \mathrm{~m} / \mathrm{s}$, and a hard brake of $4 \mathrm{~m} / \mathrm{s}$. The desired velocity was $30 \mathrm{~m} / \mathrm{s}$, the desired lane position was the center lane, and the desired safe distance was $40 \mathrm{~m}$, leaving a 1.33 second buffer to react to avoid a collision. The AVs controller was evaluated every 100 episodes: it required 2,000 episodes to converge, and 10,000 episodes of training in total, where each episode lasted 200 samples or until a collision occurred. The associated DNN had 2 hidden layers each with 100 hidden units.

Overall, the AV outperformed the IDM combined with the SUMO platform for lane changes, until the density of the road increased to 20 cars per lane per km. In summary, this paper provided interesting insights into lane changing, which ultimately were not applied in this thesis, but are interesting to consider for future work.

\subsection{Conclusions}

This chapter began by reviewing previous work on modelling road systems used to simulate human drivers and the resulting traffic jam dynamics that occur. This thesis made use of micro-traffic modelling, inspired by the NS system, but allowing for cars to behave more aggressively, both for single and multi lane roads.

Next, methods for performing RL without an underlying model of the environment, in environments with large state spaces, was covered. Model-free RL is used in this thesis, ranging from Tabular Q Learning to DQNs.

Finally, past work where RL was applied to AVs in order to navigate road traffic was reviewed. First, previous works were analyzed, which establish that on mixed autonomy roads, a small percentage of AVs can improve overall speeds on the road. The reward function for these AVs involved knowing the speed of every single car on the road, which is not a luxury given to AVs in this thesis. Next, prior work involving analyzing how AVs impact the ability of cars to clear up congestion due to a blocked lane, were summarised. The state spaces for all AVs in these works were three consecutive snapshots of surrounding environment of the AVs, which allowed for the underlying function approximator to see partially long term consequences of 
actions. The reward functions used were only a product of the state space of the AVs on the road, which also motivated the approach that was used in this thesis. Finally, papers modelling single AVs to navigate through obstacles on the road were explored. In these works, the primary takeaways were the different architectures of $\mathrm{NN}$ that worked, and that starting with tabular reinforcement learning is important because even the most basic Q Learning can still perform well given a small enough state space.

The next chapter describes the road traffic model and the criteria specifically used in this thesis to run and assess experiments. After that, in Chapter 4, the model for simulating human drivers and the associated simulation results are given to create benchmarks for road conditions. Finally, Chapter 5 will cover the methods that were used to train AVs, and determines how they are able to improve traffic dynamics, specifically when it comes to preventing the formation of emergent jams. 


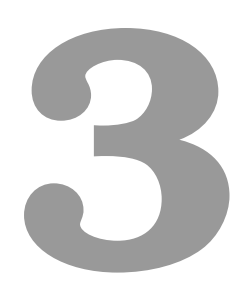

\section{Traffic Representations}

\subsection{Introduction}

The goal of this chapter is to introduce the micro-traffic modelling framework used simulate road systems, and to state the criteria that will be used to evaluate all of the experiments in this thesis. This chapter begins with a description of the abstract road and car representations, including the characteristics that define each traffic experiment, in Section 3.2. This is followed by presenting algorithms used to maintain the road representation, in Section 3.3. Finally the criteria used to evaluate any traffic experiment, is covered in Section 3.4. 


\subsection{Traffic Representations}

The traffic experiments analyzed in this thesis are conducted using micro-traffic models, implying that all cars independently determine and execute lane and speed decisions during every time interval. These decisions are made by calculating the gaps between each car and its neighbouring cars, comparing these gaps to the current speed of the car, and then stochastically accelerating or decelerating with the goal of travelling as fast as possible in the most optimal lane. All experiments are enforced to be collision free, restricting the decisions each car makes, such that by the end of the iteration, no two cars will have travelled to the exact same road cell. To enforce these constraints and model the traffic system, the road is represented as a tensor and the cars are represented by a matrix. In each iteration, both the road tensor and car matrix are updated based on the decisions that each car makes. In this section, the road tensor and car matrix representations are given, followed by an overview of the variations that will characterize each traffic experiment.

\subsubsection{Road Representation}

Roads are divided into cells, and the first and last cell are connected so that the road forms a loop, effectively representing an infinitely long highway stretch with periodic boundary conditions. For the example road depicted in Figure 3.1, this means that cell 1 follows cell 15. At most a single car can occupy any given cell at one time, no cars can be in between two adjacent cells, and each cell represents the same distance (i.e, meters on the road) regardless of its specific location in the loop.

R1: Road With 15 Cells, 3 Lanes, and 8 Cars, MaxSpeed 6, No Lane Preference

\begin{tabular}{|l|l|l|l|l|l|l|l|l|l|l|l|l|l|l|l|}
\hline & $\mathbf{1}$ & $\mathbf{2}$ & $\mathbf{3}$ & $\mathbf{4}$ & $\mathbf{5}$ & $\mathbf{6}$ & $\mathbf{7}$ & $\mathbf{8}$ & $\mathbf{9}$ & $\mathbf{1 0}$ & $\mathbf{1 1}$ & $\mathbf{1 2}$ & $\mathbf{1 3}$ & $\mathbf{1 4}$ & $\mathbf{1 5}$ \\
\hline $\mathbf{L 3}$ & $c_{1}$ & & & $c_{2}$ & & & & & & & & & & $c_{3}$ & \\
\hline $\mathbf{L 2}$ & & & & & $c_{4}$ & & $c_{5}$ & & & & & & $c_{6}$ & & \\
\hline $\mathbf{L 1}$ & & $c_{7}$ & & & & & & & & & $c_{8}$ & & & & \\
\hline
\end{tabular}

Figure 3.1: Example of a 3-lane road. 
The road is represented as a 3D tensor. The rows correspond to each lane, $i$, and the columns correspond to each cell on the road, $j$. The speed of a car represents how many cells forward the car will move in the same lane, and cars can only change to adjacent lanes. The depth of each [row, column] pair contains features for the associated road cell, which include the:

1. Front Gap $(f G)$ : The number of cells between the current cell and the cell that the next car is on (in the same lane), excluding the current cell. This takes a minimum value of one if there is a car on the next cell in the same lane, and a maximum value equivalent to the number of cells in the lane.

2. Back Gap $(b G)$ : The number of cells between the current cell and the previous cell that a car is on, in the same lane, including the current cell. This takes a minimum value of zero if there is a car on the current cell, and a maximum value equivalent to the number of cells in the lane (the road length).

3. Maximum Speed $(m S)$ : The maximum allowed speed of the current road cell. Typically, this remains constant on the entire road, but in some special road conditions, a particular cell (or group of nearby cells) can have a purposefully lower maximum speed, so as to simulate a random slowdown, a construction zone, or a temporarily blocked lane.

4. Cell Preference $(p)$ : The preference (from -1 to 1 ) compared to the same cell in each lane on the road, where a higher preference represents a higher chance that a car will choose that particular lane if a safe lane change is available. This preference value can represent driving lanes and passing lanes, and negative preference values represent an entry $(-1)$, an exit $(-0.75)$, or an upcoming entry or exit $(-0.5)$. The use of preference to model entries and exits will be explained further in Section 3.3, and the model for lane decisions will be explained further in Section 4.2 .

The road object also stores (as constants): the total number of cells in one lane $\left(R_{\text {len }}\right)$, the total number of lanes $\left(R_{\text {lanes }}\right)$, the maximum speed of all cells in the road 
$\left(R_{\mathrm{mSp}}\right)$, the number of cars on the road at the start of the experiment $\left(R_{\mathrm{nC}}\right)$, the distribution of each car type ( $R_{\text {types }}$, explained in the next section), the reciprocal of the number of iterations after which a car is expected to exit the road after entering $\left(R_{\text {exit }}\right)$, and the number of iterations after which some road cells have their maximum speeds reduced to zero for one iteration to model random stopping $\left(R_{r p}\right)$.

In what follows, the following notation used to access any road cells feature $(f)$, will be $R_{i, j}(f)$, where $i$ and $j$ correspond to the lane and cell. For example, the notation and values for the Front and Back Gap of cell 5 in lane 2 in the road shown in Figure 3.1 (the cell which has the car labelled $c_{4}$ ), is $R_{2,5}(f G)=2$, because $c_{5}$ is two cells ahead in the same lane. Similarly, $R_{2,5}(b G)=0$, because, by definition, there is a car on this cell. For the previous block on the same lane, block 4 in lane 2, $R_{2,4}(f G)=1$ and $R_{2,4}(b G)=6$.

\subsubsection{Car Representation}

At the start of each iteration, each car is on a unique road cell, and is represented by a vector containing the following elements:

1. ID (id): Unique ID for the car so that it can be traced during the experiment.

2. Lane (i): The lane (row) of the road that the car is on.

3. Place $(j)$ : The cell (column) of the road that the car is on.

4. Speed $(s)$ : The speed at which the car is travelling.

5. Type $(t)$ : Type of car (normal, passive, aggressive, or autonomous), which impacts how it makes its speed and lane decisions (the details of the different types are given in Section 3.2.3).

6. Exit Factor (ef): In an open road setting, this represents the likelihood that a car will be exiting in the upcoming iterations. This value increases at every iteration, following a normal distribution with a mean and standard deviation specified by the $R_{\text {exit }}$ value of the road. This impacts lane decisions, as explained further in Section 4.2. 
All car vectors are maintained as rows in a matrix which is sorted by place and lane. In the algorithms, cars are referenced by their IDs. For example, feature $f$ for a car with ID 1 is accessed with the notation $c_{1}(f)$. Throughout the experiments, all the features change for each car on every iteration except the ID. The road is able to identify the location of the cars by matching the Lane and Place fields.

\subsubsection{Characteristics of Traffic Environments}

Traffic experiments are categorized by the type of simulation, the type of road, the density, and types of cars. These parameters affect the optimal conditions of the road, and consequently influence the amount of traffic jams that occur.

\section{Simulation Categories}

In any experiment the simulation belongs to one of two categories:

1. Closed Roads: No cars enter or exit the road.

2. Open Roads: Cars are able to enter and exit the road from designated cells in the first lane, specified by the cells preference feature.

\section{Types of Roads}

There are three types of roads characterized by the number and properties of lanes:

1. Single Lane: Single lane roads involve only speed decisions and no lane decisions.

2. Double Lane: Double lane roads involve both speed and lane decisions, as there is the option of changing lanes.

3. $n$-Lane: $n$-Lane roads have more than two lanes. These roads are not evaluated with autonomous vehicles in the thesis, but were run with "human-drivers" to understand how the road dynamics generally change as more lanes are added.

Both road type and simulation category are specified when the initial road for the experiment is constructed, along with the associated road constants. 


\section{Types of Cars}

Cars are also of different types, which are classified into the following categories:

1. 0 (Normal): These cars accelerate and decelerate with a probability proportional to the gap in front of them, and change lanes proportionally with the lane preference.

2. 1 (Aggressive): These cars accelerate quickly but also brake early and aggressively. They behave in a greedy manner, with many overreactions. They also prefer the passing lane regardless of the lane preferences.

3. -1 (Passive): These cars have a low probability of accelerating unless the gap in front of them is very large, and have a very high probability of decelerating early, therefore limiting the number of abrupt brakes. They also prefer to maintain their current lane unless the gap in an adjacent lane is significantly larger.

4. 2 (Autonomous): These cars make decisions based on their own reinforcement learning model, and are explored in Chapter 5.

The numbers of each type of car are specified when the car matrix is constructed at the start of the experiment. If a new car enters the road, the type is randomly assigned using the same ratio of passive, aggressive, and normal cars that the road began with. Cars enter in the first lane with a speed that is uniformly sampled from all speeds less than the max speed, and at a cell sampled from the available cells designated for entry.

\section{$3.3 \quad$ Road Updates}

The primary function of the road representation is to assist cars in determining their gaps with adjacent cars, and managing the entry and exit of cars on open roads. In each iteration, the road first manages the entries of new cars, and then updates all gaps. After this, each car makes a lane and speed decision, based on logic explained in Chapter 4 and Chapter 5. Finally, some cars will exit the road. 


\subsubsection{New Car Entry}

Cars enter the road onto designated cells in the first lane, specified when the road is initially constructed, using Algorithm 1: car-entry, which has the following steps:

1. The entry cells available for the road are found by filtering the cells in lane 1 with a preference value -1 , and with no car currently on that cell. The actual cell numbers are stored in $E$, and the number of cells available to enter on are stored in $n_{E}$.

2. The total number of cars that will be permitted to enter the road system is calculated as:

$$
\min \left(n_{E}, \max \left(\operatorname{round}\left(\mathrm{N}\left(R_{n C}-n_{\text {cur }}, 0.5\left(R_{n C}-n_{\text {cur }}\right)\right)\right), 0\right)\right)
$$

where $n_{\text {cur }}$ is the current number of cars, $R_{n C}$ is the initial number of cars stored in the road constants. This implies that the number of cars entering the road will be randomly selected using a normal distribution with a mean that is the difference between the initial and current number of cars and a high variance.

3. Entering cars are assigned starting values using the same distributions that were used to initialize the road: each car is assigned a unique ID, the type is sampled from the same type ratio used at the start of the experiment, the speeds are sampled uniformly between unity and the max speed, and the places are sampled from the available designated entry cells.

\subsubsection{Road Gap Updating Algorithm}

Updating gaps is done on each lane (i) independently, using Algorithm 2: update-road-gaps, via the following steps:

1. Cars belonging to lane $\mathrm{i}$ are selected from the car matrix $\mathrm{C}$, sorted in an increasing order by place value, and stored in a new matrix Ct. 
Algorithm 1 car-entry $\left(R 1, R_{c}, n_{c u r}, I D_{\max }\right)$ : Entering Cars

\section{Input:}

i) The first lane of the road tensor $R 1$

ii) The road constants for number of cars initially on the road, the max speed of the road, and the initial type distribution of cars: $R_{n C}, R_{m S p}, R_{\text {types }}$

iii) The number of cars currently on the road $\left(n_{\text {cur }}\right)$, and the indices of the cars in lane 1 , ind

iv) The max ID of the existing cars $I D_{\max }$

\section{Method:}

1: $E=$ filter $\left(R 1_{1,:}(p)=-1, R 1_{1,:}(j) \neq i n d\right)(j)$ \{determine entry cells

2: $n E=\min \left(\right.$ length $\left.(E), \max \left(\operatorname{round}\left(N\left(R_{n C}-n_{\text {cur }}, 0.5\left(R_{n C}-n_{\text {cur }}\right)\right)\right), 0\right)\right)$

3: if $n E==0$ then

4: $\quad$ end

5: else

6: $\quad$ data.frame(

$I D=\left(I D_{\max }+1\right):\left(I D_{\max }+n E\right)$,

$j=\operatorname{sample}($ num $=n E$, vector $=$ entries $)$,

$i=1$,

$s=\operatorname{sample}\left(\right.$ num $=n E$, vector $\left.=\left\{1: R_{m S p}\right\}\right)$,

$t=\operatorname{sample}\left(\right.$ num $=n E$, vector $=\mathrm{c}(-1,0,1)$, weights $\left.=\left[R_{\text {types }}\right]\right)$,

ef $\left.=\operatorname{repeat}\left(N\left(R_{\text {exit }}, R_{\text {exit }}\right), n E\right)\right)$

\section{7: end if}


2. (a) If there are no cars on the selected lane, meaning Ct is empty, then all forward and backward gaps are set as equal to the road length and the algorithm terminates.

(b) Else, two variables (prev and next) are initialized as the last car (car with highest cell value) and first car (car with the lowest cell value), respectively.

3. Loop over all road cell numbers in the lane in an increasing order $\left(x \in 1: R_{l e n}\right)$ :

(a) If the cell value of next $\left(\mathrm{Ct}_{\text {next }}(j)\right)$ is equal to the $\mathrm{x}$, then set prev $=$ next, and set next to the next car in Ct. If next is already the last car in the car matrix, then next becomes the first car again (since the cars are in a loop). Else, maintain the current prev and next values.

(b) The current cells Back Gap is assigned the difference between the current cell and place of the car currently in prev: $\mathrm{R}_{i, x}(b G)=\mathrm{x}-\mathrm{Ct}_{\text {prev }}(j)$.

(c) The current cells Front Gap is assigned the difference between the place of the car currently in next and the current cell: $\mathrm{R}_{i, x}(f G)=\mathrm{Ct}_{\text {next }}(j)-\mathrm{x}$

In summary, the gaps are updated by subtracting from the latest and next car on the road by traversing the road and updating placeholders for cars as they are passed. Therefore, in each iteration, updating the road requires two computations and two memory accesses for each road cell, and one access of all the features of every car.

\subsubsection{Exiting Cars}

On every iteration, the exit probability for each car on the road is independently increased by a random variable with a mean and standard deviation, given by $R_{e x i t}$ : $c_{i}(e f) \leftarrow c_{i}(e f)+N\left(R_{e x i t}, R_{e x i t}\right)$, where $i$ is the ID of the car being updated, and $R_{e x i t}$ is the inverse of the number of iterations, on average, after which a car will be in a state to exit the road. The reason for making this decision stochastically is that sometimes drivers end up in an exiting lane without intending to, and cannot change lanes out of that lane. At the end of each iteration, any cars in the first lane at the specified exit cells on the road are stochastically removed with a probability specified from their exit factor: $\min \left(1, c_{i}(e)\right)$. This is executed using Algorithm 3: car-exit. 

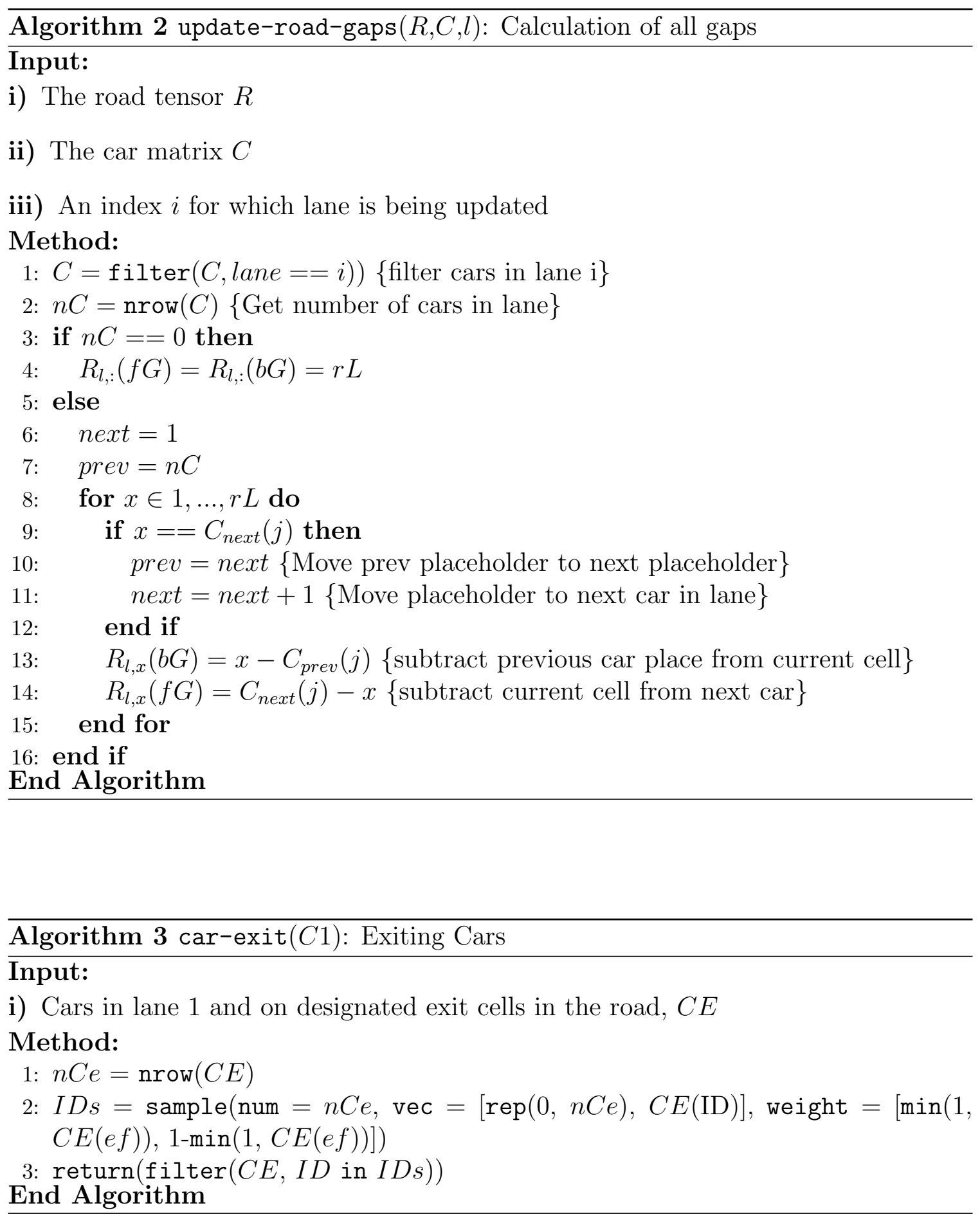


\subsection{Experiment Assessment Criteria}

To evaluate how optimally a road system is moving, the following criteria, calculated at the end of every iteration, are proposed:

1. Number of Cars: The total number of cars on the road.

2. Average Road Speed: The mean of all car speeds on the road.

3. Absolute Sum of Speed Changes: The mean of the sum of absolute differences between starting and finishing speed for all cars that do not exit the road.

4. Number of Lane Changes: The number of cars that changed lanes.

5. Percentage of Optimum Cars: The percentage of cars travelling at the optimum speed, which is calculated as equal to the minimum of the maximum road speed and the inverse of the road density.

6. Percentage of Stopped Cars: The percentage of cars travelling at speed 0.

7. Percentage of Jammed Cars: The percentage of cars travelling with speed less than the optimum speed.

8. Percentage of Grouped Jammed Cars: The percentage of cars belonging to a group, where 5 or more consecutive cars on the same lane have a Front Gap less than the road density.

9. Percentage of Aggressive Cars: The percentage of cars travelling faster than the optimum speed of the road.

10. Lane Distribution: The percentage of cars in the first lane.

The primary goal for any traffic system is that the average road speed (Criterion 2 ) should be as close to the theoretically maximum speed of the road as possible. The secondary goal for any road system is to minimize the absolute sum of speed changes (Criterion 3) and minimize the percentage of fully stopped cars (Criterion 6 ), because frequently changing speeds and stopping creates a higher probability 
of forcing cars behind to overreact in order to avoid collisions, therefore creating emergent traffic jams. The tertiary goals are to ensure that the different percentages of jammed cars and aggressive cars stay low and decrease quickly in the case of unforeseen circumstances which artificially spikes these metrics, such as perturbations or enforced slowdowns.

\subsection{Conclusions}

In this chapter, the road and car representations were given, along with the logic for all the different road features that can impact optimum road speed and therefore influence traffic flow. Furthermore, the primary, secondary, and tertiary criteria used to evaluate traffic conditions were stated, and will be used to evaluate experiments going forward.

The next chapter will cover the algorithms used for Stochastic Vehicles (SVs), which simulate human drivers, and also the experimental results for a variety of roads, all of which will only contain SVs. The benchmarks from the experiments run in Chapter 4 will be used in Chapter 5, to evaluate whether replacing the Stochastic Vehicles with different percentages of autonomous vehicles can objectively improve the traffic criteria that were stated above in Section 3.4. 


\section{4}

Stochastic Traffic Patterns

\subsection{Introduction}

Cars are either categorized as Stochastic Vehicles (SVs), meaning they simulate human drivers by making decisions based on a set of rules which incorporate randomness, or Autonomous Vehicles (AVs), meaning they make decisions based on an underlying reinforcement learning model. On each iteration, cars must determine what speed to travel at, and which lane to travel on, including whether or not they should exit the road. To make these decisions, cars only receive their own features (speed, lane, place, exit probability), and features of the road cell on which they start the iteration on (the Front and Back Gaps, Maximum Speed, and Preference Factor). This resembles what is visible to a human driver, or sensed by the LIDAR/cameras used by an AV, in real driving scenarios.

This chapter focuses specifically on the algorithms used for modelling SVs, but 
many of the constraints incorporated in these algorithms, to enforce collision free decisions, also apply to AVs. The algorithms used for SVs to make lane and speed decisions are given in Section 4.2, followed by an analysis of the corresponding experiments in Section 4.3. Finally, a brief summary of the main results is given in Section 4.4, and conclusions are given in Section 4.5.

\subsection{Car Updating}

On every iteration, cars are aware of their own speed and lane, and use the road as a reference to obtain gaps to their neighbouring cars. These gaps determine the maximum speed that they can choose during the iteration, so as to ensure that they will not create a collision. Specifically, a car must choose a speed that is less than the front gap between itself and the car ahead at the start of the iteration, in the lane that it will choose during that iteration. This section covers specifically how the car chooses which lane to travel on, and accordingly, how the car determines what speed to travel at.

\subsubsection{Lane Decisions}

Cars make lane decisions stochastically, and these decisions are influenced by the gap available in each lane onto which they can change. A right lane change refers numerically to going down one lane in value, and alternatively, a left lane change refers to going up one lane. Because cars are only aware of their gaps to adjacent cars and not of the speed of these adjacent cars, to ensure that no collisions occur, the following constraints must be satisfied for a lane change to be valid:

1. The lane onto which the change is being made must exist. For example, in Figure 4.1, a car in lane L1 cannot make a right lane change, since lane L1 is the rightmost lane.

2. A car cannot change lanes and also finish the iteration with speed 0 . This is because, according to the next constraint (Constraint 3), cars cannot decelerate 
R2: MaxSpeed 5, Colour show available blocks for each car depending on current speeds

\begin{tabular}{|l|l|l|l|l|l|l|l|l|l|l|l|l|l|l|l|}
\hline & $\mathbf{1}$ & $\mathbf{2}$ & $\mathbf{3}$ & $\mathbf{4}$ & $\mathbf{5}$ & $\mathbf{6}$ & $\mathbf{7}$ & $\mathbf{8}$ & $\mathbf{9}$ & $\mathbf{1 0}$ & $\mathbf{1 1}$ & $\mathbf{1 2}$ & $\mathbf{1 3}$ & $\mathbf{1 4}$ & $\mathbf{1 5}$ \\
\hline L3 & & & & $c_{1}$ & $c_{2}$ & & & & & & & & & $c_{3}$ & \\
\hline L2 & & & & & $c_{4}$ & & & $c_{5}$ & & & & $c_{6}$ & & & \\
\hline $\mathbf{L 1}$ & & $c_{7}$ & & & & & & & & & & & $c_{8}$ & & \\
\hline
\end{tabular}

Figure 4.1: An example of a 3-Lane Road, where coloured regions represent the available cells for each vehicle. Notice that no two vehicles are able to travel to the same cell, and therefore a collision will not occur, due to the lane-change and speed constraints.

when changing lanes, and it is impractical to change lanes while remaining stopped. For example, in Figure 4.1, $c_{1}$ cannot change from L3 to L2, from cell $R_{3,4}$ to $R_{2,4}$.

3. The car must maintain its current speed or accelerate when changing lanes. Furthermore, the gap used to determine at what speed it is feasible to travel in the new lane is the minimum of the Front Gap of the lane being changed to, and the Front Gap of the current lane plus one. This prevents any chance of a collision if both the current car and the car ahead in the same lane choose to change lanes on the same iteration, but the car ahead changes at a slower speed. The following are some examples of how this constraint prevents collisions, using Figure 4.1:

(a) If $c_{5}$ is travelling at a speed greater than 4 , it cannot change lanes in the present iteration. This is because the front gap for $c_{5}$ in the right lane (L1) is 5, meaning the maximum speed $c_{5}$ can take in L1 is 4 , and this would result in decelerating and changing lanes during the same iteration. Furthermore, the left gap in L3 is 6 , but because $c_{5}$ does not receive the speed of $c_{6}$, it is theoretically possible for $c_{6}$ to also change to L3 into cell 13 , which prevents the left lane change for $c_{5}$.

(b) If $c_{4}$ is making a right lane change, the maximum speed it can travel at is 
3 , because nothing prohibits $c_{5}$ from also changing lanes at the same time with a speed of 1 , and this would result in a collision if $c_{4}$ is travelling faster.

4. The car that changes lanes must travel to a cell at the end of the iteration that the car behind, in the new lane, cannot travel to. This means that the maximum speed of the road must be strictly bounded above by than the Back Gap from the current cell in the new lane added to the current speed of the car (i):

$$
R_{m S p}<R_{c_{i}(\text { newLane }), c_{i}(\text { cell })}(b G)+c_{i}(\text { speed })
$$

This follows a similar logic as multi-lane NS modelling, but allows for cars to be more aggressive [14]. Some examples from Figure 4.1 are:

(a) Car $c_{5}$ can only change lanes to the left from L2 to L3 if the car is currently travelling at least at speed 3; it will not decelerate and change lanes at the same time because of Constraint 3. Otherwise, assuming car $c_{2}$ accelerates to the max speed, 5 , on the same iteration that car $c_{5}$ changes with a speed less than 3 , there will be a collision.

(b) Car $c_{8}$ cannot make a left lane change, because based on Constraint 3, the maximum speed it could travel at on L2 is 4, and therefore it will potentially collide with $c_{6}$, and accordingly violate Constraint 4 :

$$
R_{m S p}=R_{c_{8}(i+1), c_{8}(j)}(b G)+c_{8}(s) ; R_{m S p}=5 ; R_{c_{8}(i+1), c_{8}(j)}(b G)=1 ; c_{8}(s) \leq 4
$$

5. If there is no car in the lane immediately adjacent, but there is a car two lanes adjacent, such that if the car two lanes adjacent was in the lane immediately adjacent on the same road cell, it would violate Constraint 4, then the car in the lane further from the exit/entry lane gets priority to make the lane change change. An example of this can be seen in Figure 4.1, between $c_{3}$ and $c_{7}$ : both cars are could technically change to cells 3 and $\mathbf{4}$ in L2, and therefore, because $c_{3}$ is farther from $\mathrm{L} 1$, it is able to make the lane change. 
Algorithm 4: car-lane-decision, determines on which lane the car will travel and what gap to use in order to make a speed decision with. This algorithm is applied to all cars independently, receiving as inputs: the maximum allowed speed of the current road cell, the Front and Back gaps in the current and all adjacent lanes, and also all the relevant information of the car being updated at the start of the iteration (lane, place, speed, type, exit). It applies the following logic:

1. Determine the Front Gap in the current lane: $R_{\text {lane,place }}(f G)$.

2. Calculate the right lane gap, by multiplying the minimum of the Front Gap in the right lane, $R_{\text {lane-1,place }}(f G)$, and the maximum speed in the current cell, $R_{\text {lane,place }}(m S)$, with the following Boolean expressions, each of which will equal zero if the associated constraint stated above is not met:

(a) The lane inputted is not the first lane: (lane! =1).

(b) The Front Gap in the right lane is greater than one: $\left(R_{\text {lane-1,place }}(f G)>1\right)$.

(c) The speed inputted is strictly less than the minimum of the Front Gap in the right lane, and one more than the front gap in the current lane: $\left(\right.$ speed $\left.<\min \left(R_{\text {lane-1,place }}(f G), f G+1\right)\right)$.

(d) The speed inputted added to the Back Gap of the right lane is strictly greater than the maximum speed of the whole road:

$\left(\max (R:\right.$, place $(m S p))<R_{\text {lane-1,place }}(b G)+$ speed $)$.

(e) Apply 2(d) for cars two lanes away to ensure there are no conflicts and assign priority to cars in farther away lanes from the entry/exit lane.

3. Apply Step 2, above, to calculate the left lane gap, replacing 2(a) with "the lane inputted is not the last lane", and all instances of lane -1 with lane +1 .

4. Multiply all gaps by a preference factor, calculated as the sum of one and:

(a) The preference value of the lane: $R_{\text {laneX,place }}(p)$.

(b) The exit probability for the Front Gap in the current lane, and 2 times the exit probability for the car for choosing a right lane change: $\left[0, R_{\text {lane,place }}(e)\right.$, 
$\left.2 R_{\text {lane,place }}(e)\right]$. However, if there is an exit, since the preference value of the road cell will be -1 , cars with low exit probabilities will be encouraged to change out of the first lane, provided a safe lane change exists.

5. The lane selected is a sample of the up to three available lane-decisions, taken with weights calculated in the previous step, and the output of the algorithm is the minimum of the Front Gap of the lane chosen, and the Front Gap in the original lane plus one cell.

In summary, these lane decisions are designed such that cars only change lanes if there is a larger Front Gap in an adjacent lane, to make room for cars that will be entering the road, or to avoid exiting the road. Furthermore, the weight used to determine which lane is the most suitable is the actual Front Gap, which is different than the usable gap for making speed decisions. This allows for cars to change to a lane that may not have an immediate benefit, but appears to have more headway to benefit the upcoming iterations.

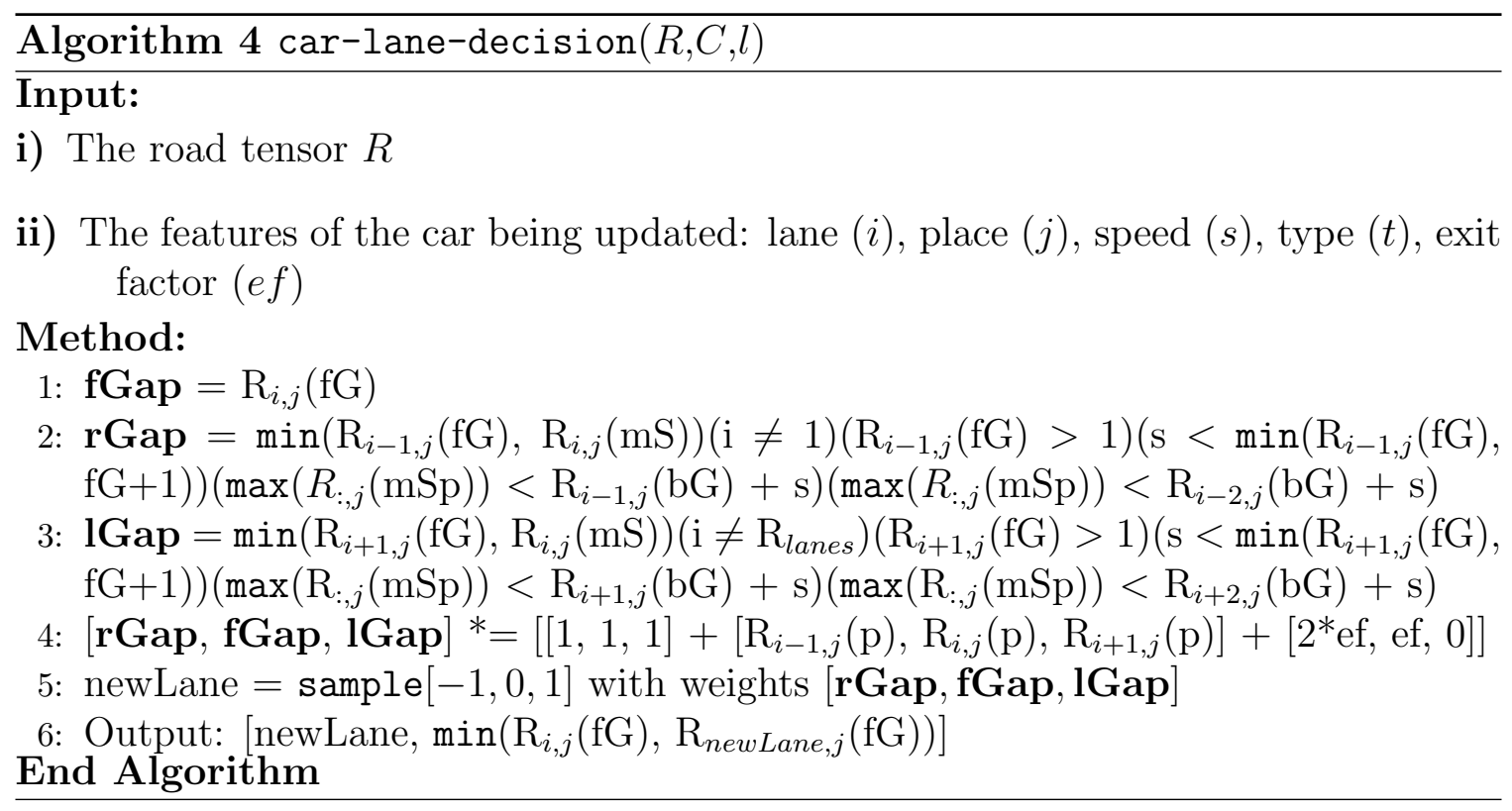




\subsubsection{Speed Decisions}

The lane decision returns a "usable gap", representing the maximum speed the car can travel on the upcoming iteration. The main difference in speed decisions between the model used for stochastic cars in this thesis, and the NS single-lane models [10], is that cars sample from any speed that is less than the minimum of the Front Gap and maximum speed, instead of just accelerating by one unit, or decelerating to either zero or exactly the Front Gap. This allows for cars to behave more aggressively, with a wider array of actions, and therefore more realistically mimicking human drivers.

The speed decision algorithm works on each car independently, and uses the usable gap determined by the lane algorithm, the type of car, and the current speed of the car, to determine the new speed of the car. This is done using the following steps (also in Algorithm 5: speed-update):

1. If the speed of a car on a cell is strictly less than the Front Gap of that cell, the car accelerates to one of the following speeds, sampled with the associated weights depending on the type of car:

(a) Normal: Sample from [speed, ..., min(maxSpeed, Front Gap - 1)] with weights $[\mathrm{sp}, \max$ Speed, .... sp +1$]$. Note that if the speed is exactly one less than the Front Gap, the car will maintain its current speed.

(b) Aggressive: Sample from [speed, ..., min (maxSpeed, Front Gap - 1)] with weights $[\mathrm{sp}, \ldots$, maxSpeed]. This means the car is likely to move as fast as possible, if there is space.

(c) Passive: Sample from [speed, min (speed + 1, maxSpeed, Front Gap - 1)] with weights [1, Front Gap - speed]. This means the car accelerates slowly and less frequently until the Front Gap is large enough.

2. If the speed is greater than or equal to the Front Gap, the car decelerates to one of the following speeds, sampled with the associated weights depending on the type of car:

(a) Normal: Sample from $[0, \ldots, \max ($ Front Gap - 1, 0)] with weights $[1,2, \ldots$, Front Gap-1, Front Gap]. This means the car has a higher 
Algorithm 5 update-speed $(C, i d$, lane, $f G, m S p)$

\section{Input:}

i) The car matrix $C$

ii) An index $i d$ for which car is being updated

iii) The new lane lane that the car being updated will move to

iv) The new front gap $f G$ in the lane the car will use to make the next speed decision

v) The maximum speed $m S p$ of the whole road

vi) The sample function is sample([listof elements], $w)$, where $w$ stands for weights, samples one of the elements in listofelements using the weights

\section{Method:}

1: $C_{i d}(i)=$ lane $\{$ Update lane for car $\}$

2: if $C_{i d}(s)<f G$ then

3: $\quad$ if $C_{i d}(t)==0$ then

4: $\quad C_{i d}(s)=\operatorname{sample}\left(\left[c_{i d}(s), \ldots, \min (m S p, f G-1)\right], \mathrm{w}=[s p, m S p, \ldots, s p+1]\right)$

5: $\quad$ else if $C_{i d}(t)==-1$ then

6: $\quad C_{i d}(s)=\operatorname{sample}\left(\left[c_{i d}(s), \ldots, \min (m S p, f G-1)\right], \mathrm{w}=[s p, \ldots, m S p]\right)$

7: $\quad$ else if $C_{i d}(t)==1$ then

8: $\quad C_{i d}(s)=\operatorname{sample}\left(\left[C_{i d}(s), \min \left(C_{i d}(s)+1, m S p, f G-1\right)\right], \mathrm{w}=\left[1, f G-C_{i d}(s)\right]\right)$

9: $\quad$ end if

10: else

11: $\quad$ if $C_{i d}(t)==0$ then

12: $\quad C_{i d}(s)=\operatorname{sample}([0, \ldots, \max (f G-1,0)], \mathrm{w}=[1, \ldots, f G])$

13: $\quad$ else if $C_{i d}(t)==-1$ then

14: $\quad C_{i d}(s)=\operatorname{sample}\left([0,1, \max (f G-2,1)], \mathrm{w}=\left[C_{i d}(s)-f G, \frac{C_{i d}(s)}{2}, \frac{C_{i d}(s)}{2}\right]\right)$

15: $\quad$ else if $C_{i d}(t)==1$ then

16: $\quad C_{i d}(s)=\operatorname{sample}\left([0, f G-2, f G-1], \mathrm{w}=\left[C_{i d}(s)-f G, \frac{C_{i d}(s)}{2}, \frac{C_{i d}(s)}{4}\right]\right)$

17: end if

18: end if

End Algorithm 
proportional chance of decelerating to the Front Gap, but can technically decelerate to 0 .

(b) Aggressive: Sample from [0, $\max ($ Front Gap - 2, 0), $\max$ (Front Gap-1, 0)] with weights [speed - Front Gap, speed/2, speed/2]. This means that the car is proportionally likely to decelerate as little as possible unless the new Front Gap is less than half the current speed, in which case the car will sharply brake.

(c) Passive: Sample from [0, 1, $\max$ (Front Gap - 2, 1)] with weights [speed - Front Gap, speed/2, speed/4]. This means that the car will tend to slow down ahead of time so it does not have to apply a sudden brake.

In summary, these speed decisions address the fact that it is very impractical for a human driver to be able to change speeds perfectly proportionally to the Front Gaps on all time instances. This rule-set significantly decreases the likelihood that a car will fully brake but also does not allow cars to maintain unrealistically aggressive gaps, especially when decelerating. Next, some experimental results, using the above algorithms, will be analyzed.

\subsection{Experimental Results}

The initial simulation results obtained, using the above algorithms, provided some insight into the behaviour of SVs. Each experiment involved 100 simulations, each simulation consisted of 1800 iterations, and were run with road loops of 1000, with cars able to take a maximum speed of 5 cells per iteration.

A method of interpreting these constants is, assuming a road has a maximum speed of $100 \mathrm{~km} / \mathrm{h}$, each additional speed from 1 to 5 represents an increase of $20 \mathrm{~km} / \mathrm{h}$, and 1800 iterations represents 30 minutes of driving time. Also assuming that each iteration represents the decision made in one second on the roadway, each cell then represents roughly 5.56 meters and therefore the entire road loop is a little longer than $5.5 \mathrm{~km}$. This also means that if a car is travelling at the maximum speed for the whole experiment, it will traverse the entire road loop just over 9 times. Of course 
each iteration can also represent two seconds of real-time, which would adjust all the interpretations above. In such ways, these results can be translated to different situations if required for an application.

For every experiment, the graphs displayed averaged the results (based on the Criteria given in Section 3.4) of all simulation in each experiment for each iteration, and belong to one of the following three categories:

1. Speed-Graph (SG): This graph displays the average speed of all cars on the road, or average road speed, and the optimal average speed of the entire road in the experiment, per iteration. The optimal speed is calculated as the minimum of the maximum speed of the road, and the total number of cells on the road divided by the number of cars at the start of the iteration. Clearly, for closed roads, this optimal speed remains constant, and for open roads, this can change depending on the number of cars that enter or exit on any iteration.

2. Change-Graph (CG): This graph displays the absolute sum of speed changes divided by the number of cars, per iteration, and in multi-lane cases, it separately plots the number of total lane changes divided by the number of cars, per iteration. In the multi-lane case, the speed changes are shown on the left, and lane changes are shown on the right.

3. Dynamics-Graph (DG): This graph displays the percentage of jammed cars, and also the percentages of cars with a large Front Gap. There are three lines for jammed cars, labelled as jammed, representing the percentage of cars that are travelling slower than the optimal speed, group jam, representing the percentage of cars belonging to a group of 5 consecutive cars with a front gap less than the optimal road speed, and stopped, which is the percentage of cars travelling at speed 0. There are also two lines for optimal cars, labelled as optimal, representing the percentage of cars travelling with speed exactly equal to the optimal road speed, and large gap, which is the percentage of cars with Front Gap larger than or equal to the maximum speed of the road. For multi-lane cases, there is also a faint yellow line representing the percentage of cars in the first lane. 
The experiments are also differentiated by the density of the road, which is de-

fined as the number of cars divided by the number of available road cells: $\frac{n C}{\left(R_{\text {len }}\right)\left(R_{\text {lanes }}\right)}$. Because the road length, $R_{\text {len }}$, remained constant for all experiments, it was possible to control the density of the road by changing the target number of cars on the road. A sparse road refers to a road with density less than or equal to $\frac{1}{\text { maxSpeed }}$, suggesting, in theory, that all cars could maintain a Front Gap larger than the maximum speed, unless one of the cars was forcibly slowed down. Alternatively, a dense road is defined by having a density greater than or equal to $\frac{1}{\text { maxSpeed }}$, suggesting that all cars absolutely could not be travelling at the maximum speed on any iteration, without the need for them to slow down during that same iteration to remain collision free. In the experiments below, sparse roads are defined as roads with a 0.1 density, and dense roads are defined as roads with a 0.2 density.

The experiments consisted of different types of cars: one for each of the three car types explained in Section 3.2.3, and one with a uniformly-sampled combination of the three types, referred to as the "Mixed" type. All graphs specify in the title, legend, or caption, which type of graph (SG, CG, or DG), how many lanes the road has, and also how many and what type of cars are on the road. The sections below cover the analysis of the single lane (SL) and double lane (DL) experiments with fixed target densities to determine the baseline traffic statistics.

\subsubsection{Single-Lane Results}

The first experiment in this section compared sparse and dense closed single lane roads, and the second experiment evaluated the impact of perturbing cars also in both sparse and dense closed single lane roads.

\section{Sparse and Dense Closed Single Lane Roads}

As seen in the left of Figure 4.2, which shows the Sparse Road Speed Graph, the experiment with exclusively aggressive cars reached the maximum speed first, followed by the experiment with normal cars, then mixed cars, and finally the experiment with passive cars. The passive cars also converged to the maximum speed after around 750 

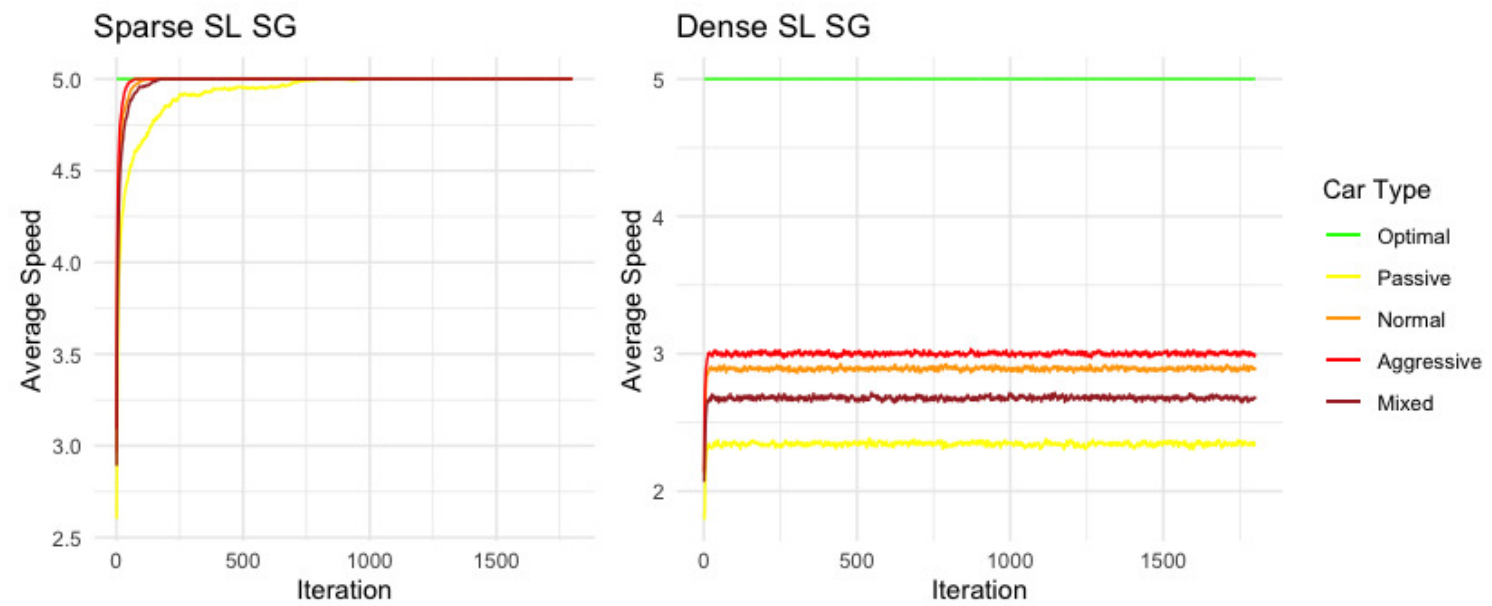

Figure 4.2: The Speed Graph (SG) comparing the experiments for sparse single lane roads with a 0.1 density (left), and dense single lane roads with a 0.2 density (right), both for all four car types.
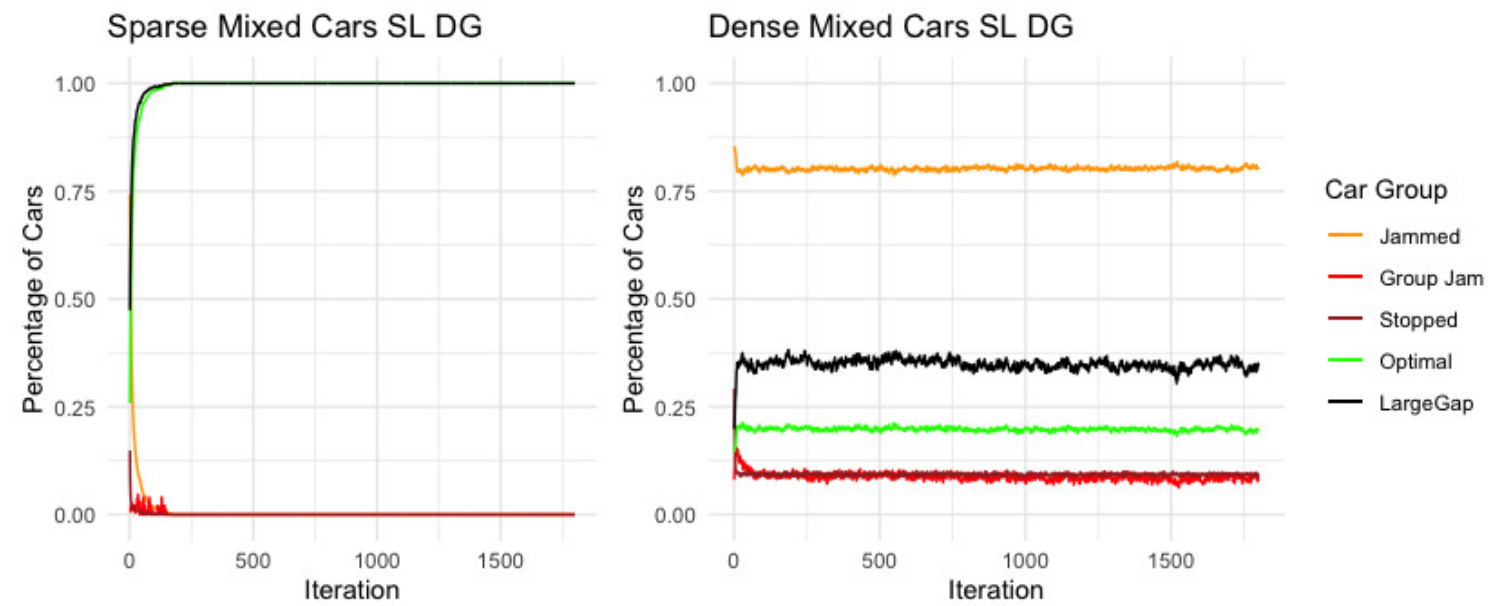

Figure 4.3: The Dynamics-Graph (DG) for the sparse (left) and dense (right) single lane road experiment consisting of mixed car types, represented by the dark red line in Figure 4.2. 
iterations. The discrepancy between car types was further evident in the Dense Road experiments on the right of Figure 4.2, where experiments containing aggressive and normal cars converged to a speed under 3, while the experiments containing mixed and passive cars settled at an average speed around 2.7 and 2.3 units per iteration.

This shows that in single lane roads (where cars only have to decide their speeds), all car types fairly quickly reached the maximum speed if the road is sparse. In the Dynamics-Graph for sparse roads, shown on the left in Figure 4.3, some initial group jams occurred, but appeared to quickly disperse, as seen by the spiky behaviour of the red line in the graph.

Conversely, in the dense road experiments, while the average speed of the cars converged for all four experiments, there were still a consistently large proportion of both stopped cars and optimal cars, as seen in the Dynamics graph on the right of Figure 4.3. This occurred because individual cars continued to change their speeds quite often, going through the stop-and-go waves caused by emergent traffic jams. For this road to operate optimally, at most very few cars should be travelling at the optimal speed; in fact, all cars travelling at speed 4 and spaced with Front Gaps of at least 5 would result in the need for no car to accelerate or slow down. Because cars are only made aware of their local information, these stop-and-go waves are hard to prevent using any stochastic rule-set where cars prioritize maximizing their own speeds, and not the average speed of all cars on the road.

\section{Sparse and Dense Closed Single Lane Roads with Perturbations}

The second experiment evaluated the impact of randomly perturbing the road. To accomplish this, on every 30 iterations, every 100th road cell was given a maximum speed of 0 for a single iteration, forcing any car on that road cell at the start of that iteration to fully stop. As seen in the speed graph in Figure 4.4, there were noticeable downward spikes at seemingly fixed numbers of iterations. Randomly stopping a car for one iteration did not affect the overall average road speed unless it forced cars that were behind it to slow down or to completely stop as well, creating a cascade effect. In sparse road situations, once cars reached their maximum speeds, the perturbations no longer noticeably affected the average speed of the road. In the 

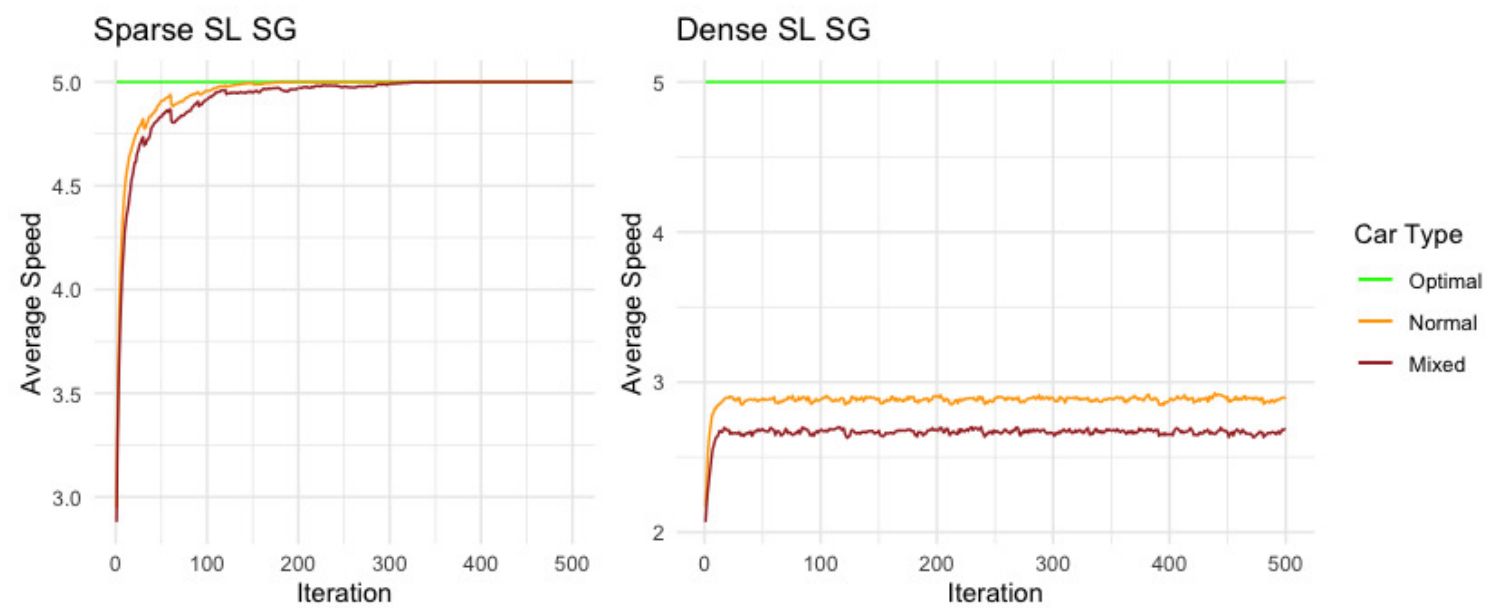

Figure 4.4: The Speed Graph (SG) comparing experiments for sparse single lane roads with a 0.1 density (left), and dense single lane roads with a 0.2 density (right), where all experiments involved perturbing the road every 30 iterations on every 100 cells.

dense road, perturbations still did not affect the overall average speed of the road, likely because there were already a high proportion of stopped cars due to the stopand-go waves. After the 500 iterations displayed the behaviour stayed consistent, but the reason for only plotting 500 iterations was so that one could observe slight decreases in speed every roughly 30 iterations.

The single lane experiments in this sub-section demonstrated how, in sparse closed roads, SVs fairly quickly reached optimal speeds after starting with randomly chosen speeds and gaps. This suggests that the algorithms used to design SVs are sufficiently aggressive. In dense roads, the cars appeared to converge to an average speed that was more than one unit lower than the theoretical maximum, and therefore has room for improvement. Finally, randomly perturbing cars did not seem to impact the convergence speeds of cars on single lane roads.

\subsubsection{Double-Lane Results}

The first experiment in this section compared sparse and dense closed double lane roads, and the second experiment compared open and closed double lane roads. 

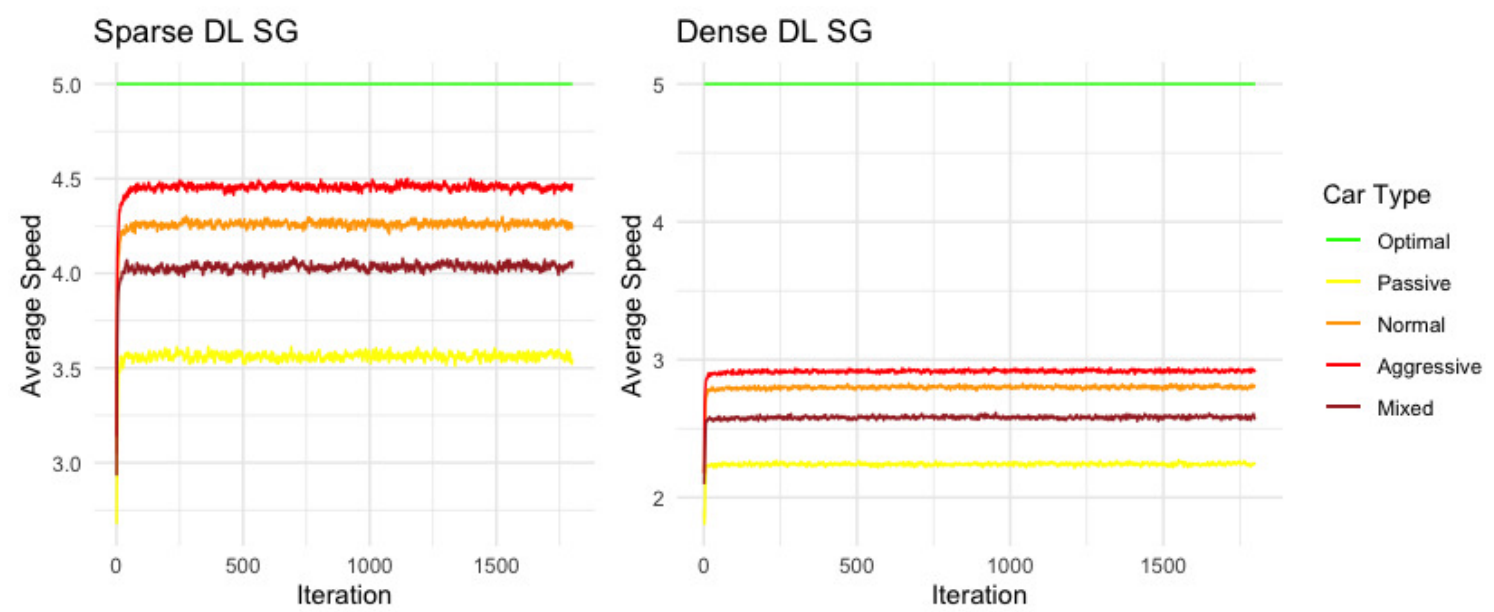

Figure 4.5: The Speed Graph (SG) comparing sparse double lane roads consisting of a 0.1 density (left), and dense double lane roads consisting of 0.2 (right). Note that twice as many cars are needed in a double lane road to reach the same density as a single lane road, because density is proportionate to the total number of road cells and not the road length.

\section{Sparse and Dense Closed Double Lane Roads}

The closed double lane roads had the same densities as the sparse and dense single lane experiments. Surprisingly, the sparse road experiments did not reach maximum speeds for any type of car, as seen in the speed graphs shown in Figure 4.5. Adversely, to the change in sparse roads, cars were only slightly slower in the dense double lane road experiments, compared to the dense single lane road experiments. This is not as expected, because, in theory, the cars had more than enough of a Front Gap to accelerate more, especially in the sparse road case. This demonstrates how difficult it is for cars that are behaving greedily to change lanes efficiently.

\section{Comparing Different Densities in Open and Closed Roads}

The second set of experiments compared open and closed double lane roads at four density targets, labelled by the different target numbers of cars in each experiment, in the Speed Graph shown in 4.6. At the target density 0.05, in the closed road, all cars very quickly reached the optimal speed of the road. For open roads, reaching a 

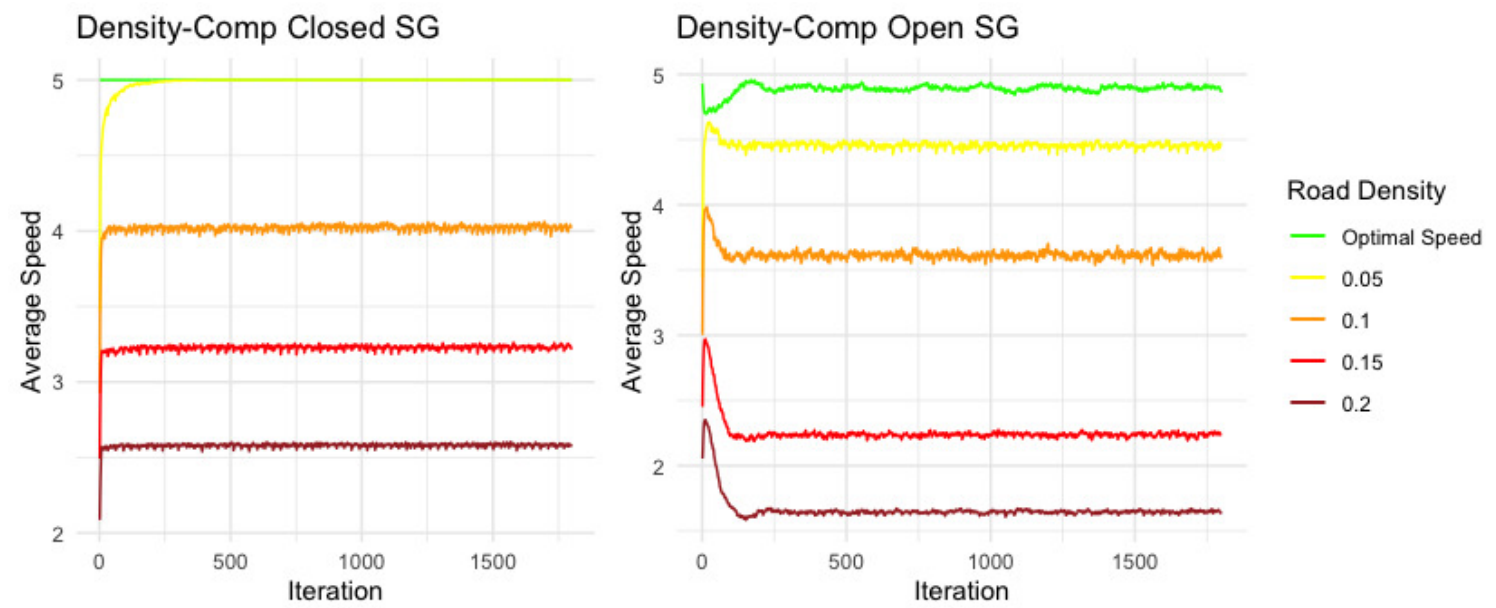

Figure 4.6: The speed graph (SG) for closed roads (left), and open roads (right) for four experiments with $0.05,0.1,0.15$, and 0.2 densities, all of mixed types. The open roads consisted of 5 exits and entries, with an exit-rate of 0.005 , meaning each car had an expected exit time of 200 iterations. Furthermore, for both open and closed roads, cars were randomly perturbed, similar to the single lane scenarios.

maximum speed, on average, on any iteration, was unlikely, since cars enter the road with a randomly selected speed. However, cars in the open road did converge to an average speed, which was noticeably less than the closed roads with the same density.

The Change-Graphs for speed and lanes for closed roads at different densities, displayed in Figure 4.7, show that roads with lower densities had less speed changes, and proportionally more lane changes. This is likely because cars in sparser roads chose to change to a faster lane and maintain their speeds, rather than decelerating and waiting for their Front Gaps to increase. For example, it can be seen that, at density 0.05 , even though cars reached the maximum speed on average, around $25 \%$ of the cars continue to make a lane change on every iteration on average.

Comparatively, the change graph for open roads, shown in Figure 4.8, suggests that the lane-changing pattern was very similar to the closed-road graph, but the speed-changing averages were quite different. In the open-road case, the average number of speed changes for the 0.2 and 0.15 density experiments were much lower than in the closed road experiments.

The underlying behaviour is easier to observe when comparing the dynamics graph 

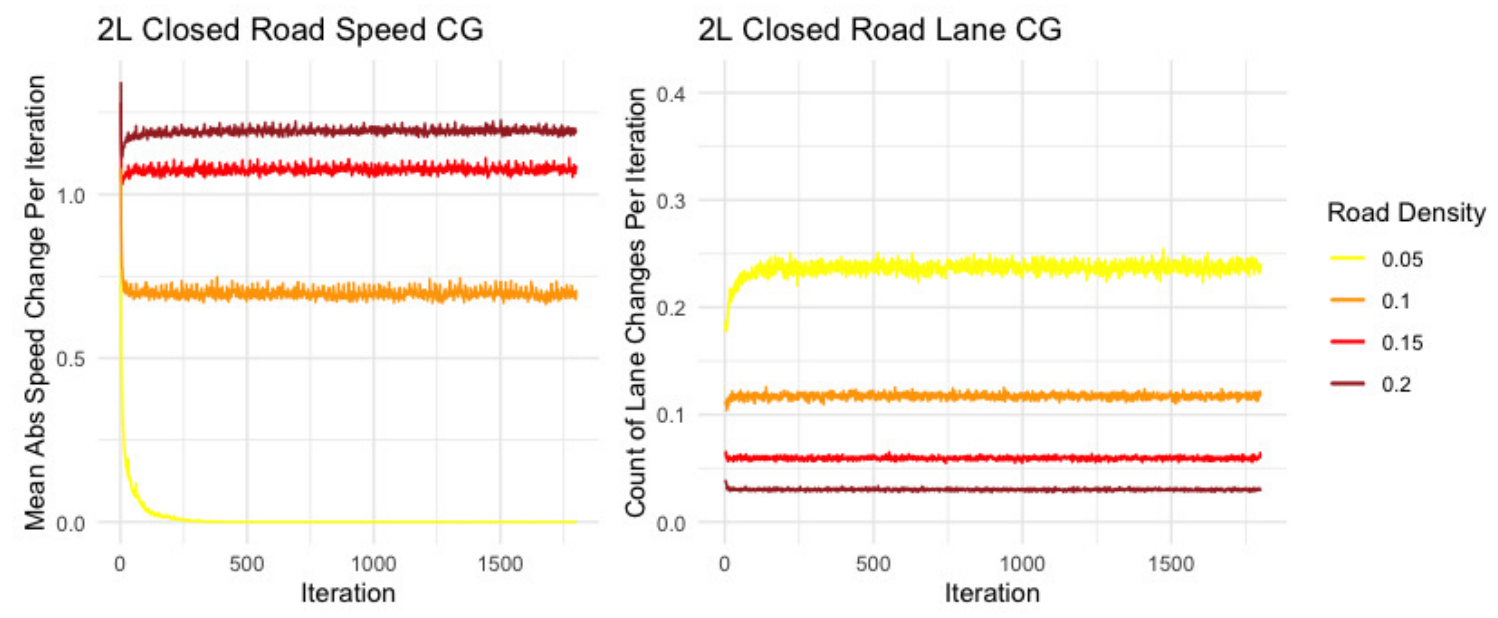

Figure 4.7: The change-graph for all four closed road double lane experiments, with speed on the left and the number of lane changes on the right.

2L Open Road Speed SG

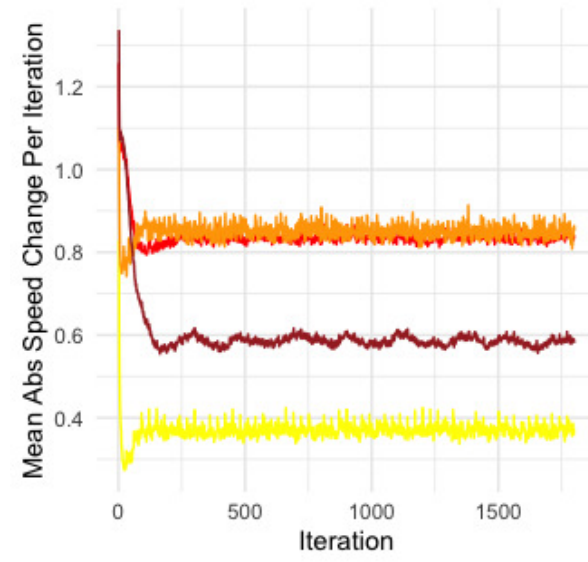

2L Open Road Lane SG

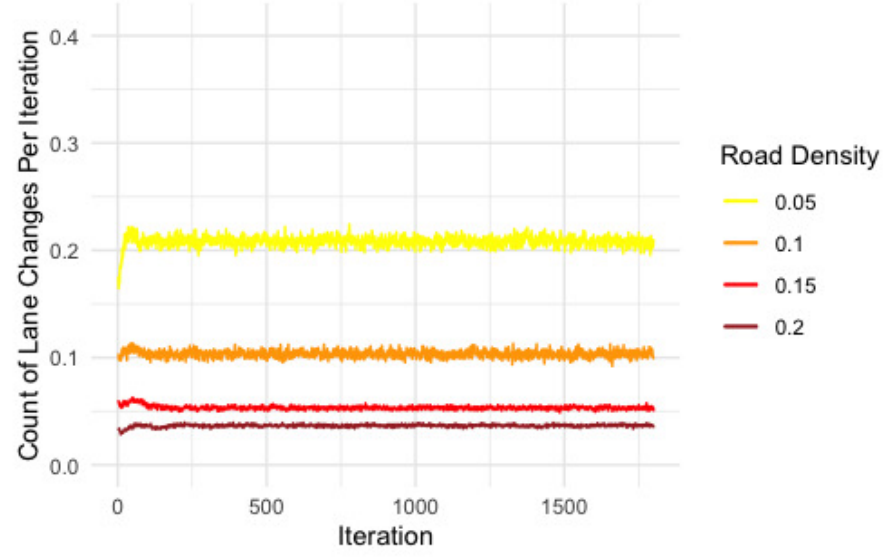

Figure 4.8: The change-graph for all four open road double lane experiments, with speed on the left and the number of lane changes on the right. 

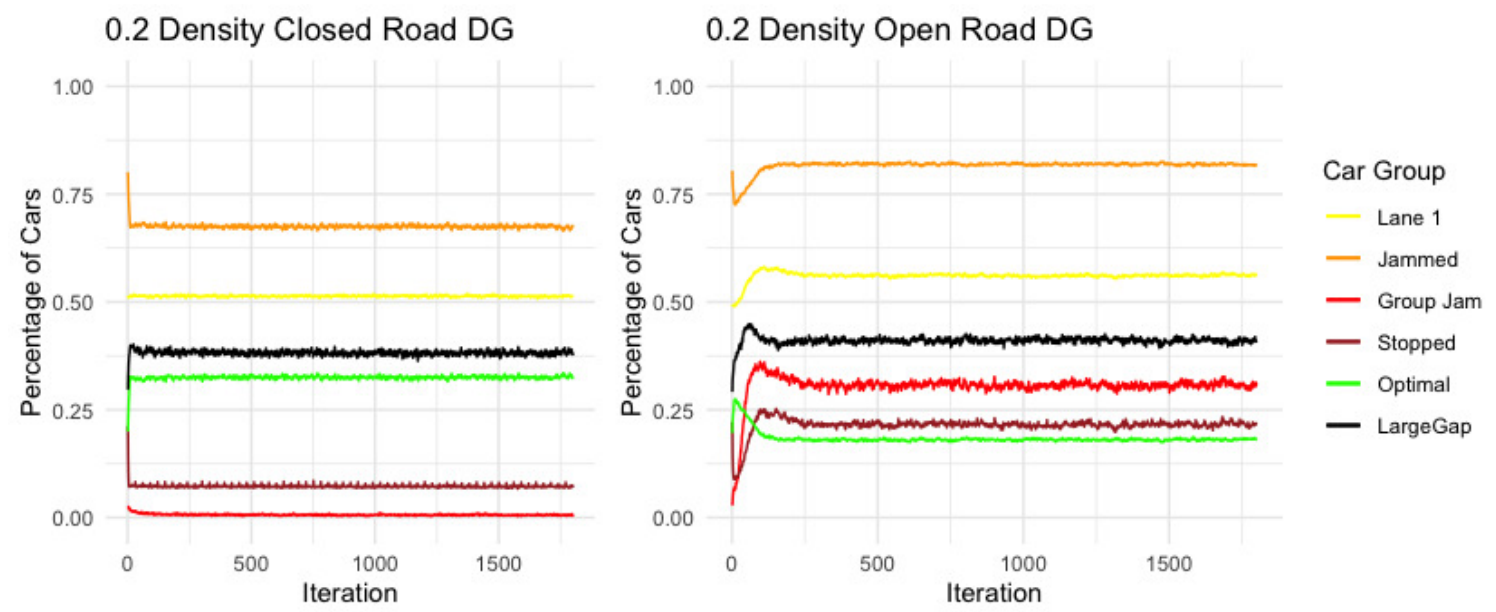

Figure 4.9: Left: The dynamics graph for the 0.2 density closed road double lane experiment (left) and open road double lane experiment (right).

for open and closed roads for both 0.2 density experiments, shown in Figure 4.9. There was a higher percentage of stopped cars and cars in group jams in the open road case compared to the closed road case. This suggests that many cars stayed jammed for a longer duration, which reduced their ability to accelerate enough to raise the average number speed changes on the road overall. This appears to be because cars were unable to safely change out of the first lane as frequently as in the closed road, therefore creating a higher percentage of total cars in the first lane, and as a result, creating more group jams.

In summary, both open and closed road double lane results with a density equal to or greater than 0.1, along with the dense single lane results, converged to average speeds that were lower than their theoretical maximum possible speeds, and therefore have room for improvement.

\subsubsection{Extensions to More Road Settings}

Experiments with $n$-Lane roads are given in Appendix B.1, to further examine road dynamics as the number of lanes continued increasing. Furthermore, some experiments with density targets changing during the experiment, to simulate "rush hour" traffic, are given in Appendix B.2. While these $n$-Lane experiments also have room 
for improvement, the general concepts presented in Chapter 5 for training AVs can be feasibly translated to higher lanes, and therefore $n$-Lane roads will not be covered with $\mathrm{AVs}$ in this thesis.

The main differences in $n$-Lane roads compared to single and double lane roads occur especially in open road settings. Specifically, the percentage of cars in the first lane increases much more drastically compared to what it should be, both in sparse and dense roads. This, once again, creates a larger density in the first lane, and so, cars do not switch out of the first lane as efficiently as they could.

\subsection{Main Results}

The following are a summary of all the main results from the stochastic experiments run in this chapter:

1. Closed Sparse Single Lane Roads: All cars converged to the maximum speed.

2. Closed Dense Single Lane Roads: The baseline average speed for SVs with mixed car types was 2.68, the percentage of cars stopped was $0.094 \%$, and the mean absolute number of speed changes was 1.03 .

3. Closed Sparse Double Lane Roads: The baseline average speed for SVs with mixed car types was 4.02 , the percentage of cars stopped was $0.042 \%$, and the mean absolute number of speed changes was 0.70 .

4. Closed Dense Double Lane Roads: The baseline average speed for SVs with mixed car types was 2.58, the percentage of cars stopped was $0.1 \%$, and the mean absolute number of speed changes was 1.19.

5. Open Sparse Double Lane Roads: The baseline average speed for SVs with mixed car types was 3.33, the percentage of cars stopped was $0.075 \%$, and the mean absolute number of speed changes was 0.88 . Calculating the theoretical maximum of an open road is non-trivial, but an approximation can be obtained as follows: in a road with density 0.1 , and a 100 total of cells for entering and 
exiting, with cars entering the road at an average speed of 2.5 units, this suggests the theoretical maximum speed is roughly 4.875 . Note that it is unlikely that the road will ever attain to this theoretical upper bound.

6. Open Dense Double Lane Roads: The baseline average speed for SVs with mixed car types was 1.65 , the percentage of cars stopped was $0.45 \%$, and the mean absolute number of speed changes was 0.59. Similar to the open sparse roads, the theoretical maximum speed of a closed dense road is approximately 3.85 , though this will also be close to impossible to reach.

\subsection{Conclusions}

This chapter began by providing the algorithms used to model SVs, which were designed to mimic the behaviour of human drivers. The models used were a variant of NS traffic modelling, but provided cars with a wider range of actions to take, and also differentiated the cars into 3 distinct types, to account for diverse driving behaviours. These results demonstrate a behaviour as expected, when compared to the literature covered in Section 2.2. Because of the additional aggressive behaviour permitted, these vehicles are able to move from jammed to jam-free states very rapidly.

Experimental results on different road conditions were also displayed in this chapter, to get an empirical idea of the road dynamics created by SVs. In Appendix A.2, some of the convergence values of these experiments are given in tabular form. The main conclusions are that cars behaving greedily did not change lanes efficiently, therefore causing many jams for the vehicles behind, and resulting in significantly slower overall average speeds than what were theoretically possible. Furthermore, it can be seen in open double lane roads, where entering cars ended up creating many slowdowns in the first lane of the road (especially in dense roads), that this inability to efficiently change lanes created many emergent jams, especially in the first lane. Road conditions actually improved slightly in 3 and 4 lane roads, compared to the change from single lane to double lane roads, and these results can be seen in Appendix B.1.

It does appear that in terms of the primary and secondary criteria given in Section 
3.4, SVs tend to converge. Thus, it is possible to determine how AVs impact the road, by replacing some SVs with AVs, and directly comparing these criteria. This is evaluated in the next chapter, using the same underlying road conditions from the experiments above. 


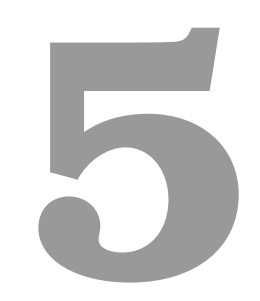

\section{Mixed-Autonomy Traffic Patterns}

\section{$5.1 \quad$ Introduction}

Mixed-autonomy traffic environments occur when both autonomous vehicles (AVs) and stochastic vehicles (SVs) travel on the same road. In this thesis, such traffic is modelled assuming that AVs do not enter or exit the road, but instead just loop repeatedly for the duration of the experiment. AVs, like SVs, are made aware of their gaps with adjacent cars, and are also forced to make decisions that do not result in collisions. AVs, unlike SVs, make decisions by feeding past experiences into an RL algorithm designed to maximize the overall road speed, rather than follow a set of rules. As was the case in the the previous chapter, the focus in this chapter will be on analyzing single lane closed road experiments, and thereafter, double lane open and closed road experiments. In all experiments, the SVs are of mixed types, and the mixed-cars SV experiments from Section 4.3 are used as the benchmarks. 
This chapter begins by providing general explanations of how the AVs were trained using RL, specifying the reward functions used, and other factors that are universally applied to all experiments, in Section 5.2. After that, the results of Single Lane Experiments are presented in Section 5.3. The results of AVs that do not change lanes in Double Lane Experiments are reported in Section 5.4, and finally the results of AVs that do change lanes in Double Lane Experiments are analyzed in Section 5.5. The extensive results in table form are provided in Appendix A.

\subsection{Defining the RL Environment}

To re-define the traffic navigation problem as an RL problem, it is important to define the actions that each AV can take, the state space associated with each type of experiment, the reward functions used, and the initial placement of the AVs. The actions, similar to those described in Section 4.2, are restricted to be collision-free, and therefore all of the constraints which apply to SVs also apply to AVs. The difference between the SVs and AVs is the selection process of the action from the set of available actions during each iteration. The state space is dependent on the underlying road environment and the type of $\mathrm{AV}$, and this will be further emphasized in the respective sections below. For all the autonomous vehicles modelled in these sections, the following five reward functions are tested:

1. RF1: The speed of the AV.

2. RF2: The sum of RF1 for all AVs on the road.

3. RF3: The minimum of the Front Gap, Back Gap, and Speed of the AV.

4. RF4: The sum of RF3 for all AVs on the road.

5. RF5: The current speed of the AV subtracted by the absolute change in speed of the car between the current and previous iteration.

Note that RF1, RF3 and RF5 suggest that each vehicle aims to maximize a cumulative reward which depends on its own behaviour, while RF2 and RF4 depend 
on the behaviours of all AVs on the road. This provides a comparison between AVs that are focused on maximizing their own local situation, to AVs that are making decisions to maximize an approximation of the speeds of the entire road, and by extension, minimize the adverse downstream effects on the other AVs.

Finally, it is also important to consider the placement of the AVs, specifically differentiating between AVs placed consecutively in a line with no SVs in between, referred to as Type 1, and AVs randomly placed on the road, separated by SVs, referred to as Type 0. In Sections 2.2 and 2.4, there was analysis of previous papers that demonstrated how placing AVs in a Type 1 orientation had the potential to lower the impact and duration of emergent traffic jams. However, these experiments were run with different state and action spaces, and in the methodology presented in this chapter, it would seem intuitive that a Type 0 pattern should perform better. Furthermore, in double lane roads, it is feasible and very common for a road to begin in a Type 1 orientation, and finish in a Type 0 orientation, because the vehicles were broken up due to an SV changing lanes or passing one of the AVs.

\subsection{Single Lane Experiments}

In single lane experiments, cars do not have to make lane decisions, and therefore choose from a small, finite set of actions, which are only constrained by the Front Gap of the road cell which the car is on. As seen in the section on SVs, dense road experiments are where cars begin to make inefficient decisions, and therefore, the results displayed below are for experiments run in dense road settings. First, experiments using tabular RL are reported, followed by approximate RL methods that use regression and decision-tree based function approximation. Finally, the results using Deep Q Networks are are presented.

\subsubsection{Tabular Reinforcement Learning}

Tabular RL is executed using the Q Learning algorithm described in Section 2.3.4. The target $\mathrm{Q}$ value is calculated as the maximum predicted $\mathrm{Q}$ value of all actions from 


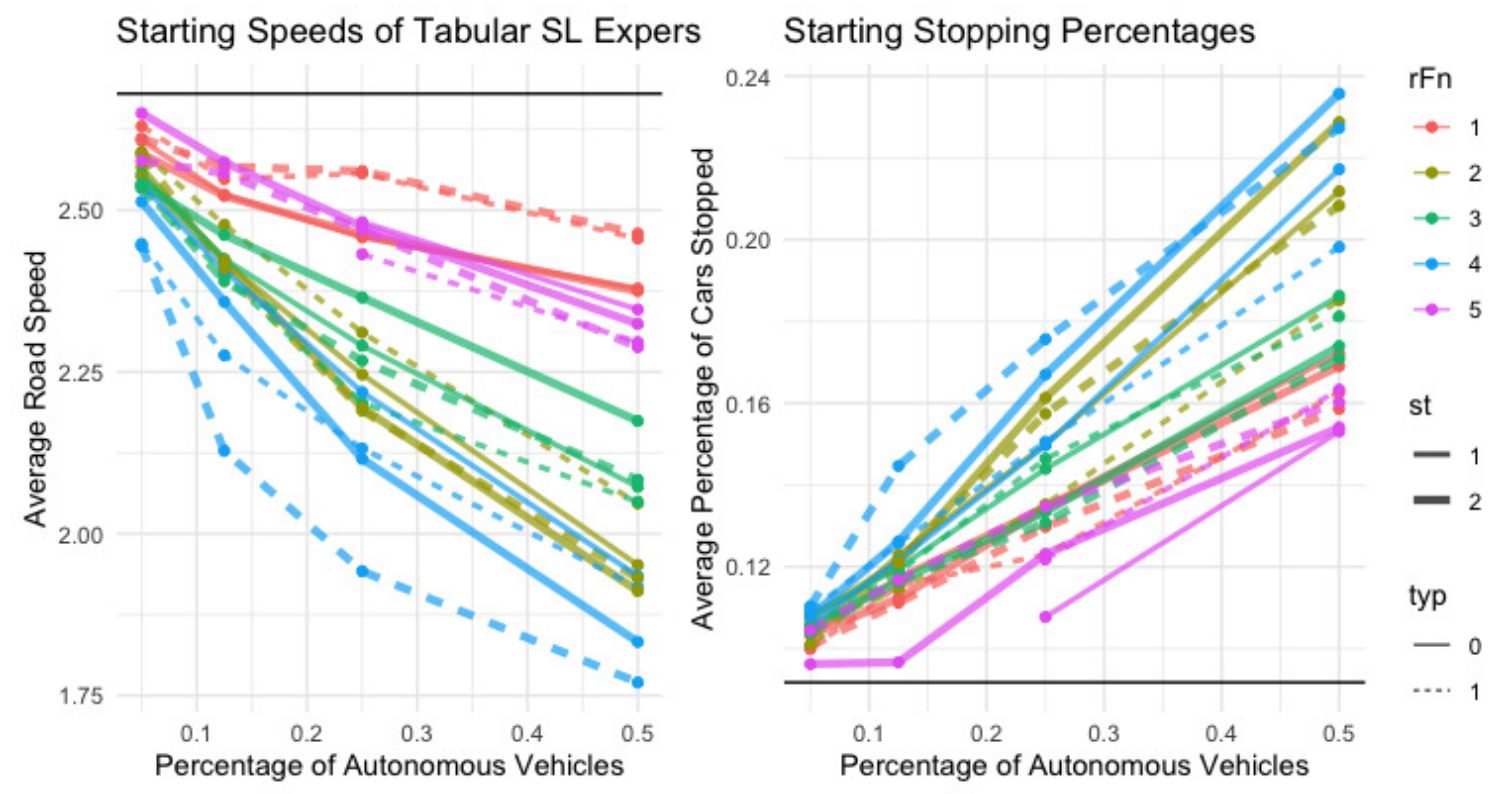

Figure 5.1: The Average Speed of all cars on the road (left) and percentage of cars that are stopped (right) during the exploration phase of tabular single lane RL experiments, including the SV convergence value (horizontal black line).

the new state, added to the reward received at the previous time step. Two different state spaces were used: the first was the minimum of the Front Gap and Maximum Speed of the road cell that the AV was on, and the second was the minimum of the speed picked by the AV, the Front Gap, and the Back Gap of the road cell that the AV was on. Therefore, both state spaces that were used had a total of 6 states, which allowed each car to adequately explore all state-action pairs. Larger state spaces were tested, including combinations of previous speeds, Front Gaps, and Back Gaps, but these did not improve the results. Each vehicle updated a common state-action table, meaning the AVs were indirectly learning from each others' experiences.

All the tabular experiments were initialized with $\alpha=0.5, \epsilon=0.5, \lambda=0.2$, and $\gamma=0.5$, and run for 500 iterations with these initial settings to allow for many iterations of exploration. Afterwards, they were run for 1300 more iterations, with a decay rate for both learning and exploration of 0.999 per iteration. Typically, the table values for each experiment converged after 300 to 500 iterations after the exploration phase was finished. By the end of each experiment, the exploration rate 


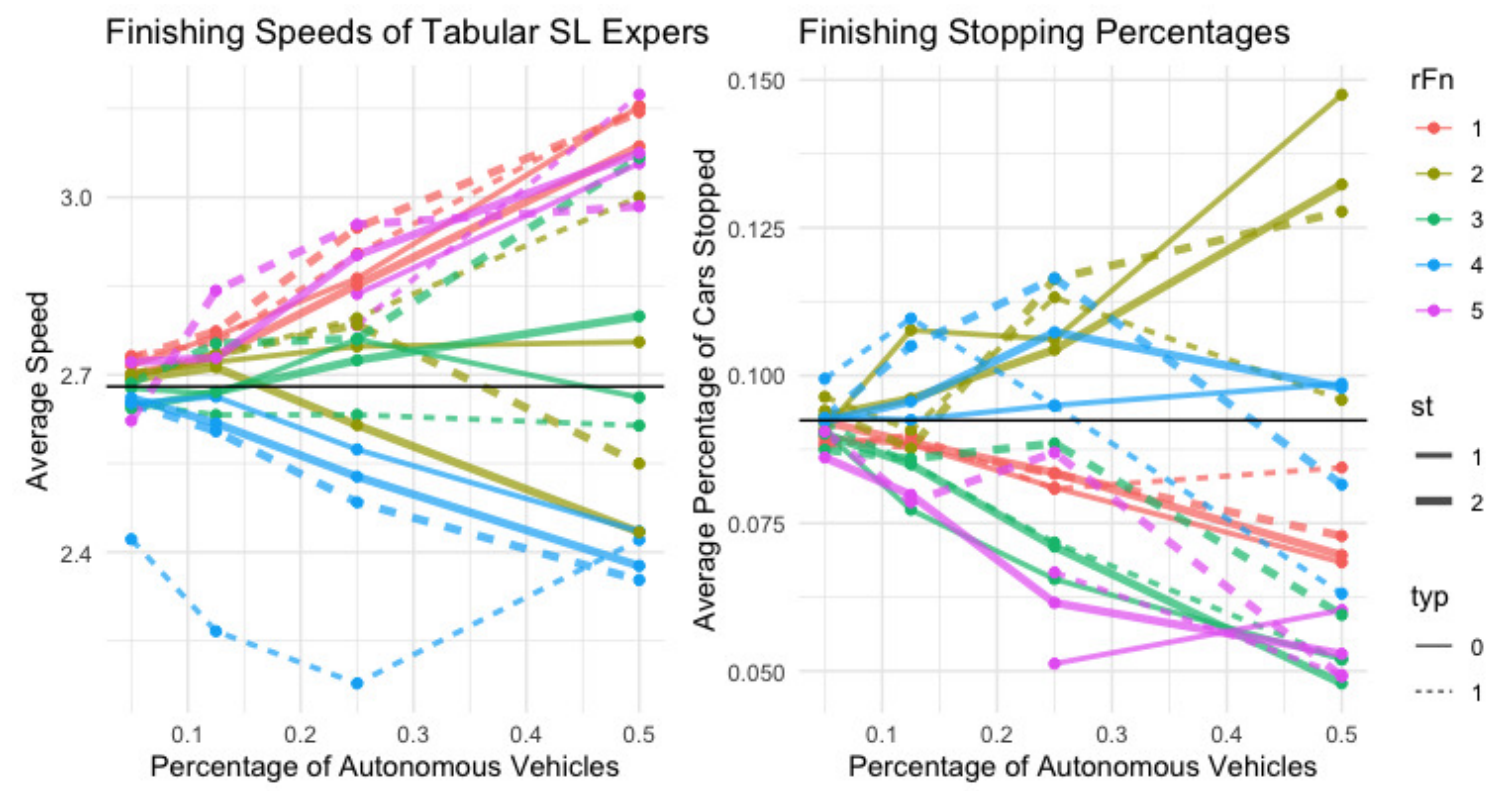

Figure 5.2: The Average Speed of all cars on the road (left) and percentage of cars that are stopped (right) once the table values converged for tabular single lane RL experiments, including the SV convergence value (horizontal black line).

was low enough to remove the majority of the variability in action selection, so that the overall road speed averages converged for the overall road as well. For tabular roads, the experiments were individually done for each of the 5 reward functions, both state spaces, both types of initial AV placements, and initial percentages of AVs equalling $0.05,0.125,0.25$, and 0.5 .

The graphs in Figure 5.1 display the average speed of all cars on the road, and the percentage of all the cars on the road that are travelling with speed 0 , averaged during the exploration phase. The dense road experiments, with only SVs, converged to an average speed of 2.68 cells per iteration, as determined in Section 4.3, which will be referred to as the benchmark for single lane roads, and is represented by the black horizontal line in each of the graphs. Based on the stopping percentages shown on the left, it can be seen that many of the cars initially slowed down well below the benchmark. It does appear, based on the graph representing stopping percentages, that, at first, cars following RF5 chose to stop the least, likely to avoid the reduced reward from quickly-changing speeds. 
The graphs in Figure 5.2 display the speed and stopping percentages at the end of the experiment, after the exploration rate had decayed below $1 \%$, to avoid the noise from arbitrary slowdowns. As is clearly visible, RF1 and RF5 perform the best in tabular settings, in terms of maximizing average road speed, while RF2 and RF4 perform significantly worse. RF3 is interesting, because it does not, for the most part, perform better in terms of average speed, but does consistently reduce the percentage of cars stopped. This occurred because the resulting convergence tables for RF3, and also RF5, placed a higher table value for choosing the speeds 3 and 4 even when the Front Gap was large, while RF1 performed well because it constantly chose the largest available speed, and yet still caused downstream disturbances, leading to proportionally more stopped cars. Finally, the second state definition performed better in general, though the first state definition had the best overall individual result with RF5, and Type 0 appears to perform slightly better, in general, for most experiments.

As is clearly visible, the best results show that once $50 \%$ of the road is converted to AVs, the entire road increased over $40 \%$ closer to the theoretical maximum possible speed that could occur. Furthermore, adjusting just $5 \%$ of the vehicles to be AVs could potentially improve the entire road to being $4 \%$ closer to the theoretical maximum speed, though this only applied to the reward functions that performed well. Given the nature of the SVs, the maximum theoretical speed was really only achievable if every single vehicle was perfectly evenly spaced. This was extremely unlikely to occur, and therefore the improvement demonstrated is quite substantial!

Note that in tabular single lane roads, fully populated by AVs, the average speed of the dense road converged to exactly 4 units using RF1 or RF2, equal to the theoretical optimal. However, this was not achieved by perfect spacing; instead, the cars favoured the maximum speed or a speed equal to 4 if the Front gap was large enough, and otherwise preferred to fully stop for one iteration. This eventually led to roads where all of the cars travelled at their maximum speeds except the few that were stopped on each iteration, such that the average overall speed was the theoretical maximum speed of the road. 


\subsubsection{Approximate Reinforcement Learning Methods}

Two approximate RL methods were initially tested including regression-based, and decision-tree based. In the single-lane approximate road cases, the input features used to train the underlying function approximation algorithm were:

1. The three previous usable gaps of the vehicle, calculated as the minimum of the Front Gap, and the maximum speed of the road cell the AV was on.

2. The three previous actual Front Gaps.

3. The three previous actual Back Gaps.

4. The three previous speeds taken by the car.

5. The speed chosen by the car on the current iteration, which is also the action taken by the car.

6. The type (autonomous or stochastic) of the vehicle ahead immediately ahead (in the same lane).

This created a feature set of size 14, designed to provide the snapshots of the vehicle at the previous three time-steps, allowing, if required, for the function approximator to implicitly calculate its own acceleration. Furthermore, the full feature-set after each iteration was saved into the replay buffer, allowing other cars to learn from experiences in different parts of the road and from different cars. Note that in this set of experiments, the place of each car and the iteration when each car reached that place on the road were not saved to the replay buffer, so as to prevent the AV from learning and taking advantage of the specifics of the road construction.

For both the regression and decision-tree experiments, the sample size from the replay buffer on each iteration was 200 for experience replay, and then increased gradually up to 3000 by the end of the experiment. Furthermore, the fixed target weights were updated every 20 iterations at the beginning, and this also increased gradually up to 50 iterations by the end of the experiment. The experiment was initialized with $\alpha=0.5, \epsilon=0.5, \lambda=0.5, \gamma=0.1$, with $\alpha$ and $\epsilon$ decaying by 0.999 , and the gamma 

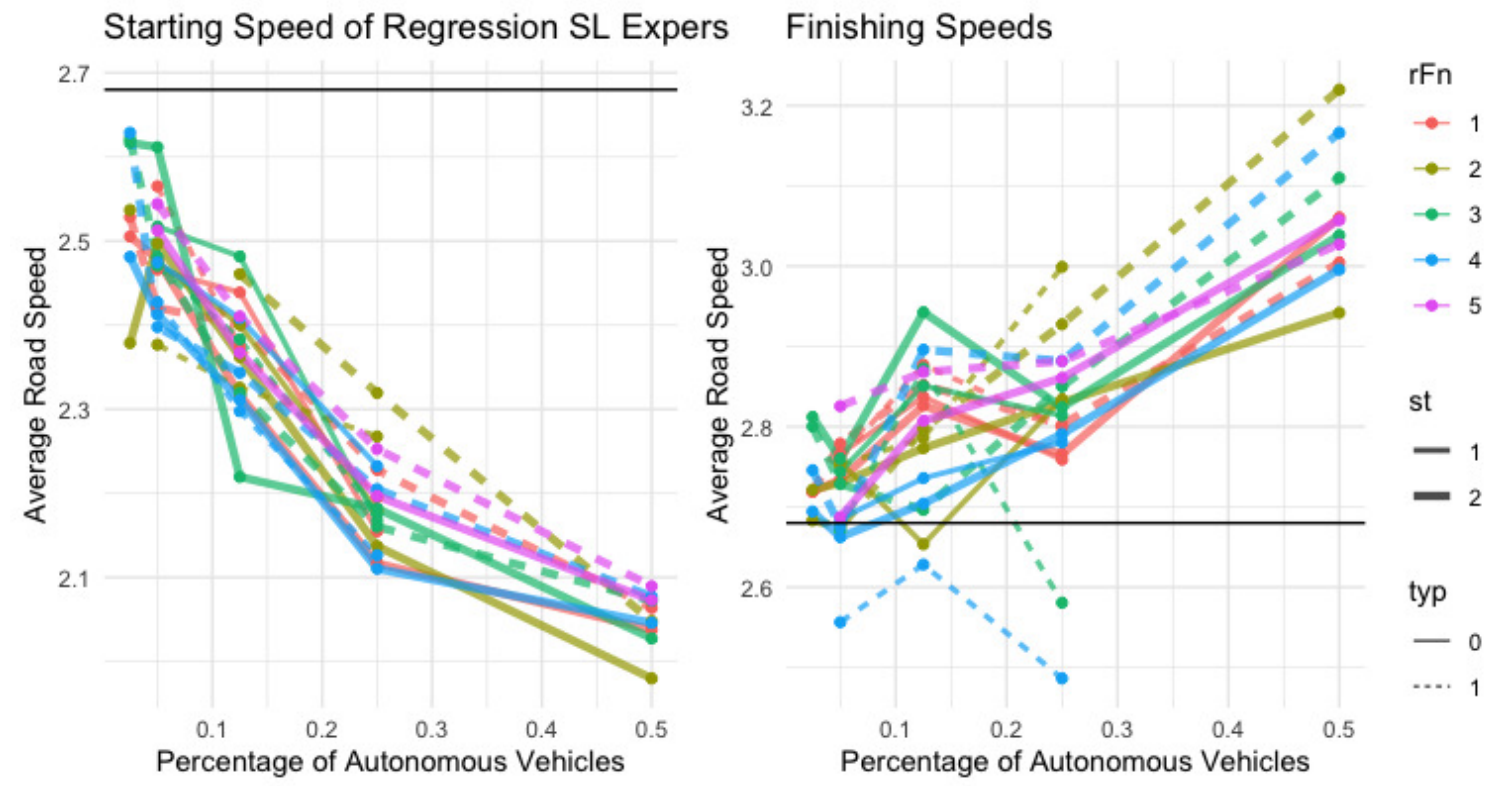

Figure 5.3: The exploration phase (left) and convergence (right) Average Speeds for Linear-Regression Based Single Lane RL experiments, including the SV convergence value (horizontal black line).

value increasing by 1.001 on each iteration. The first experiment was run using linear regression as the approximation algorithm, using eligibility traces where the lambda value was held constant throughout the experiment. The second experiment used XGBoost, which is a decision-tree based algorithm, and because the weights for each feature were no longer linearly combined with the feature values, eligibility traces could not be applied. Furthermore, to build the initial replay buffer, vehicles began by randomly selecting from the set of available actions for 100 iterations. Note that these iterations are not recorded in any of the graphs given below.

\section{Linear Regression Based Function Approximation}

As can be seen in Figure 5.3, it appears that with lower percentages of AVs, RF3 and RF5 seem to have a higher average speed. With higher percentages of AVs, it appears that RF2, and RF4 resulted in a higher average road speed. Furthermore, it appears that in the regression experiments, placing AVs in Type 1 orientations, where the AVs are adjacent to each other, yielded a generally higher average road speed, and 


\section{Starting Speeds of XGBoost SL Expers Finishing Speeds}
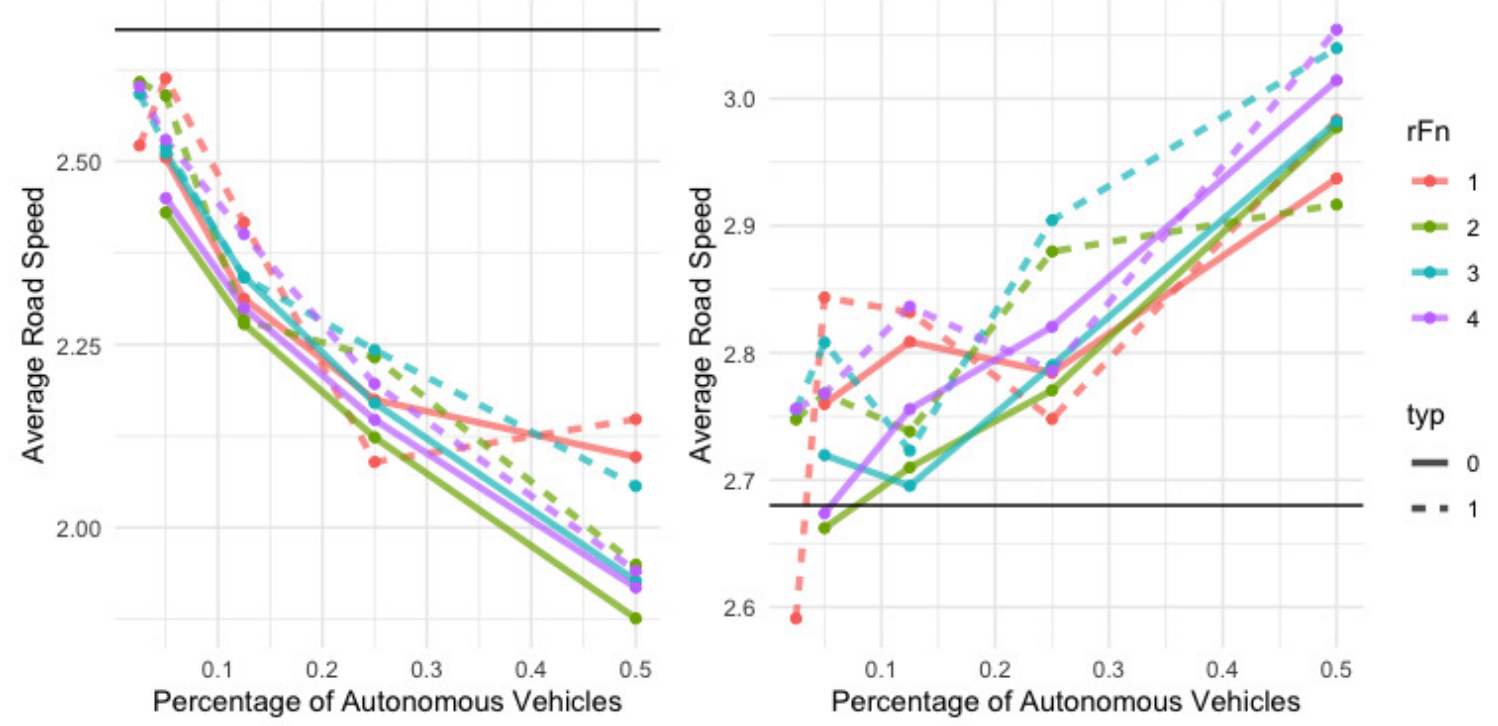

Figure 5.4: The exploration phase (left) and convergence (right) Average Speeds for XGBooost Based Single Lane RL experiments, excluding RF5, because the results were poor (included average speeds under 2.5).

that unlike the tabular experiments, all reward functions performed better than the experiment with purely SVs for almost all densities of AVs.

\section{Decision-Tree Based Function Approximation}

The decision-tree based experiment was executed using XGBoost, where the number of rounds of boosting was increased gradually from 50 to 200, the evaluation criteria was the mean squared error, and the learning rate was 0.3. In the XGBoost experiment results, displayed in Figure 5.4, RF5 performed extremely poorly overall, and so it was removed from the results displayed. Otherwise, the overall speeds were slower than the linear regression results, though the trends generally remained the same.

\subsubsection{Deep Q Networks}

The final RL framework tested for single lane roads were Deep Q Networks. While the same state space was used, two different network architectures were compared, 
differing in terms of the size and number of layers. Both networks used feed-forward fully-connected layers with different numbers of hidden neurons, with Rectified Linear Unit activation functions, a mean squared error loss function and the Adam Optimizer, and were trained for 10 epochs using a batch size of 100 on every iteration. Furthermore, all networks were trained with a discount factor of 0.1 , that steadily increased until 0.5 , and then was held constant until convergence. The learning rate began at 0.5 , was steadily decreased until it was less than 0.05 , and thereafter was also held constant. Note that there are no eligibility trace mechanisms that are feasible in this scenario, and this holds for most Deep Q Networks.

To determine which NN framework was the most optimal one, the networks were first cross-validated by testing their ability to predict the expected reward given the input features, after the exploration phase. The network architectures were tested by using hidden layers, each of which had sizes equalling the number of features present in the modelling framework, and additionally 100, 500, and 1000 units. The number of layers were tested for values from 1 to 10 . The results presented here were the best obtained, and subsequently applied to the DQN architecture.

The first experiments, with results shown in Figure 5.5, were performed with a NN with 3 hidden layers, each containing 14 hidden units, and each separated by one dropout layer with a rate of 0.5 . As can be seen, the results were not particularly good, especially for RF2, RF4, and RF5, and only RF1 maintained a consistently higher average road speed for all percentages of AVs.

These results improved, as shown in Figure 5.6, by using a NN constructed with 2 hidden layers of 1000 units each after the input layer, each separated with one dropout layers with a rate of 0.5 , which were then fed to a hidden layer of 14 units prior to the output layer. As can be seen, the second network architecture yielded the best results seen so far in this section, especially with lower proportions of AVs. From the results, it appears in this scenario that RF5 performed the best, followed closely by RF2 and RF4. RF1 and RF3 still performed relatively poorly, but they continued to outperform the stochastic experiment results for the most part. It is very interesting to observe that with only $10 \%$ of the road converted to autonomous vehicles, the resulting road speed could be anywhere from $5 \%$ to $26 \%$ closer to the maximum 
Starting Speeds of DQN SL Expers

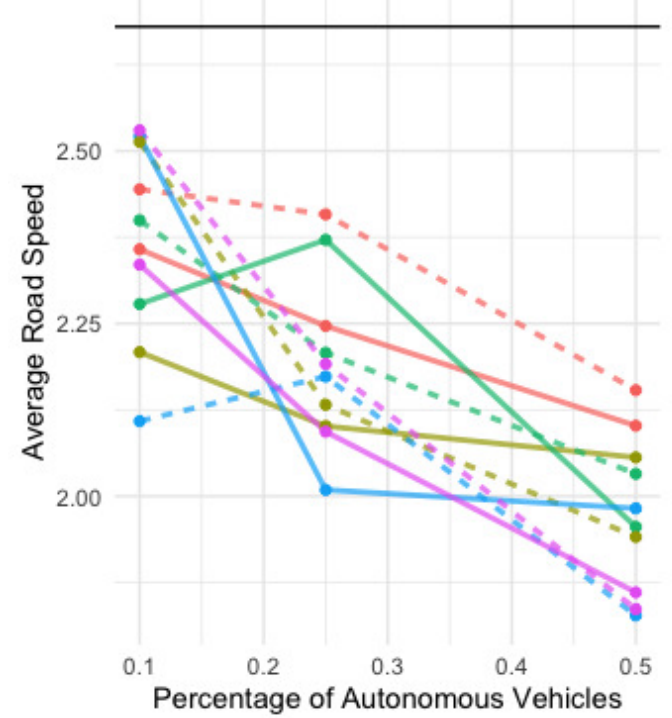

End of Experiment With Small Network

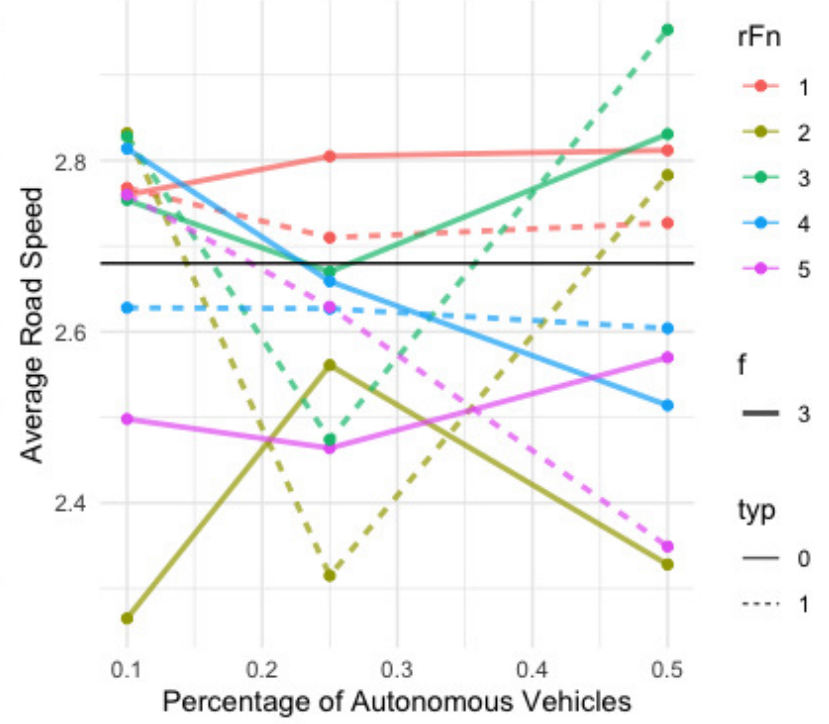

Figure 5.5: The exploration phase (left) and convergence (right) Average Speeds for Deep Q-Network Based Single Lane RL experiments, trained with a smaller NN, including the SV convergence value (horizontal black line).

\section{Starting Speeds of DQN SL Expers}

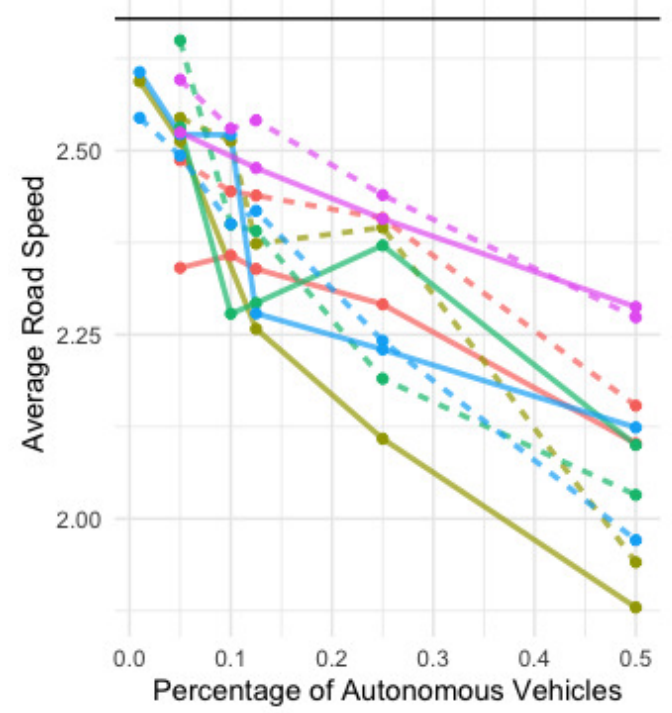

\section{End of Experiment With Large Network}

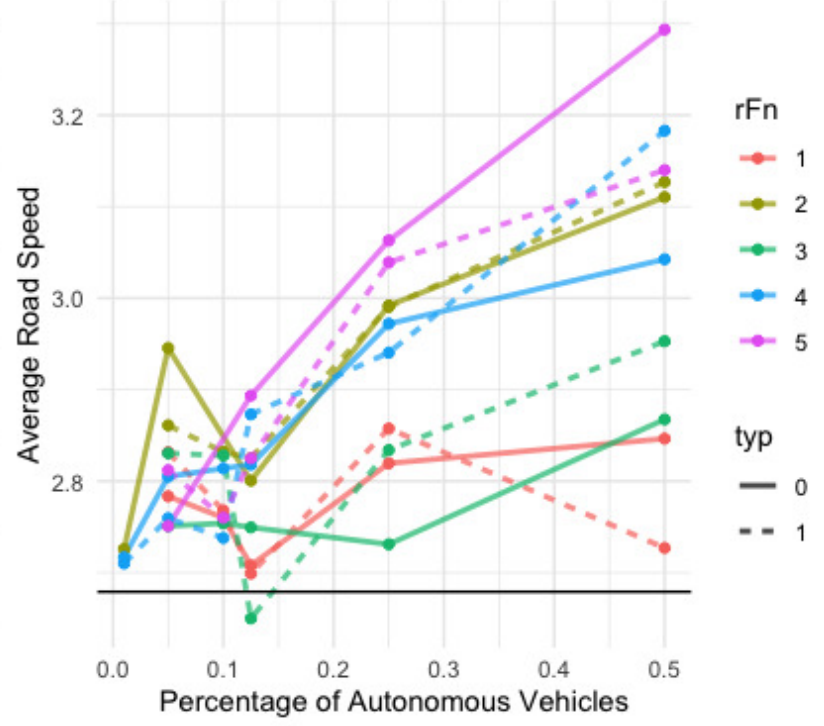

Figure 5.6: The exploration phase (left) and convergence (right) Average Speeds for Deep Q-Network Based Single Lane RL experiments, trained with a larger NN, including the SV convergence value (horizontal black line). 


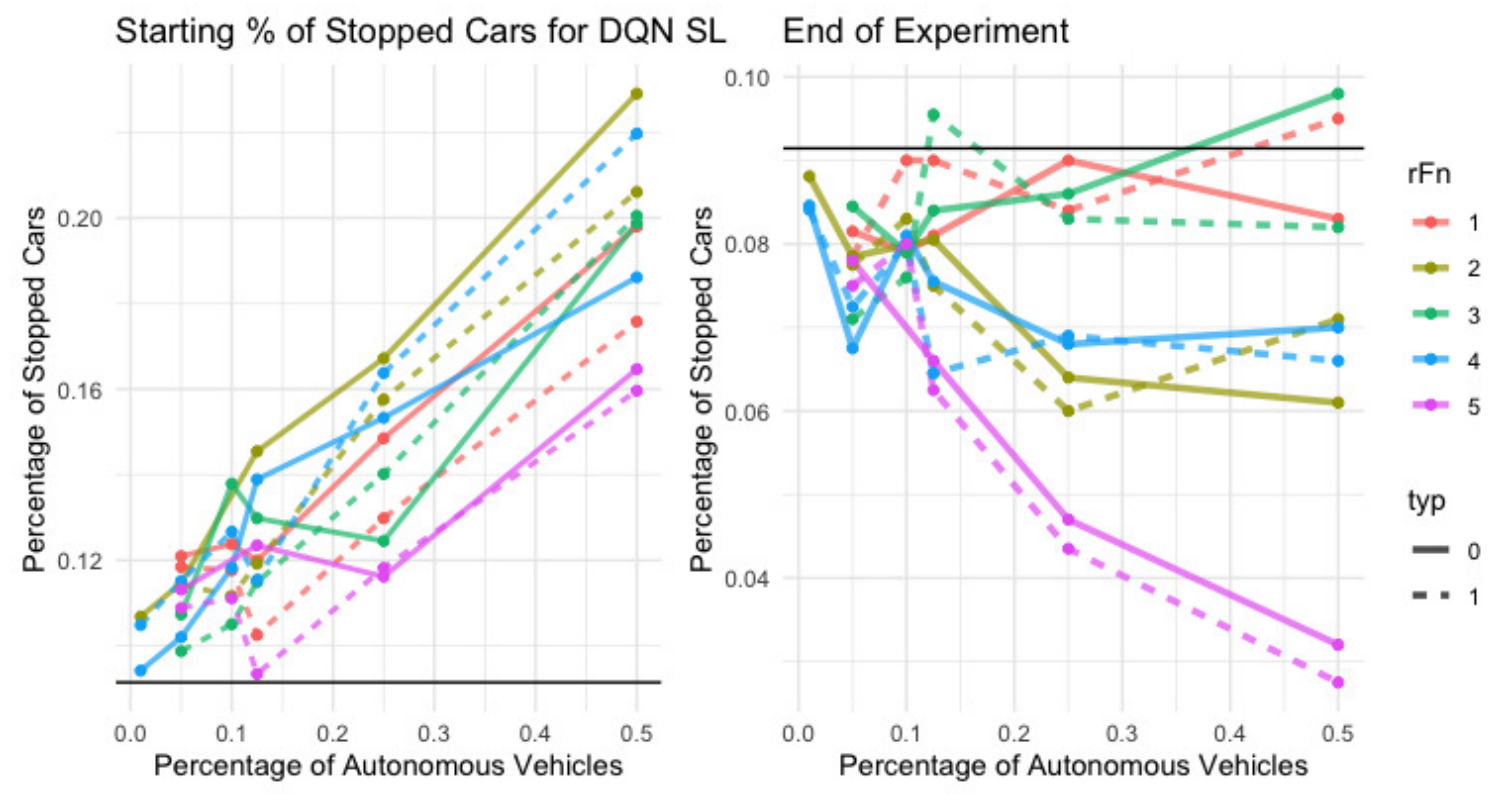

Figure 5.7: The exploration phase (left) and convergence (right) Average Percent of Stopped Cars for Deep Q-Network Based Single Lane Experiments, trained with a larger NN, including the SV convergence value (horizontal black line).

possible theoretical speed of the road. It is also hard to clearly distinguish whether or not the initial orientation of the AVs rendered a large impact on overall speed, as this seems more dependent generally on the reward function and the percentage of AVs.

Looking at the stopped car behaviour in Figure 5.7, it appears that in the experiments which performed better, the total number of stopped cars was extremely low proportional to how much the average speed of the road increased. Additionally, as seen in Figure 5.8, the average absolute change in speed per iteration also dropped noticeably for RF5, and slightly for RF2 and RF4. This suggests that, overall, all cars in the road were not being impacted as frequently by the stop-and-go waves that occurred in experiments with only SVs. This trend continued growing once larger proportions of the road were converted to AVs. Finally, it was observed in the experiments, especially with higher percentages of AVs, that the AVs attained to a slower average speed than the SVs on the road, suggesting that the increase in the overall road speed was due to the AVs managing their gaps with each other, and with the 


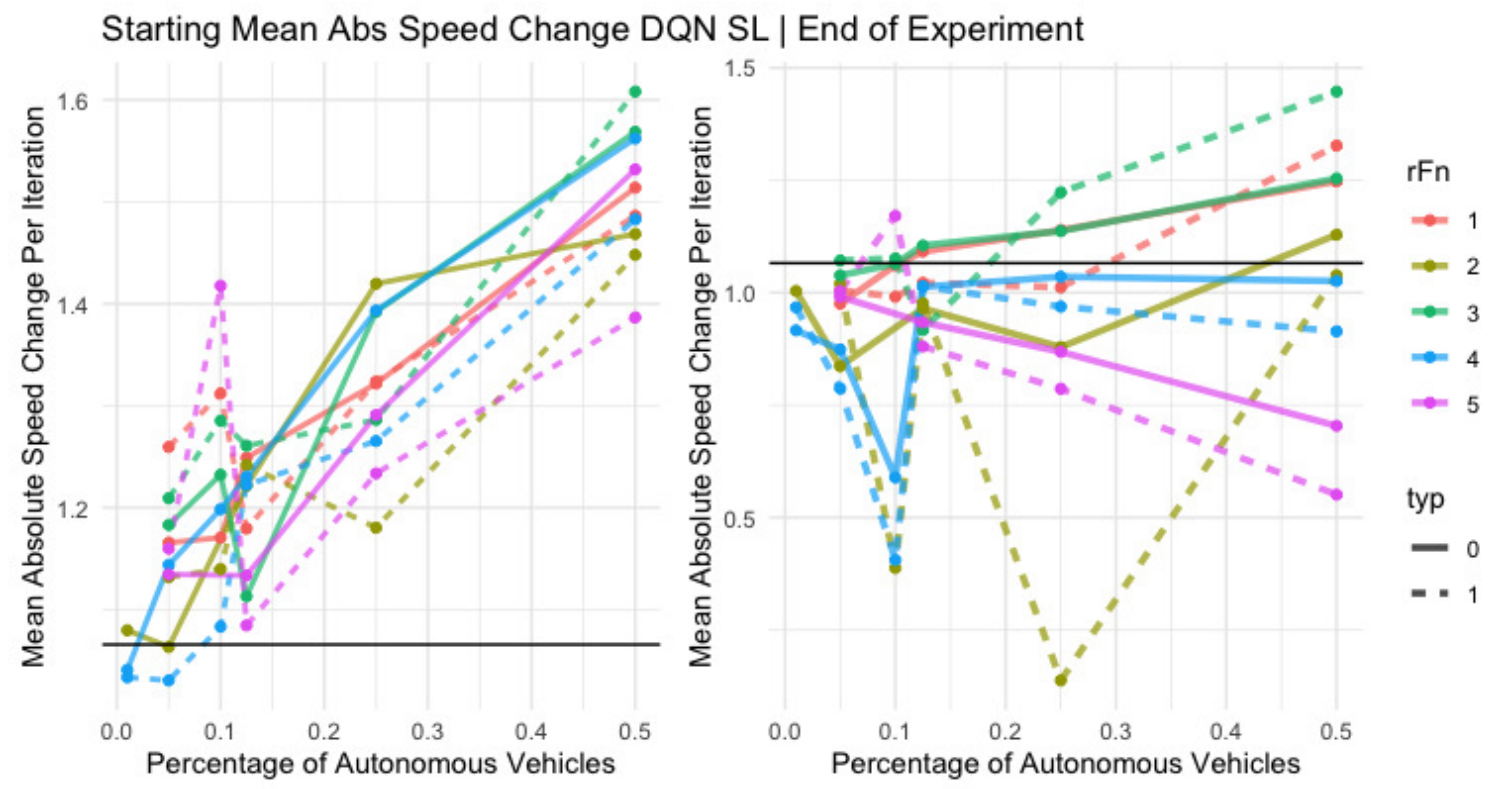

Figure 5.8: The exploration phase, or starting (left) and convergence, or finishing (right) Mean Absolute Change in Speed per Iteration for Deep Q-Network Based Single Lane Experiments, trained with a larger NN, including the SV convergence value (horizontal black line). 
SVs on the road.

Clearly, the use of RL to train AVs for road navigation yielded a noticeable improvement in terms of increasing the average speed and decreasing the percentage of cars that were forced to stop. Therefore, it can be asserted that the first approach to mitigating double lane road traffic is to place AVs that do not change lanes on a road with SVs that do, and the results of this conjecture are covered in the next section.

\subsection{Single Lane AVs in Double Lane Experiments}

This section specifically focuses on using AVs that only make speed decisions and therefore stay on the same lane throughout the experiments, while the SVs follow the multi-lane rules from Chapter 4. First, the new state space and network architectures will be specified, followed by results for both closed and open roads.

\subsubsection{State Space and Network Architecture}

The state space for the single lane NNs in these experiments included all elements from the single-lane state space, as well as the Front and Back Gaps in the other lane, the preference factor on the road cell the car is on in the current cell, and the preference factor for the same cell in the other lane. This created a state space with 17 features. The car continued to maintain the existing action space, specifically choosing a speed less than the Front Gap and the Maximum Speed of the road cell it was on.

The NN used in this scenario had 3 layers, each with 500 hidden units and a Rectified Linear Unit action function, with no dropout layers, and concluded with a linear output unit. The rest of the architecture was the same as in the single-lane case, including the discount factor and exploration rate. This NN structure was also chosen experimentally as the best, also obtained after tuning the various parameters involved. The output and target $\mathrm{Q}$ networks were trained with the same architecture, and the target $\mathrm{Q}$ network was replaced every 30 iterations. The exploration rate, $\epsilon$, for these experiments, was eventually held fixed at 0.05 , and therefore, even after a 


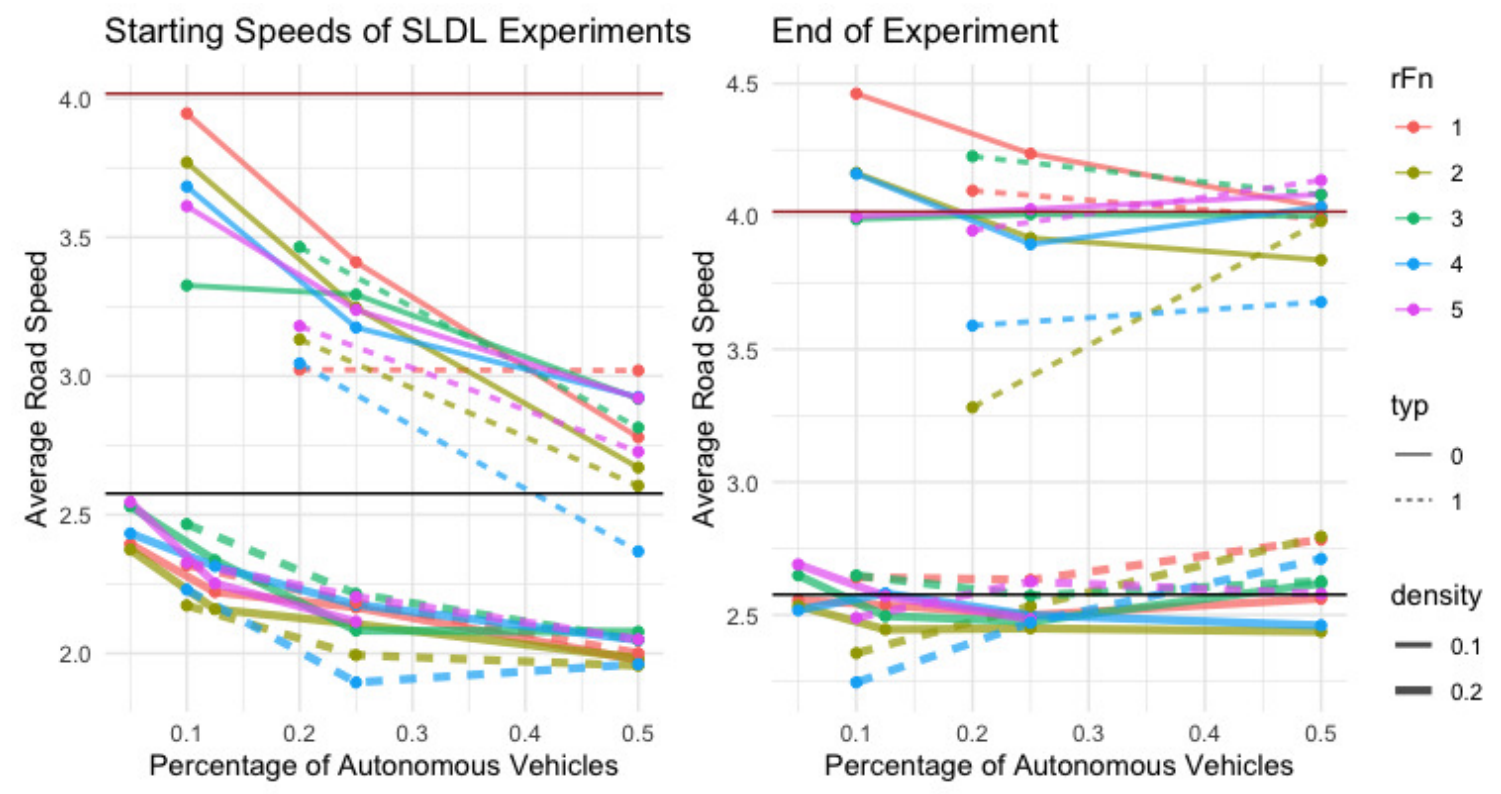

Figure 5.9: Starting and finishing stopping percentages for NN-based single lane RL experiments on sparse and dense double lane roads, including the convergence values of the fully SV experiments (red for sparse roads, and black for dense roads).

general convergence there was still the possibility of variability.

In all the graphs for double-lane experiments, sparse and dense roads are plotted on the same graph, but, typically, the convergence speeds and benchmarks are different enough that the lines do not intersect.

\subsubsection{Results and Analysis}

The results shown in Figure 5.9 show the average road speed of the experiments at the start, or the exploration phase, and finish, or the convergence phase. As is visible in the double lane setting, cars initially slowed down, much more drastically in the sparse road settings compared to the dense road settings For the most part, all reward functions appear to behave similarly, with a slightly more noticeable slowdown for RF2 and RF4. However, it can be seen at the end of the experiment, that in the sparse road settings, shown at the top half of the graph in Figure 5.9 on the right, there was not a noticeable improvement in general as the number of AVs increases. 

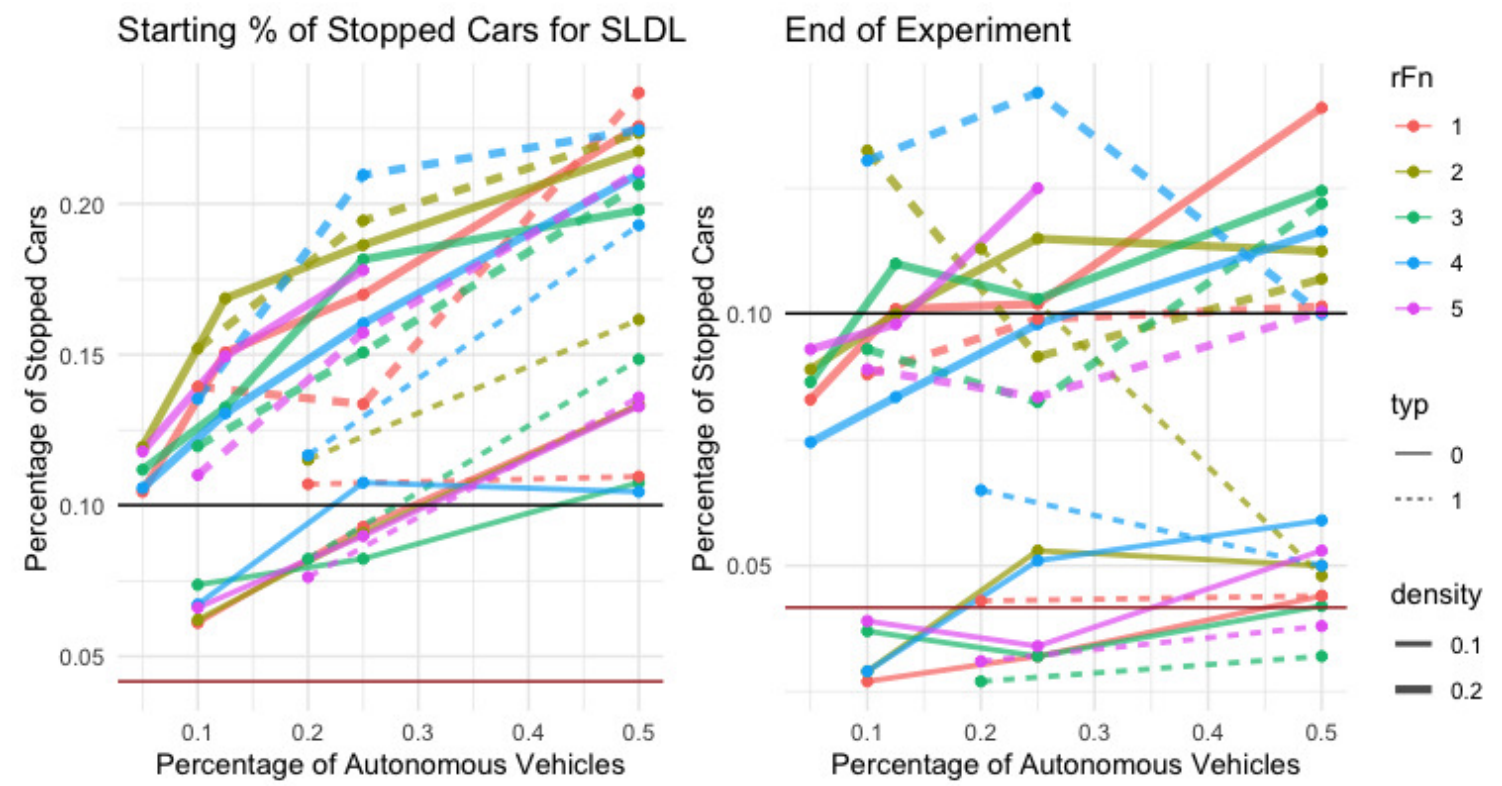

Figure 5.10: Starting and finishing stopping percentages for NN-based single lane RL experiments on sparse and dense double lane roads, including the convergence values of the fully SV experiments (red for sparse roads, and black for dense roads).

It is interesting that in the sparse road settings, for smaller numbers of AVs, RF1 appeared to perform well, but in larger numbers, this trend generally did not continue, and therefore the results were not particularly significant. On the other hand, the dense road setting showed a marginal improvement as the percentage of AVs increased, especially in the Type 1 orientation, but overall the improvement was not nearly as significant as in the single lane road experiments.

The stopping percentage graphs at the end of the experiment, shown in Figure 5.10 , behaved as expected at the start of the experiment, but were extremely inconsistent and filled with variability at the end of the experiment, especially for the dense road scenario. For the sparse road scenario, the percentage of stopped cars generally got larger as the number of AVs increased, but this appeared to be because the AVs were behaving greedily and therefore leaving uneven gaps. Therefore, overall, single lane AVs did not improve road conditions and mitigate the emergent jams as well as what was expected.

The convergence results for open road experiments are displayed in Figure 5.11. 


\section{SLDL Open Road Avg Speeds}

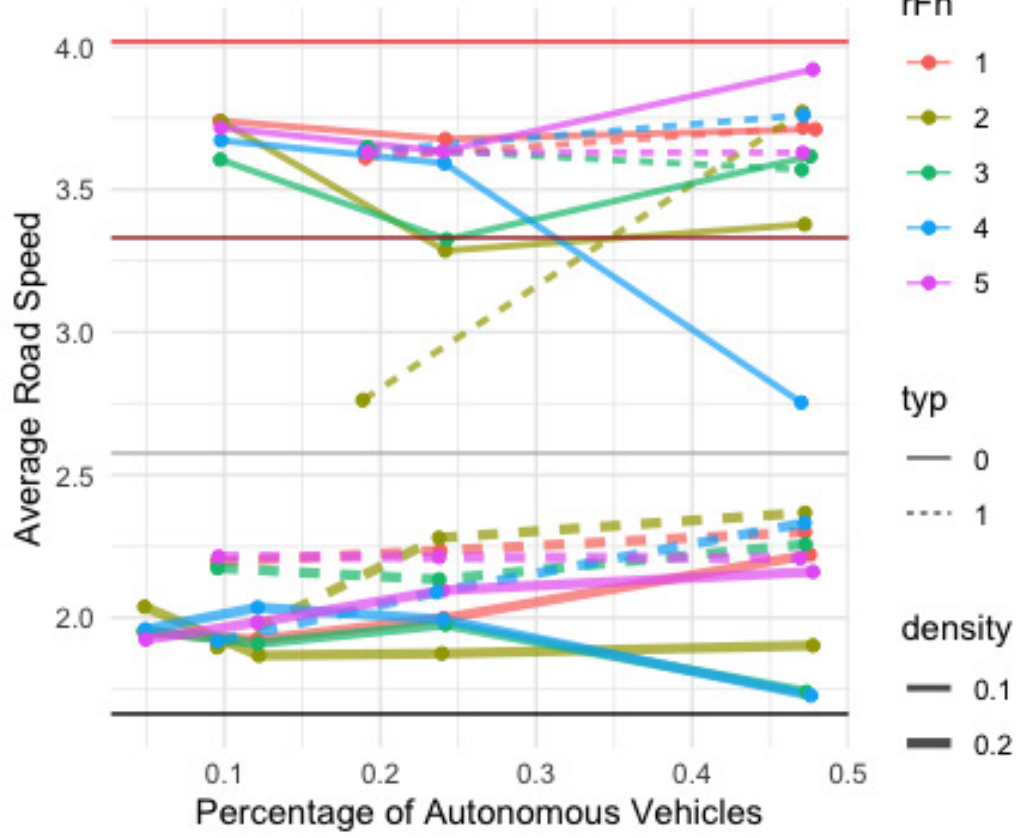

Figure 5.11: Convergence average speeds for NN-based single lane RL experiments on sparse and dense double lane open roads, including convergence values for both closed and open roads (grey/black for dense roads, and brown/red for sparse roads). 
Because the stochastic vehicles did especially poorly with the addition of entries and exits in multi-lane roads, both the closed and open road benchmarks are plotted for sparse (in red/brown, respectively) and dense (in grey/black, respectively) roads. As can be seen, even single lane vehicles in multi-lane settings out-performed the benchmarks for dense roads, and most of the reward functions outperformed the benchmarks for sparse roads. Neither of them were able to bring the speed up to the benchmark of the closed road settings in open roads. This would be the ideal situation, because it would suggest that cars were not impacted by the entry of new vehicles, which is a major factor in efficient roadways. One reason why larger percentages of $\mathrm{AVs}$ were, by default, more likely to outperform roads with lower percentages, was because AVs do not enter or exit the road. Therefore, based on the algorithms used for entries and exits, presented in Section 4.2, fewer cars will enter or exit on each iteration, meaning the random speed with which a car enters is not as likely to reduce the average speed of the overall road. Still, this particular result is a remarkable improvement!

The results presented in this section show that when translating single lane AVs to multi-lane scenarios, closed roads traffic is marginally improved, while, on the other hand, open roads benefit greatly. Therefore, it was worth investigating whether or not AVs that change lanes can further improve multi-lane road traffic as well.

\subsection{AVs and Lane Changing}

The challenge with AVs that are able to change lanes on double lane roads is that the action space is enlarged; furthermore, there are many constraints which limit the action space in the adjacent lane, on each iteration. This means that much more exploration is required for the vehicles to understand their state-to-state transitions. Similar to the previous section, this section begins by explaining the new state space and network architecture, and follows with results in closed and open roads. 

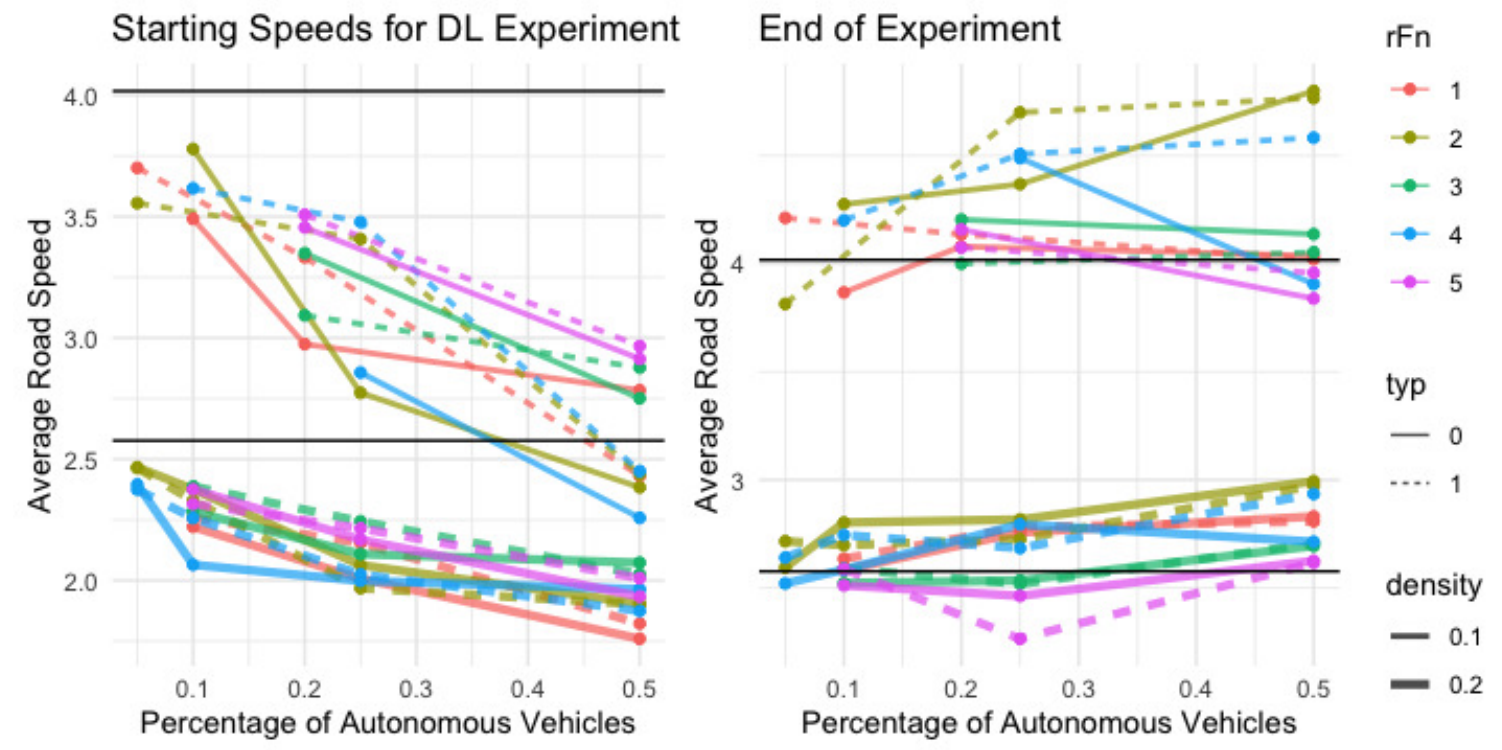

Figure 5.12: Starting and finishing stopping percentages for NN-based double lane RL experiments on double lane roads.

\subsubsection{State Space and Network Architecture}

The previous experiments with single-lane AVs in double-lane environments involved a state space of 17 elements. In experiments where AVs are able to change lanes, the state space is augmented by including the previous three Back Gaps and Front Gaps in the other lane instead of just the current Back and Front Gaps. Additionally, it also includes the lane that the AV selected during the previous iteration, which can now be different than the current lane that the AV is on. This creates a set of 24 features. The same network architecture, described in Section 5.4.1, except with an additional layer containing 10 hidden units prior to the linear output layer, was used.

\subsubsection{Results and Analysis}

The first graphs, shown in Figure 5.12, present the results for both sparse and dense closed roads. As can be seen, RF2 and RF4 yielded the best convergence by the end of the experiment, especially starting in Type 1 orientations. For dense road settings, RF1 also performs well. The improvements in Sparse Roads were much 


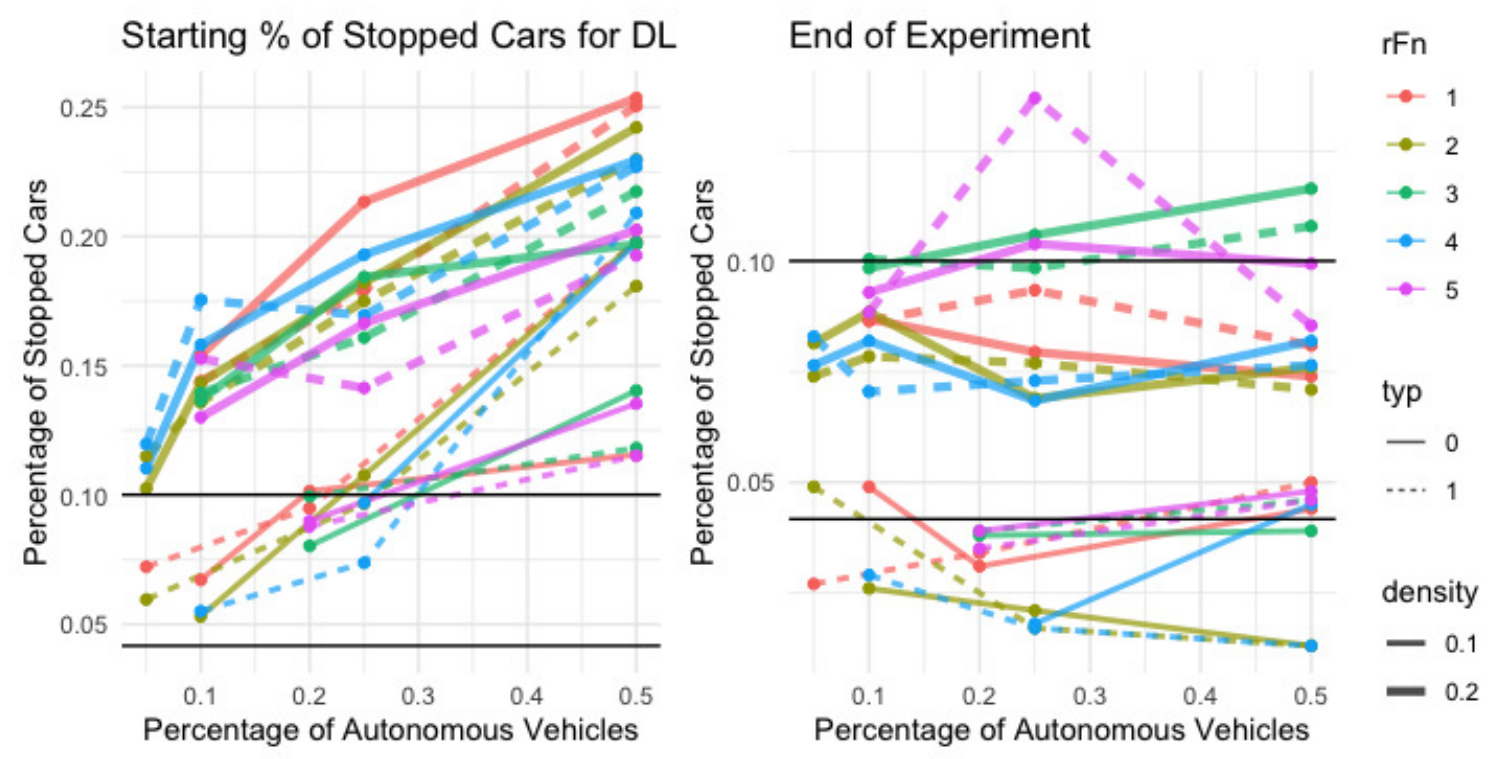

Figure 5.13: Starting and finishing stopping percentages for NN-based double lane RL experiments on double lane roads.

more significant than for Dense roads, but in both, these performed much better than the single lane AVs placed in double lane roads.

Looking at the percentage of stopped cars, in Figure 5.13, it is evident that both for dense and sparse roads, RF2 and RF4 demonstrated a consistently significant impact on decreasing the total percentage of cars which were stopped, so long as at least 10 percent of cars were AVs. Just 10 percent of cars being AVs reduced the number of stopped cars by around 25 percent in dense roads, and by around 40 percent in sparse roads.

By incorporating entries and exits, the results for all 5 reward functions were much more consistent than above, and in Type 1 orientation, are noticeably better in roads with lower percentages of AVs, especially in the sparse road settings, as shown in the top half of Figure 5.14. However, especially in dense open roads, the single lane AVs trained in double lane environments generally performed better than AVs which were able to change lanes, as seen in the bottom half of Figure 5.14, though both outperformed the SV experiments consistently. 


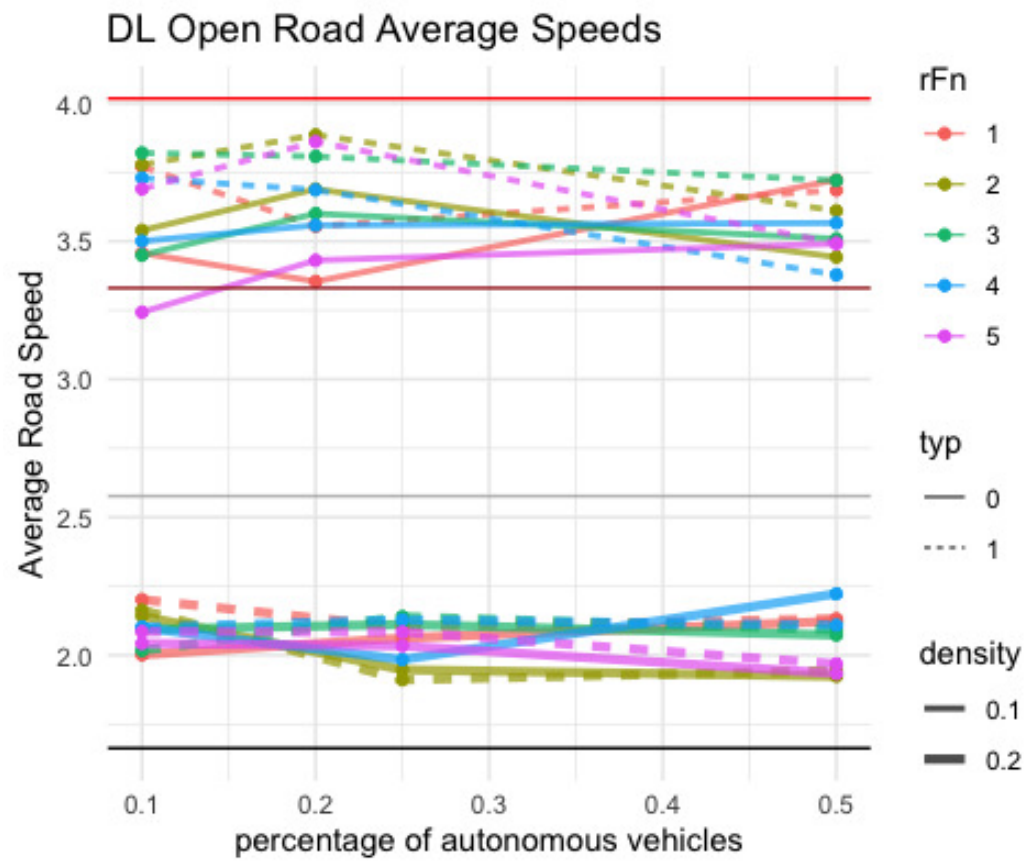

Figure 5.14: Finishing Average speed for NN-based double lane RL experiments on double lane open roads.

\subsection{Main Results}

For each road type experimented with, first the modelling framework that produced the best results, followed by statistics and interpretations of the way mixed-autonomy roads differed from stochastic road simulations are presented:

1. Closed Single Lane Dense Roads:

(a) The large Deep Q Networks, referenced in Section 5.3.3, provided the best RL framework to improve on this road type.

(b) With only $5 \%$ of the road converted to AVs, the average speed was increased by $10 \%$, the percentage of stopped cars was decreased by $14 \%$, and the mean absolute speed change was decreased by $21 \%$.

(c) With $50 \%$ of the road converted to AVs, the average road speed was increased by $23 \%$, the percentage of stopped cars was decreased by $65 \%$, and the mean absolute speed change was decreased by $34 \%$. 
(d) Therefore, even with low percentages of AVs, the impact on road speed was immediately noticeable, and this effect was amplified with higher percentages of AVs on the road, especially in terms of reducing the total number of cars which were stopped, on average, on each iteration.

2. Closed Double Lane Roads:

(a) The AVs able to change lanes, referenced in Section 5.5, were the best RL framework for all closed roads.

(b) Sparse Roads:

i. With $5 \%$ of the road converted to AVs, the average speed was increased by $5 \%$, the percentage of stopped cars was decreased by $36 \%$, and the mean absolute speed change was increased by $28 \%$.

ii. With $50 \%$ of the road converted to AVs, the average road speed was increased by $19 \%$, the percentage of stopped cars was decreased by $69 \%$, and the mean absolute speed change was increased by $143 \%$.

iii. Therefore, with low percentages of AVs, the impact on road speed was not as noticeable. With higher percentages of AVs on the road, the road became extremely close to attaining the theoretical maximum possible speed, and the percentage of stopped cars decreased greatly. However, the number of speed changes per iteration on average increased greatly, suggesting that there were not as many lane changes available.

(c) Dense Roads:

i. With $5 \%$ of the road converted to AVs, the average speed was increased by $5 \%$, the percentage of stopped cars was decreased by $26 \%$, and the mean absolute speed change was increased by less than $1 \%$.

ii. With $50 \%$ of the road converted to $\mathrm{AVs}$, the average road speed was increased by $16 \%$, the percentage of stopped cars was decreased by $24 \%$, and the mean absolute speed change was increased by $33 \%$. 
iii. Therefore, with low percentages of AVs, the impact on road speed was not as noticeable compared to when higher percentages of AVs were the road. However, the percentage of stopped cars did not change significantly, and the number of speed changes increased greatly, suggesting that in this framework, drivers were constantly forced to continuously change speeds.

3. Open Double Lane Roads:

(a) The single AVs in double lane roads, referenced in Section 5.4, were the best RL framework for all open roads.

(b) Sparse Roads:

i. With $10 \%$ of the road converted to AVs, the average speed was increased by $12 \%$, the percentage of stopped cars was decreased by $30 \%$, and the mean absolute speed change was decreased by $7 \%$.

ii. With $50 \%$ of the road converted to AVs, the average road speed was increased by $18 \%$, the percentage of stopped cars was decreased by $20 \%$, and the mean absolute speed change was increased by $1 \%$.

iii. Therefore, with both low and high percentages of AVs, the impact of road speed is fairly noticeable, and especially with lower percentages of AVs, the total percentage of stopped cars decreased substantially. However, because higher percentages of AVs do not significantly improve road conditions compared to lower percentages, this suggests that even though the SV benchmark was surpassed, there was still significant room for improvement in this particular experiment type.

(c) Dense Roads:

i. With $5 \%$ of the road converted to AVs, the average speed was increased by $24 \%$, the percentage of stopped cars was decreased by $35 \%$, and the mean absolute speed change was increased by less than $1 \%$. 
ii. With $50 \%$ of the road converted to AVs, the average road speed was increased by $44 \%$, the percentage of stopped cars was decreased by $68 \%$, and the mean absolute speed change was increased by $80 \%$.

iii. Therefore, with both low percentages of AVs, the impact of road speed is fairly noticeable, and this is further amplified with higher percentages of AVs, especially when it comes to decreasing the total percentage of stopped cars.

\subsection{Conclusions}

Clearly, AVs trained with RL are able to make improvements on overall road speeds compared to roads containing purely SVs. This occurred even when the number of AVs on the road is a small percentage of total vehicles. The results improved further when larger percentages of the road were converted to AVs, except for open double lane sparse roads, where the results only improve marginally as the percentage of AVs increases. Appendix A.2 contains tables holding the full list of average experiment results.

The reward functions which performed the best were functions of the speeds or gaps of all AVs on the road, which suggests that the underlying Q networks learned, to some extent, how their current actions had downstream impacts on other vehicles, though it is difficult to measure exactly to what extent. The improvement in road speed is primarily because of the ability of AVs to manage their gaps better, and therefore prevent the rate of occurrence of emergent traffic jams. This can also be seen, generally, by the reduction in fully stopped vehicles, and the decrease or sustenance of the mean absolute speed differences. Furthermore, these AVs did not receive information about all the cars on the road at every time instance, but instead made their decisions based on a replay buffer filled with the same local factors that SVs used to make decisions, and only had the competitive advantage of being able to recall past experiences, and share experiences with other AVs on the road.

As mentioned in Chapter 2, there was no work in the previous literature directly comparable to what was done in the thesis. However, these results appeared to 
follow similar patterns as those presented in [20], with less extreme results due to the comparably-reduced state space. 


\section{6 Conclusions and Future Research}

In this chapter, we will first provide a summary of the thesis, followed by a brief review of the main results. Finally, we will conclude the thesis by stating a few of the many avenues of future work and research.

\subsection{Summary}

The objective of this thesis was to assess the impact of Autonomous Vehicles (AVs) on improving traffic flow in mixed-autonomy roads. AVs were trained with state-ofthe-art Machine Learning techniques, specifically Deep Reinforcement Learning (RL), designed to maximize overall road speeds and mitigate emergent jams. However, they were only given the minimum information required to make safe decisions. Understanding the dynamics of emergent traffic jams in mixed-autonomy road systems, which are extremely complex given the wide range of driving patterns exhibited by 
humans, is generally an overlooked aspect in the growing automation of cars. After all, if the addition of even a few AVs, with only the basic input features, helps reduce the total amount of time humans spend sitting in traffic, and therefore in reducing the overall economic cost of traffic, it will be much easier to eventually transition to highways that are completely filled with AVs, which are, by far, the most efficient and safest long-term solution for transportation networks.

In Chapter 2, we surveyed the fields for the three distinct areas of research related to this thesis: collision-free road traffic modelling, model-free RL, and methods for training AVs to navigate road traffic using RL. We first analyzed micro-traffic road modelling, specifically the Nagel-Schreckenberg modelling framework [10], including analyzing the parallels between previous work showing the advantages of communication between vehicles, and the potential advantages of AVs. Next, we reviewed model-free RL, focused on Q learning, which was used to design the AVs in the thesis. Finally, we reviewed many related works that used RL to train AVs, and therefore motivated the solutions used in this thesis.

In Chapter 3, we defined the road modelling framework that was used for all traffic experiments in the thesis. This road modelling was designed to track the lane and speed of every car on every iteration, where each iteration represented one time interval on the road (i.e., one second). The chapter began with a detailed description of the representations of both the road tensor and the car matrix, and algorithms for how the road was updated on each iteration. This included algorithms for how cars traversed, entered, and exited the road, and the logic for all the different features of the road that impacted the interactions between the cars. Finally, we cataloged the specific criteria that were used to assess the conditions of the road.

In Chapter 4, we presented the algorithms used for simulating the speed and lane decisions of human drivers, referred to as Stochastic Vehicles (SVs). These algorithms combined a rule-set inspired from the literature review on NS traffic modelling, but with a larger action space that promoted more diverse and aggressive behaviour, and also including stochasticity. Furthermore, experiments were run for sparse and dense single lane roads with no entering or exiting cars, and sparse and dense double lane 
roads both with and without entries and exits. The cars averaged the theoretically optimal road speed for sparse single lane roads, but for all dense single lane experiments, and most double lane experiments, the roads did not attain the theoretically-optimal speeds. Furthermore, we established a benchmark of traffic conditions for the experiments based on criteria given in Chapter 3, so as to obtain an idea of the prevalence of traffic jams on roads containing only human drivers.

In Chapter 5, we developed the AVs, using RL. This involved testing a variety of RL approaches on single lane roads, and taking the best approach from the group, deep Q networks, to be used for double lane setting. Furthermore, single lane AVs were also tested on double lane roads. As it turns out, most of the experiments exceeded the baselines set in Chapter 4, for both single and double lane roads. It was also determined that this was largely due to the overall reduction in jammed vehicles. The improvement was due to more than just the AVs individually performing better. Rather, in most experiments, AVs actually took on lower average speeds than SVs!

\subsection{Future Work}

In conclusion, the following are some of the future avenues of research that can emerge from the results in this thesis:

- Adapting the road modelling framework to work with different sized vehicles, and factoring in the time where cars which can be "in between" two lanes when changing lanes. Also, using on ramps and off ramps to simulate entries and exits, and also testing scenarios of lanes merging (lanes closing).

- Adapting this framework to city driving with traffic lights and intersection management, and running experiments on the scale of real highway networks, with extremely large NNs run on multiple GPUs.

- Addressing some of the ethical concerns related to the increased use of technology in daily life. It is important to understand that this problem is not fully realistic, because, as long as human drivers share the road, there is always the 
possibility of collisions occurring. Furthermore, it is always possible for particular drivers to identify and attempt to "trick" AVs by taking a series of malicious actions. Therefore, in the future, it will be very important to incorporate these concerns into the real-life modelling of AVs. 


\section{Bibliography}

[1] Ian Goodfellow, Yoshua Bengio, and Aaron Courville. Deep learning. MIT press, 2016.

[2] Carl-Johan Hoel, Krister Wolff, and Leo Laine. Automated speed and lane change decision making using deep reinforcement learning. In 2018 21st International Conference on Intelligent Transportation Systems (ITSC), pages 21482155. IEEE, 2018.

[3] Texas A\&M Transportation Institute. Urban mobility report. 2020.

[4] Yuka Ishihara and Toshiharu Sugawara. Analysis of traffic congestion reducer agents on multi-lane highway. In 2019 2nd International Conference on Intelligent Autonomous Systems (ICoIAS), pages 135-141. IEEE, 2019.

[5] Shota Ishikawa and Sachiyo Arai. Evaluating advantage of sharing information among vehicles toward avoiding phantom traffic jam. In 2015 Winter Simulation Conference (WSC), pages 300-311. IEEE, 2015.

[6] Shota Ishikawa and Sachiyo Arai. Cooperative learning of a driving strategy to suppress phantom traffic jams. In 2016 IEEE International Conference on Agents (ICA), pages 90-93. IEEE, 2016.

[7] Harshal Maske, Tianshu Chu, and Uroš Kalabić. Large-scale traffic control using autonomous vehicles and decentralized deep reinforcement learning. In 2019 
IEEE Intelligent Transportation Systems Conference (ITSC), pages 3816-3821. IEEE, 2019.

[8] Volodymyr Mnih, Koray Kavukcuoglu, David Silver, Andrei A Rusu, Joel Veness, Marc G Bellemare, Alex Graves, Martin Riedmiller, Andreas K Fidjeland, Georg Ostrovski, et al. Human-level control through deep reinforcement learning. Nature, 518(7540):529-533, 2015.

[9] David E Moriarty and Pat Langley. Learning cooperative lane selection strategies for highways. AAAI/IAAI, 1998:684-691, 1998.

[10] Kai Nagel and Maya Paczuski. Emergent traffic jams. Physical Review E, 51(4):2909, 1995.

[11] Kai Nagel and Michael Schreckenberg. A cellular automaton model for freeway traffic. Journal de physique I, 2(12):2221-2229, 1992.

[12] Subramanya Nageshrao, H Eric Tseng, and Dimitar Filev. Autonomous highway driving using deep reinforcement learning. In 2019 IEEE International Conference on Systems, Man and Cybernetics (SMC), pages 2326-2331. IEEE, 2019.

[13] Toronto Region Board of Trade. Getting ready for autonomy: Avs for safe, clean and inclusive mobility in the toronto region. 2020.

[14] Marcus Rickert, Kai Nagel, Michael Schreckenberg, and Andreas Latour. Two lane traffic simulations using cellular automata. Physica A: Statistical Mechanics and its Applications, 231(4):534-550, 1996.

[15] Richard S Sutton and Andrew G Barto. Reinforcement learning: An introduction. MIT press, 2018.

[16] Martin Treiber, Ansgar Hennecke, and Dirk Helbing. Congested traffic states in empirical observations and microscopic simulations. Physical review E, $62(2): 1805,2000$. 
[17] Hado Van Hasselt, Arthur Guez, and David Silver. Deep reinforcement learning with double q-learning. In Thirtieth AAAI conference on artificial intelligence, 2016.

[18] Guan Wang, Jianming $\mathrm{Hu}$, Zhiheng Li, and Li Li. Cooperative lane changing via deep reinforcement learning. arXiv preprint arXiv:1906.08662, 2019.

[19] Ziyu Wang, Tom Schaul, Matteo Hessel, Hado Van Hasselt, Marc Lanctot, and Nando De Freitas. Dueling network architectures for deep reinforcement learning. arXiv preprint arXiv:1511.06581, 2015.

[20] Cathy Wu, Aboudy Kreidieh, Eugene Vinitsky, and Alexandre M Bayen. Emergent behaviors in mixed-autonomy traffic. In Conference on Robot Learning, pages 398-407, 2017.

[21] Chao Yu, Xin Wang, Xin Xu, Minjie Zhang, Hongwei Ge, Jiankang Ren, Liang Sun, Bingcai Chen, and Guozhen Tan. Distributed multiagent coordinated learning for autonomous driving in highways based on dynamic coordination graphs. IEEE Transactions on Intelligent Transportation Systems, 2019.

[22] Taiyi Zhang and Yu-Cheng Lin. Hybrid multilane models for highway traffic. The European Physical Journal B, 91(11):274, 2018. 
Appendices 


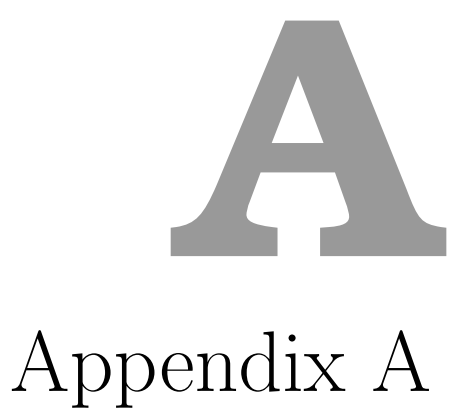

This appendix begins by giving technical specifications for how the AVs were trained. It also contains tables of results for most of the experiments in Chapter 4 and 5 of the thesis, for both SVs and AVs.

\section{A.1 Technical Specifications}

All the coding for this thesis was done using the $\mathrm{R}$ programming language, as it was efficient for processing data frames and matrices, which were required for efficiently updating the road. The dplyr package was used to execute all table processing tasks (filtering, sorting, etc). All SVs, and road updating functions, as a result, were programmed from scratch.

For tabular Q learning experiments, we employed the Watkins Q Learning algorithm. This was written "from scratch" as well because a suitable package to meet 
the requirements for the thesis was not available. Similarly, for all regression and decision-tree experiments, the inbuilt Im function, and xgboost package were used as the function approximator. Besides that, all functions were written from scratch.

All the NNs in the thesis were built using the Keras $\mathrm{R}$ package, which directly receives an $\mathrm{R}$ matrix as input, and allows for the user to manually construct and piece together the different layers, specify the number of hidden neurons, the activation function applied, the compiler and loss functions to be used, etc. The functions which interacted with the NN built in Keras were also written from scratch and developed specifically for the traffic environment.

\section{A.2 Results Tables}

The results for closed road SV experiments for dense single lane roads, and sparse and dense double lane roads, for all different car types, are in Figure A.1. Most of the results for the different percentages of AVs tested on single lane roads with different function approximators are in Figure A.2. The results for single lane AVs in double lane roads are given in Figure A.3, and the results for double lane AVs are in Figure A.4. 
Table of Closed SV Results

\begin{tabular}{|c|c|c|c|c|}
\hline $\begin{array}{c}\text { Road Lanes/Density/ } \\
\text { Alg }\end{array}$ & Car Type & Mean Speed & $\begin{array}{c}\text { Mean \% } \\
\text { Stopped }\end{array}$ & $\begin{array}{c}\text { Mean Abs } \\
\text { Sp Change }\end{array}$ \\
\hline \multirow{3}{*}{ SL Density 0.2 } & Normal & 2.89 & 0.058 & 1.04 \\
\cline { 2 - 5 } & Passive & 2.34 & 0.124 & 0.86 \\
\cline { 2 - 5 } & Aggressive & 3.00 & 0.124 & 1.39 \\
\cline { 2 - 5 } DL Density 0.1 Closed & Mixed & 2.68 & 0.094 & 1.03 \\
\hline & Normal & 4.26 & 0.026 & 0.62 \\
& Passive & 3.56 & 0.047 & 0.72 \\
& Aggressive & 4.45 & 0.042 & 0.60 \\
\hline \multirow{3}{*}{ DL Density 0.2 Closed } & Mixed & 4.03 & 0.041 & 0.69 \\
\hline & Normal & 2.80 & 0.065 & 1.22 \\
& Passive & 2.24 & 0.116 & 0.96 \\
\hline & Aggressive & 2.91 & 0.135 & 1.58 \\
\hline
\end{tabular}

Figure A.1: Closed Road SV Results for Different Car Types. 
Table of Best Single Lane Results

\begin{tabular}{|c|c|c|c|c|c|}
\hline $\begin{array}{c}\text { Road Lanes/Density/ } \\
\text { Alg }\end{array}$ & $\%$ AVs & Reward Fn & Mean Speed & $\begin{array}{l}\text { Mean \% } \\
\text { Stopped }\end{array}$ & $\begin{array}{l}\text { Mean Abs } \\
\text { Sp Change }\end{array}$ \\
\hline SL Density $0.2 \mathrm{SVs}$ & 0 & SVs & 2.68 & 0.092 & 1.06 \\
\hline \multirow{4}{*}{ SL Density 0.2 Tabular } & 5 & 1 & 2.73 & 0.088 & 1.06 \\
\hline & 12.5 & 5 & 2.84 & 0.079 & 1.09 \\
\hline & 25 & 5 & 2.95 & 0.087 & 1.02 \\
\hline & 50 & 5 & 3.17 & 0.049 & 0.91 \\
\hline \multirow{4}{*}{ SL Density 0.2 Reg } & 5 & 5 & 2.83 & 0.095 & 1.16 \\
\hline & 12.5 & 3 & 2.94 & 0.086 & 0.98 \\
\hline & 25 & 2 & 3.00 & 0.088 & 1.04 \\
\hline & 50 & 2 & 3.22 & 0.073 & 1.39 \\
\hline \multirow{4}{*}{ SL Density 0.2 XG } & 5 & 2 & 2.78 & 0.089 & 1.06 \\
\hline & 12.5 & 4 & 2.89 & 0.089 & 1.20 \\
\hline & 25 & 2 & 2.95 & 0.091 & 1.17 \\
\hline & 50 & 4 & 3.11 & 0.081 & 1.34 \\
\hline \multirow{4}{*}{ SL Density 0.2 NN } & 5 & 2 & 2.95 & 0.079 & 0.836 \\
\hline & 12.5 & 5 & 2.89 & 0.066 & 0.934 \\
\hline & 25 & 5 & 3.06 & 0.047 & 0.868 \\
\hline & 50 & 5 & 3.29 & 0.032 & 0.704 \\
\hline
\end{tabular}

Figure A.2: Single Lane Results with AVs. 
Table of Best Double Lane Results With Single Lane AVs

\begin{tabular}{|c|c|c|c|c|c|}
\hline SLDL & $\%$ AVs & Reward Fn & Mean Speed & $\begin{array}{l}\text { Mean \% } \\
\text { Stopped }\end{array}$ & $\begin{array}{c}\text { Mean Abs Sp } \\
\text { Change }\end{array}$ \\
\hline Closed Density $0.1 \mathrm{SV}$ & 0 & SVs & 4.02 & 0.042 & 0.70 \\
\hline Closed Density $0.2 \mathrm{SV}$ & 0 & SVs & 2.58 & 0.1 & 1.19 \\
\hline \multirow{4}{*}{ Closed Density 0.1} & 10 & 1 & 4.46 & 0.027 & 0.583 \\
\hline & 20 & 3 & 4.23 & 0.027 & 0.764 \\
\hline & 25 & 1 & 4.24 & 0.032 & 0.795 \\
\hline & 50 & 5 & 4.14 & 0.038 & 1.06 \\
\hline \multirow{4}{*}{ Closed Density 0.2} & 5 & 5 & 2.69 & 0.093 & 1.24 \\
\hline & 10 & 3 & 2.65 & 0.093 & 1.20 \\
\hline & 25 & 1 & 2.63 & 0.099 & 1.16 \\
\hline & 50 & 2 & 2.79 & 0.107 & 1.38 \\
\hline Open Density 0.1 SV & 0 & SVs & 3.33 & 0.075 & 0.88 \\
\hline Open Density $0.2 \mathrm{SV}$ & 0 & SVs & 1.65 & 0.45 & 0.59 \\
\hline \multirow{4}{*}{ Open Density 0.1} & 10 & 1 & 3.74 & 0.0526 & 0.82 \\
\hline & 20 & 3 & 3.65 & 0.0447 & 0.89 \\
\hline & 25 & 1 & 3.68 & 0.0650 & 0.92 \\
\hline & 50 & 5 & 3.92 & 0.0597 & 0.89 \\
\hline \multirow{4}{*}{ Open Density 0.2} & 5 & 2 & 2.04 & 0.292 & 0.63 \\
\hline & 10 & 5 & 2.21 & 0.142 & 0.65 \\
\hline & 25 & 2 & 2.28 & 0.123 & 0.66 \\
\hline & 50 & 2 & 2.37 & 0.142 & 1.06 \\
\hline
\end{tabular}

Figure A.3: Double Lane Results With Single Lane AVs. 
Table of Best Double Lane Results Overall

\begin{tabular}{|c|c|c|c|c|c|}
\hline DL & $\%$ AVs & Reward Fn & Mean Speed & $\begin{array}{l}\text { Mean \% } \\
\text { Stopped }\end{array}$ & $\begin{array}{c}\text { Mean Abs Sp } \\
\text { Change }\end{array}$ \\
\hline Closed Density $0.1 \mathrm{SV}$ & 0 & SVs & 4.02 & 0.042 & 0.70 \\
\hline Closed Density $0.2 \mathrm{SV}$ & 0 & SVs & 2.58 & 0.1 & 1.19 \\
\hline \multirow{4}{*}{ Closed Density 0.1} & 5 & 1 & 4.21 & 0.027 & 0.895 \\
\hline & 10 & 2 & 4.28 & 0.026 & 0.861 \\
\hline & 25 & 2 & 4.7 & 0.017 & 1.10 \\
\hline & 50 & 2 & 4.8 & 0.013 & 1.70 \\
\hline \multirow{4}{*}{ Closed Density 0.2} & 5 & 2 & 2.72 & 0.074 & 1.20 \\
\hline & 10 & 2 & 2.8 & 0.089 & 1.28 \\
\hline & 25 & 2 & 2.82 & 0.069 & 1.41 \\
\hline & 50 & 2 & 2.99 & 0.076 & 1.58 \\
\hline Open Density 0.1 SV & 0 & SVs & 3.33 & 0.075 & 0.88 \\
\hline Open Density $0.2 \mathrm{SV}$ & 0 & SVs & 1.65 & 0.45 & 0.59 \\
\hline \multirow{3}{*}{ Open Density 0.1} & 10 & 3 & 3.82 & 0.046 & 0.74 \\
\hline & 20 & 2 & 3.89 & 0.055 & 0.93 \\
\hline & 50 & 1 & 3.72 & 0.065 & 1.30 \\
\hline \multirow{3}{*}{ Open Density 0.2} & 10 & 1 & 2.20 & 0.143 & 0.70 \\
\hline & 20 & 3 & 2.14 & 0.165 & 0.99 \\
\hline & 50 & 4 & 2.22 & 0.154 & 1.19 \\
\hline
\end{tabular}

Figure A.4: Double Lane Results With Double Lane AVs. 


\section{B \\ Appendix B}

\section{B.1 n-Lane Simulation Results}

This section, covering $n$-Lane simulations, involves comparing 3 lane and 4 lane open road experiments ${ }^{1}$. Both open roads had 5 entries and exits, and the sparse road experiments had a target density of 0.1 , while the dense road experiments had a target density of 0.2 . Furthermore, there were 25 consecutive cells in the first lane for all experiments that were forced to have a maximum speed of 2 , simulating an external slowdown (for example from construction), and cars were perturbed every 30 iterations, in the same method that cars in the single lane case were perturbed.

It appears that for both 3 and 4 lanes for sparse roads, the average speed converged to around 3.5, as seen in Figure B.1. This suggests that the random slowdown did

\footnotetext{
${ }^{1}$ Numerous experiments were done, but the experiments plotted in this section are just a summary of a few relevant results to give the reader an impression of how adding additional lanes changes traffic dynamics
} 

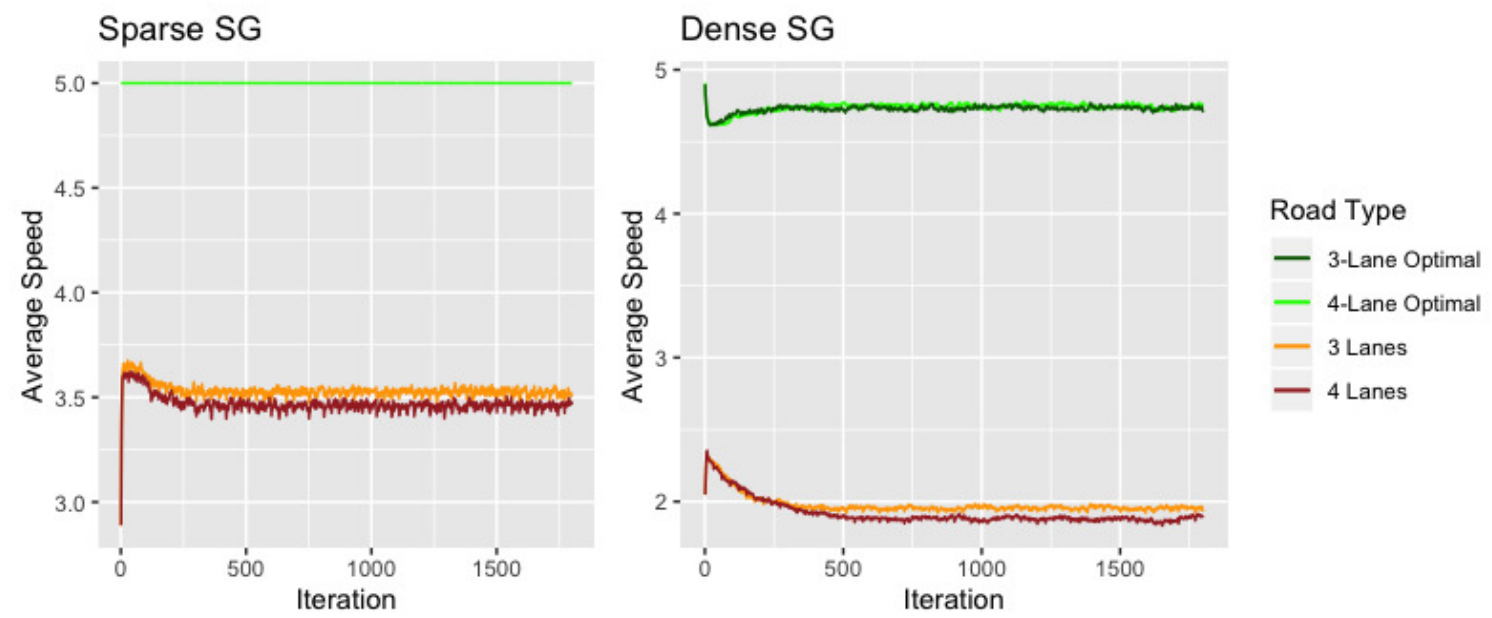

Figure B.1: Speed Graphs for Sparse (left) and Dense (right) 3-lane and 4-lane Open Road Experiments, including one forced slowdown to the maximum speed of 2 for 25 road cells at the beginning of the first lane, and also with random perturbations.

not slow the whole road down to an average speed under 2. For the dense roads, on the other hand, both 3 and 4 lane roads appeared to converge at an average speed just under 2, suggesting that the slowdown might have played a role in determining the average speed. It does appear, overall, that regardless of the forced slowdown, both 3 and 4 lane open roads were faster than the 2 lane open road!

Looking at the Change-Graph in Figure B.2, in the sparse roads, the 3 lane road had 40 percent of cars in the first lane, and also maintained a relatively low percentage of stopped cars. This suggests that cars were well balanced on all 3 lanes. The 4-lane graph, on the right, maintained a percentage above 30 percent, while also maintaining a low percentage of stopped cars, but a higher percentage of grouped jams. Overall the behaviour in sparse open roads appeared to be well balanced!

Comparing this to the Dynamics-Graphs in Figure B.3, in the dense roads, both 3 lane and 4 lane roads had an extremely high percentage of cars in the first lane, and clearly, these cars struggled to find space to make lane changes, resulting in a high percentage of cars with a large Front Gap, and cars that were stopped. Similarly, in the 4-lane dense road experiment, almost half the cars were in the first lane, which suggests that the remaining 3 lanes were actually filled with sparse traffic. 

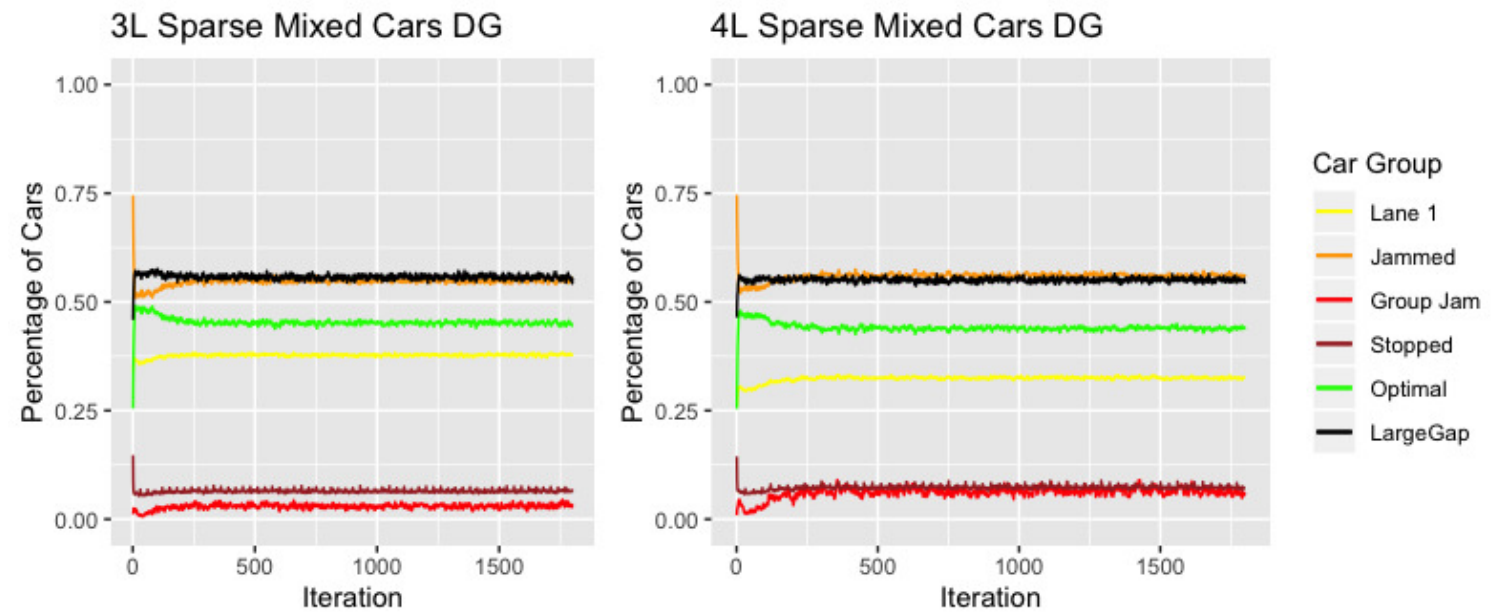

Figure B.2: Dynamics-Graph for the 3 lane (left) and 4 lane (right) Sparse Open Road Experiments
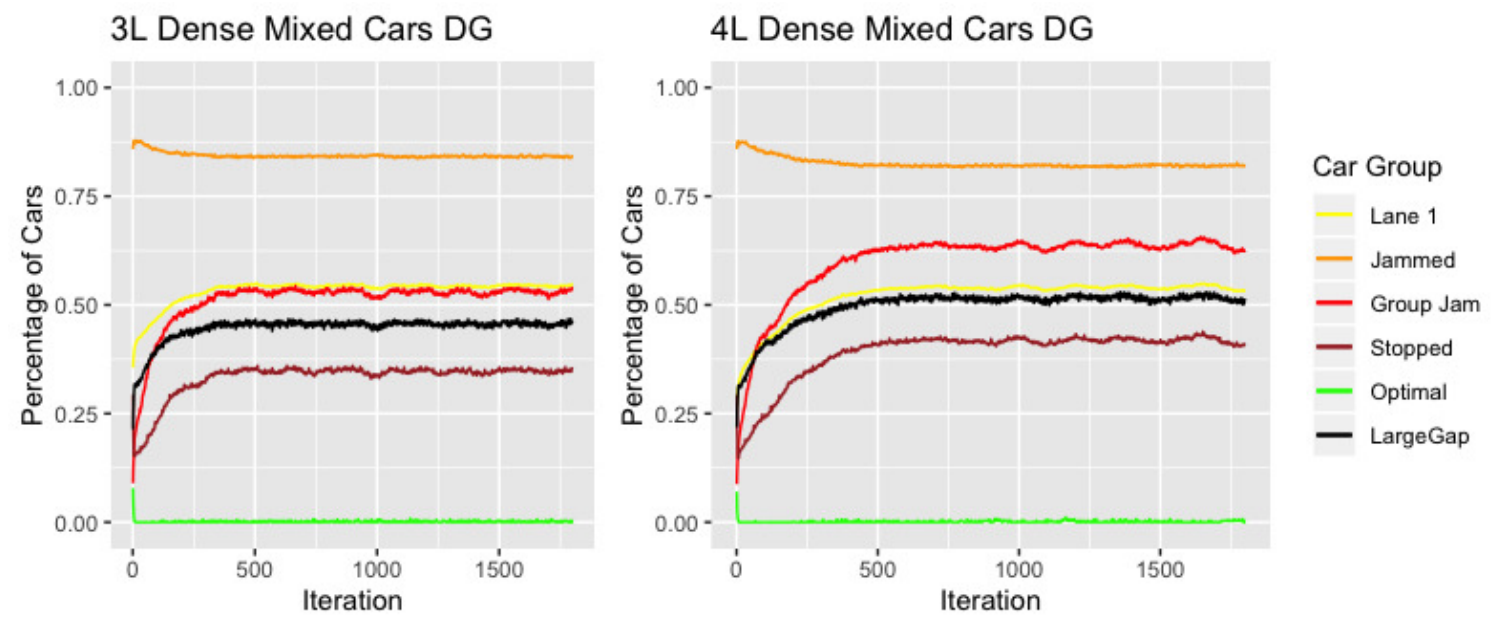

Figure B.3: Dynamics-Graph for the 3 lane (left) and 4 lane (right) Dense Open Road Experiments 

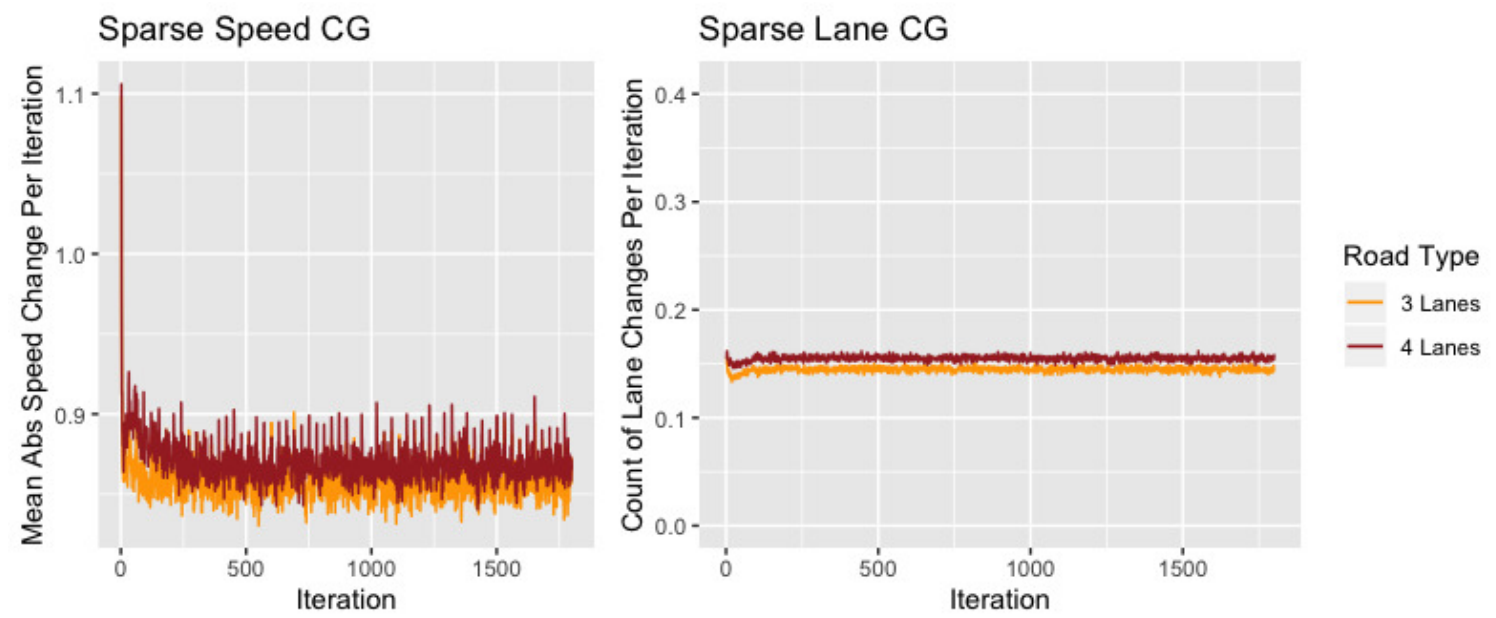

Figure B.4: Change-graph for the 3 lane and 4 lane Dense Open Road Experiments

The associated change-graph for the dense roads, in Figure B.4, shows how in both cases, the number of cars changing lanes was relatively and constantly low, while the number of speed changes per iteration was both noisy and quite high.

\section{B.2 Experiments Involving Changing Densities}

This section focuses on experiments where the density target varied throughout the experiment. Even though cars made decisions based on local information, and density is a global factor, changing the density target did, indeed, create trends in the behaviour of all cars locally as well. These experiments compared roads with different numbers of lanes; the first experiment increased the number of cars by a constant amount regardless of the number of lanes on the road, while the second experiment increased the number of cars by a constant density, which changed the target number of cars depending on the number of lanes. 

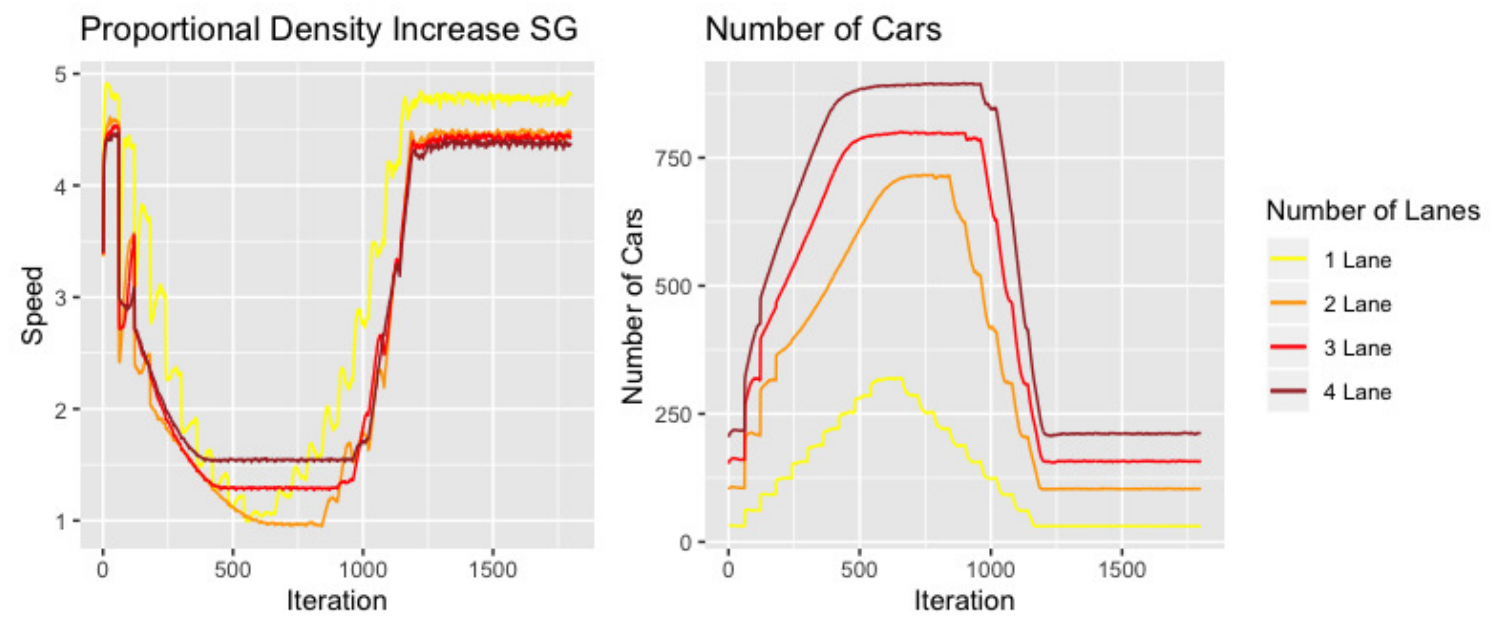

Figure B.5: Comparison of constant density changes for 1 lane, 2 lane, 3 lane, and 4 lane roads. All experiments started with 50 cars, and after every 60 iterations, the density target was increased by 50 cars for 10 iterations, and then decreased by 50 cars again for 10 iterations, finishing with the final 10 iterations again at a target of 50 cars. The speed graph is given on the left, and graph plotting the number of cars is on the right.
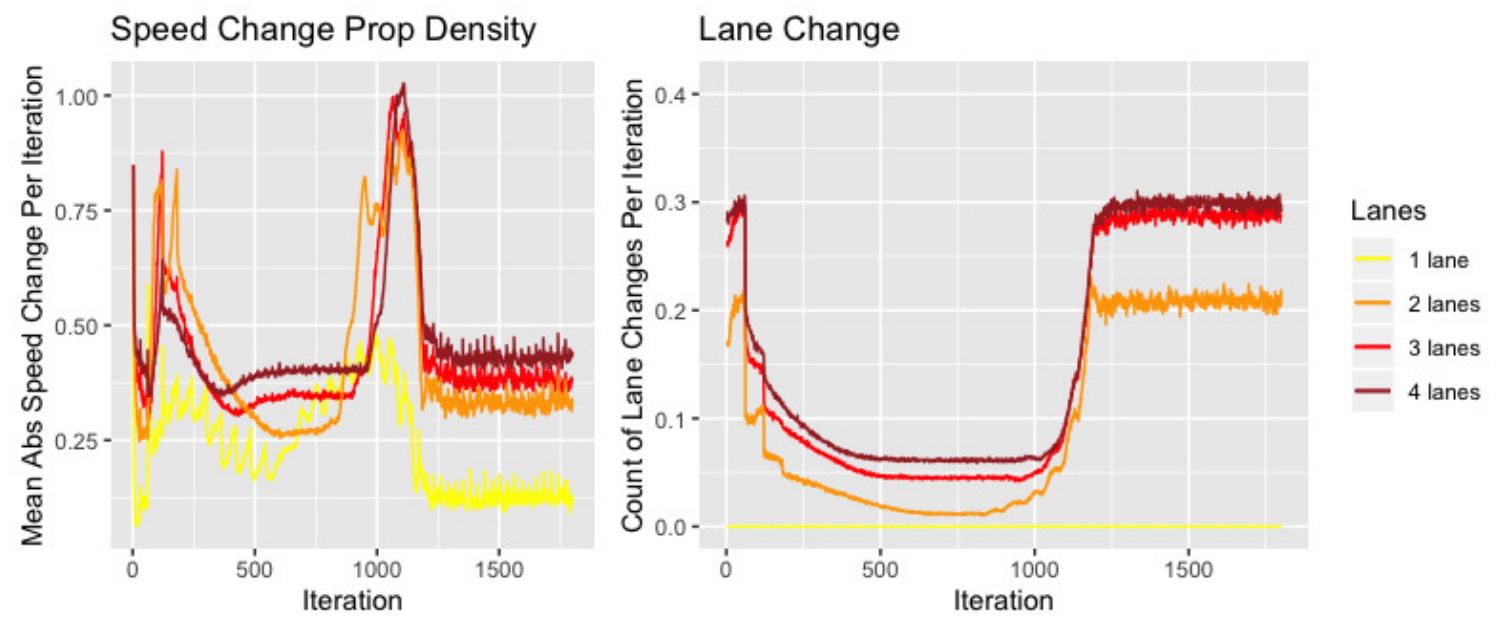

Figure B.6: The change-graph for the constant density changes across multiple lanes. 
APPENDIX B. APPENDIX B

\section{B.2.1 Changing Density Targets by a Constant Number of Cars}

The first experiment involved changing density targets by constant intervals. In this experiment, all roads were initialized with 50 cars, regardless of the number of lanes, and then the density target was increased by 50 cars every 60 iterations for a total of 600 iterations. Subsequently, this process was reversed by subtracting 50 cars from the density target for the next 600 iterations, with the final 600 iterations spent with a 50 car target. These roads contained the forced slowdown for 25 cells in the first lane, except for in the single-lane case, involved perturbations every 30 iterations, and also had an expected exit time of 200 iterations for each car.

As expected, with more lanes, because the number of cars added was constant, the impact on speed was not as noticeable, as shown in Figure B.5. It is extremely surprising, in fact, that the speed of the four lane road dropped linearly, because at peak, the target density of the road was 0.125 , which technically means that all cars could have remained at close to optimal speeds the whole time! The speed in the 4 lane case also recovered linearly, compared to the single lane case which gradually decreased in average speed and then much more suddenly increases.

As seen in the Speed-Graph in Figure B.6, whenever cars were added, the overall speed of the road decreased sharply but then increased again until the target number of cars on the road was increased again. Furthermore, the number of lane changes was consistently higher for a higher number of lanes, which is analogous with the sparse roads scenario analyzed above.

\section{B.2.2 Changing Density Targets by a Proportionally by Den- sity}

The final experiment involved proportionally changing density targets, accomplished by starting each road at a 0.05 density target, and then increasing the density target by 0.05 every 60 iterations for 600 iterations, and thereafter reversing it for 600 more iterations, and then maintaining the 0.05 density target until the end. In this case, the 0.05 density target referred to 50 cars in a one lane road, 100 in a two lane road, 

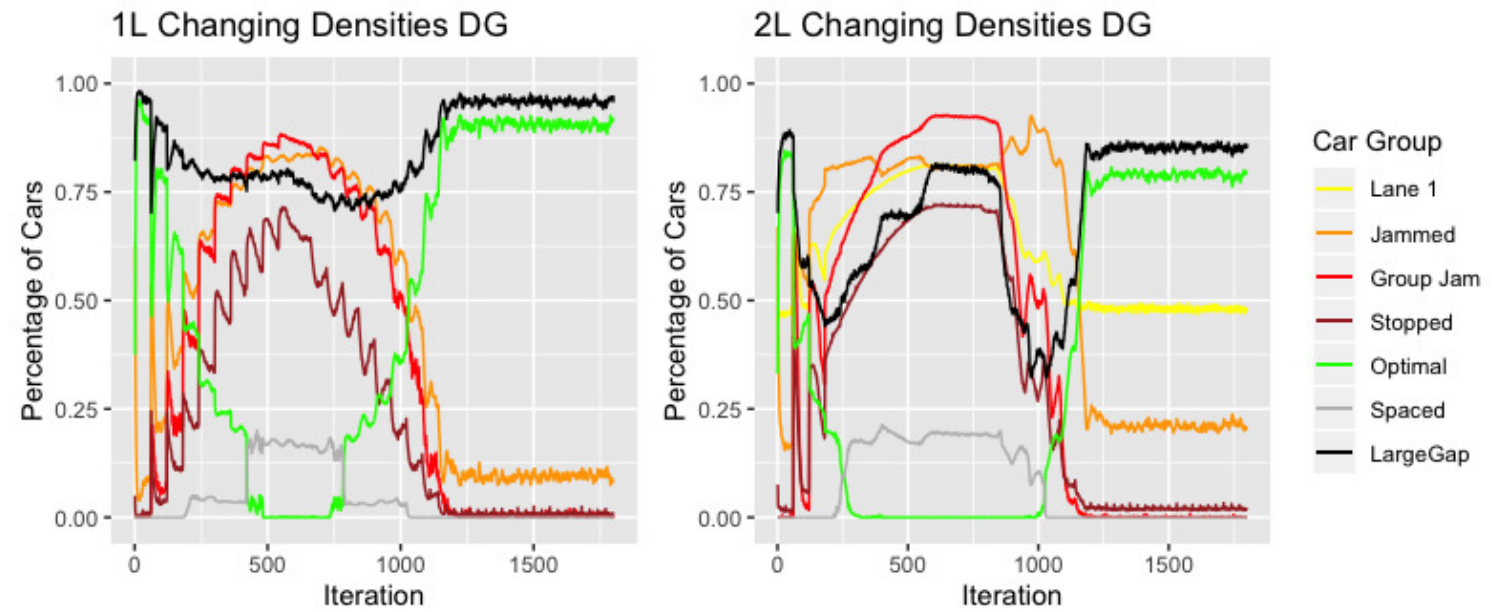

Figure B.7: Comparison of proportional density changes for 1 lane, 2 lane, 3 lane, and 4 lane roads. All experiments started with a density of 0.05 , and on every 60 iterations, the density target was increased by 0.05 for 10 iterations, and then decreased by 0.05 again for 10 iterations, finishing with the final 10 iterations again at the initial density of 0.05 . The speed graph is given on the left, and graph plotting the number of cars is on the right.

3L Changing Densities DG

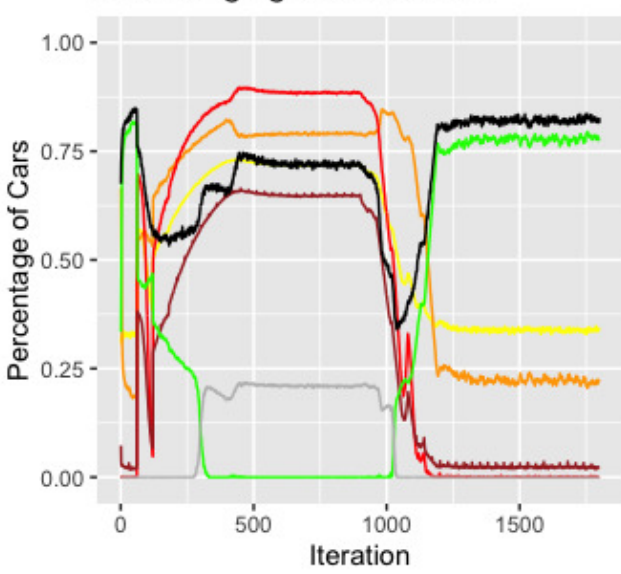

4L Changing Densities DG

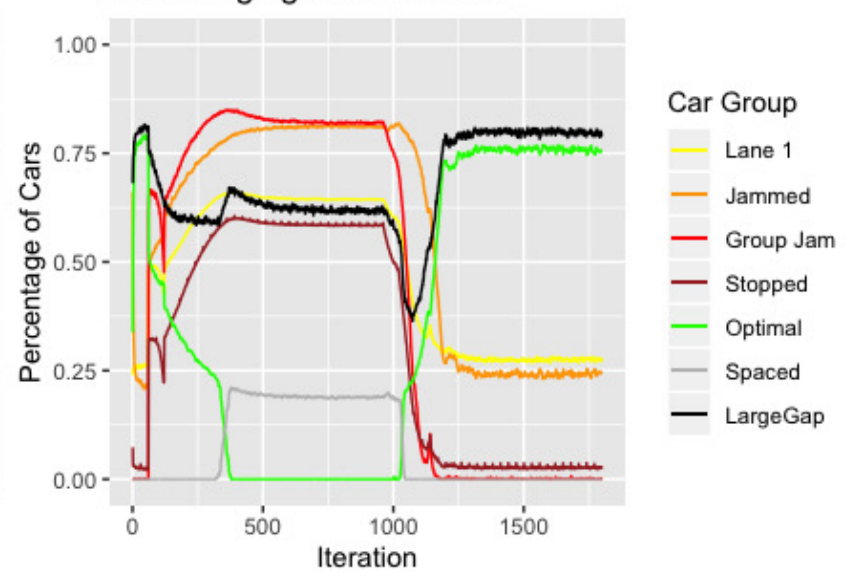

Figure B.8: The change-graph for the proportional density changes across multiple lanes. 

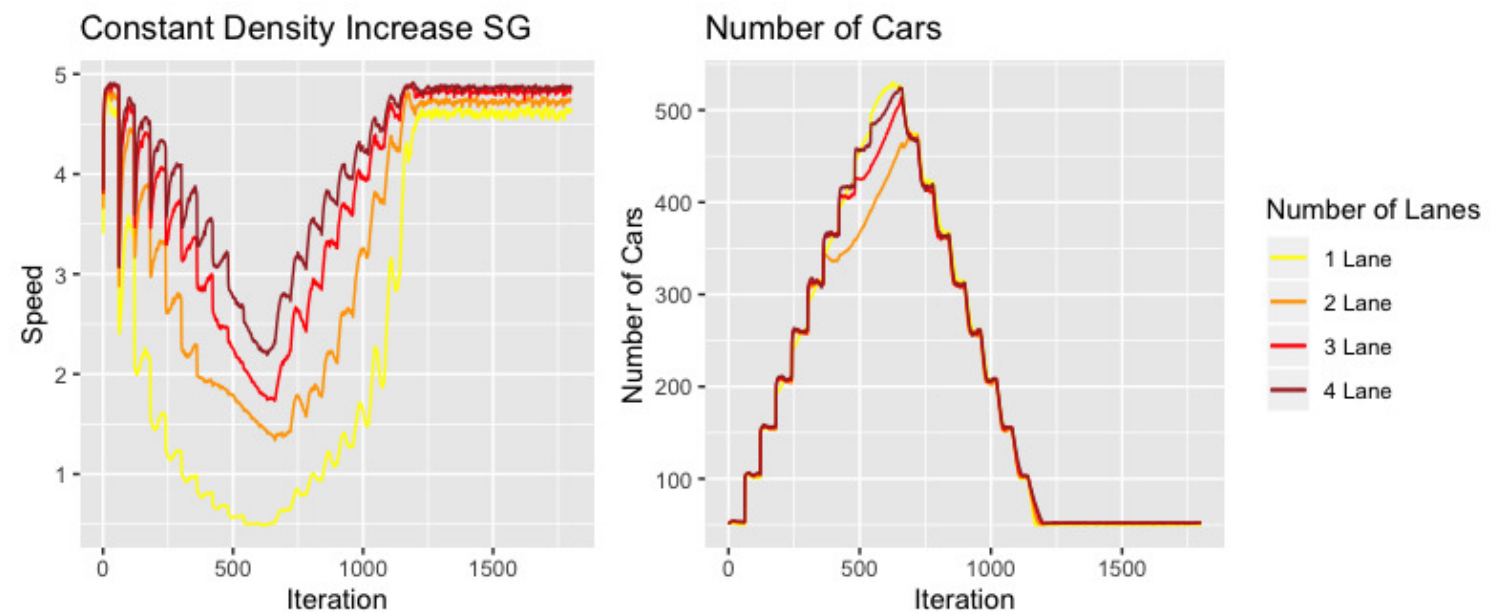

Figure B.9: The dynamics-graph for the 1 lane (left) and 2 lane (right) proportional density change experiments.
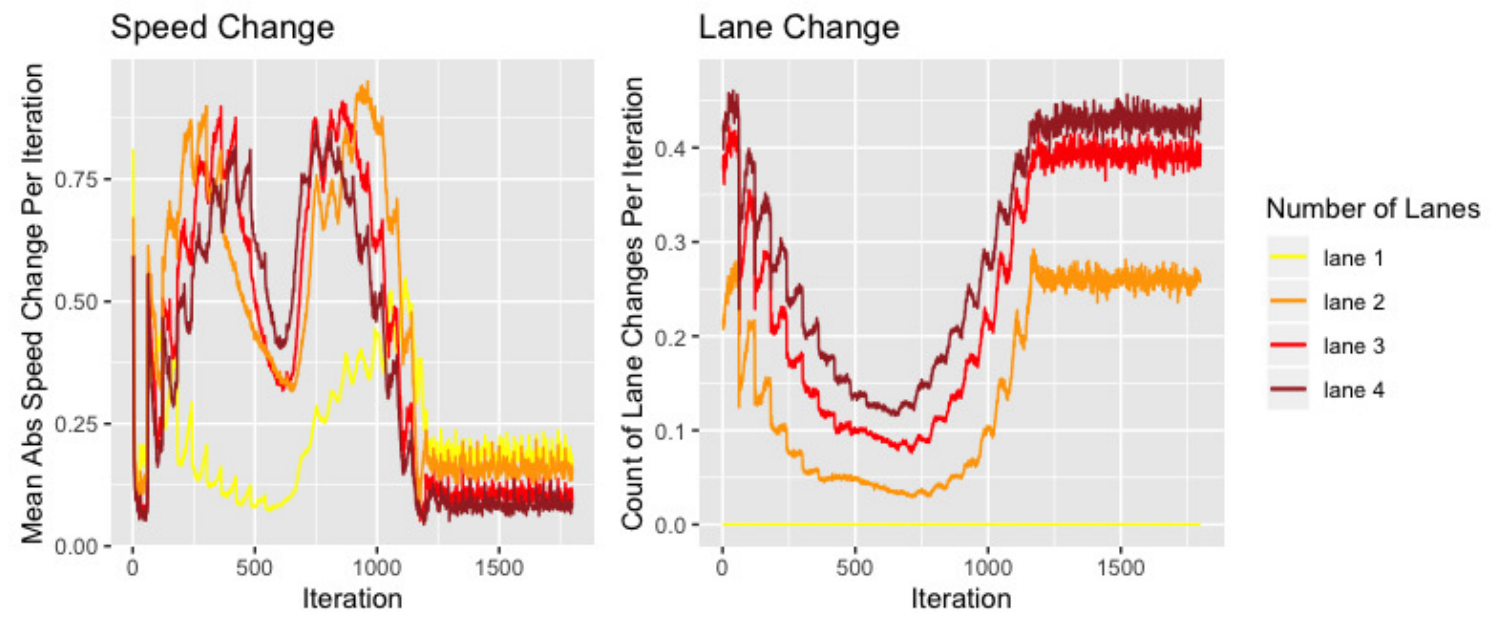

Figure B.10: The dynamics-graph for the 3 lane (left) and 4 lane (right) proportional density change experiments. 
and so on. Furthermore, in this experiment, the one lane road had a 200 iteration expected exit, while the two, three, and four lane roads had a 400, 600, and 800 iteration expected exit time, respectively, for each car.

The Speed Graph, given in B.7, shows how when the road became more dense, the speed quickly decreased almost to an average speed of 0.5 for the single lane roads, and then increased again, as expected. As can be seen, the total number of cars in the 4 lane case never attained the actual peak target of 2000, because there were not enough spaces for cars to enter the road. However, the proportional impact of changing densities for multi-lane cases is seen, and clearly, the roads were better at accelerating quickly once space was created, compared to the cars where one had to adjust after more cars were added.

The Change Graph and Dynamics Graphs are given as well for all lane types, in Figure B.8, Figure B.9, and Figure B.10.

The biggest surprise is that in the end, though the road finished with the same density as the first single lane experiment; because cars are entering and exiting, the average speed was never able to reach the maximum. This is intuitively appealing! The Change Graph shows that within each change in densities, as the road became more dense, the initial speed dropped, but then quickly increased again when the cars were removed. When the road density was decreased, the speed of cars quickly rose and then stabilized as cars went from suddenly having a larger Front Gap, to once again entering stop-and-go waves.

In the Dynamics Graph, given in Figure B.10, the percentage of stopped cars increased rapidly as the density increased, and stayed relatively high until the density changes stopped. This appeared to cause more grouped jams compared to the percentage of stopped cars. Furthermore, when the density increased, the percentage of spaced cars actually increased, as did the number of cars with a large Front Gap, even though the number of optimal cars dropped to 0. 


\section{B.3 Conclusions}

It does appear generally that two lane roads had the toughest time adjusting to higher densities, compared with three and four lane roads. Also, in simulations where the density target was being increased, higher-lane roads reacted proportionally by slowing down more than was relatively "required". 